\title{
FREE-ENERGY-DISSIPATIVE SCHEMES FOR THE OLDROYD-B MODEL
}

\author{
Sébastien Boyaval ${ }^{1,2}$, Tony Lelièvre $^{1,2}$ and Claude Mangoubi ${ }^{1,2,3}$
}

\begin{abstract}
In this article, we analyze the stability of various numerical schemes for differential models of viscoelastic fluids. More precisely, we consider the prototypical Oldroyd-B model, for which a free energy dissipation holds, and we show under which assumptions such a dissipation is also satisfied for the numerical scheme. Among the numerical schemes we analyze, we consider some discretizations based on the log-formulation of the Oldroyd-B system proposed by Fattal and Kupferman in [J. NonNewtonian Fluid Mech. 123 (2004) 281-285], for which solutions in some benchmark problems have been obtained beyond the limiting Weissenberg numbers for the standard scheme (see [Hulsen et al. J. Non-Newtonian Fluid Mech. 127 (2005) 27-39]). Our analysis gives some tracks to understand these numerical observations.
\end{abstract}

Mathematics Subject Classification. 65M12, 76M10, 35B45, 76A10, 35B35.

Received January 15, 2008. Revised September 16, 2008.

Published online April 8, 2009.

\section{INTRODUCTION}

\subsection{The stability issue in numerical schemes for viscoelastic fluids}

An abundant literature has been discussing for over twenty years the stability of numerical schemes for discretizing equations modelling viscoelastic fluids (see $[16,27,28,32]$ for a small sample). Indeed, most numerical schemes for macroscopic constitutive equations are known to suffer from instabilities in some benchmark problems, especially when a parameter, the Weissenberg number, increases.

Many possible reasons of that so-called high Weissenberg number problem (HWNP) have been identified $[15,26,31,42]$. However, these results have not led yet to a complete understanding of the numerical instabilities [28], despite some progress [15,23]. Roughly speaking, we can distinguish between three possible causes of the HWNP:

(1) Absence of stationary state: In many situations (flow past a cylinder, 4:1 contraction), the existence of a stationary state for viscoelastic models is still under investigation. It may happen that the nonconvergence of the numerical scheme is simply due to the fact that, for the model under consideration, there exists no stationary state while the numerical scheme implicitly assumes such a stationary state.

Keywords and phrases. Viscoelastic fluids, Weissenberg number, stability, entropy, finite elements methods, discontinuous Galerkin method, characteristic method.

1 CERMICS, École Nationale des Ponts et Chaussées (ParisTech/Université Paris-Est), 6 \& 8 avenue Blaise Pascal, Cité Descartes, 77455 Marne-la-Vallée Cedex 2, France. boyaval@cermics.enpc.fr; lelievre@cermics.enpc.fr;

mangoubi@cermics.enpc.fr

2 MICMAC team-project, INRIA, Domaine de Voluceau, BP. 105, Rocquencourt, 78153 Le Chesnay Cedex, France.

3 Institute of Mathematics, The Hebrew University, Jerusalem 91904, Israel. 
(2) Instabilities for the exact solution: More generally, the instabilities observed for the numerical scheme may originate at the continuous level, for the model under consideration, if the solution to the problem indeed blows up in finite time, or if it is not sufficiently regular to be well approximated in the discretization spaces. Such situations are known to occur for the Oldroyd-B model in extensional flows, for example (see $[2,41,46])$.

(3) Bad numerical scheme: It may also happen that the problem at the continuous level indeed admits a regular solution, and the instabilities are only due to the discretization method.

In this paper, we focus on the third origin of instabilities, and we propose a criterion to test the stability of numerical schemes. More precisely, we look under which conditions a numerical scheme does not bring spurious free energy in the system. We concentrate on the Oldroyd-B model, for which a free energy dissipation is known to hold at the continuous level (see Thm. 2.2 below and [20]) and we try to obtain a similar dissipation at the discrete level. It is indeed particularly important that no spurious free energy is brought to the system in long-time computations, since they are often used as a way to obtain the stationary state.

The Oldroyd-B system of equations is definitely not a good physical model for dilute polymer fluids. In particular, it can be derived from a kinetic theory, with dumbbells modeling polymer molecules that are unphysically assumed to be infinitely extensible (and this indeed seems to be the cause of some instabilities for the flow of an Oldroyd-B fluid past a cylinder, see $[2,41,46])$. But from the mathematical viewpoint, it is nevertheless a good first step into the study of macroscopic constitutive equations for viscoelastic fluids. Indeed, it already contains mathematical difficulties common to most of the viscoelastic models, while its strict equivalence with a kinetic model allows for a deep understanding of this set of equations. Let us also emphasize that the free energy dissipation we use and the numerical schemes we consider are not restricted to the Oldroyd-B model: they can be generalized to many other models (like FENE-P for instance, see [20]), so that we believe that our analysis can be used as a guideline to derive "good" numerical schemes for many macroscopic models for viscoelastic fluids. In summary, our aim here is not to discuss the HWNP but to propose a new criterion to assess the stability of numerical schemes for viscoelastic flows.

\subsection{Mathematical setting of the problem}

We consider the Oldroyd-B model for dilute polymeric fluids in $d$-dimensional flows $(d=2,3)$. Confined to an open bounded domain $\mathcal{D} \subset \mathbb{R}^{d}$, the fluid is governed by the following nondimensionalized system of equations:

$$
\left\{\begin{aligned}
& \operatorname{Re}\left(\frac{\partial \boldsymbol{u}}{\partial t}+\boldsymbol{u} \cdot \boldsymbol{\nabla} \boldsymbol{u}\right)=-\boldsymbol{\nabla} p+(1-\varepsilon) \Delta \boldsymbol{u}+\operatorname{div} \boldsymbol{\tau} \\
& \operatorname{div} \boldsymbol{u}=0 \\
& \frac{\partial \boldsymbol{\tau}}{\partial t}+(\boldsymbol{u} \cdot \boldsymbol{\nabla}) \boldsymbol{\tau}=(\boldsymbol{\nabla} \boldsymbol{u}) \boldsymbol{\tau}+\boldsymbol{\tau}(\boldsymbol{\nabla} \boldsymbol{u})^{T}-\frac{1}{\mathrm{Wi}} \boldsymbol{\tau}+\frac{\varepsilon}{\mathrm{Wi}}\left[\boldsymbol{\nabla} \boldsymbol{u}+\boldsymbol{\nabla} \boldsymbol{u}^{T}\right]
\end{aligned}\right.
$$

where $\boldsymbol{u}:(t, \boldsymbol{x}) \in[0, T) \times \mathcal{D} \rightarrow \boldsymbol{u}(t, \boldsymbol{x}) \in \mathbb{R}^{d}$ is the velocity of the fluid, $p:(t, \boldsymbol{x}) \in[0, T) \times \mathcal{D} \rightarrow p(t, \boldsymbol{x}) \in \mathbb{R}$ is the pressure and $\boldsymbol{\tau}:(t, \boldsymbol{x}) \in[0, T) \times \mathcal{D} \rightarrow \boldsymbol{\tau}(t, \boldsymbol{x}) \in \mathbb{R}^{d \times d}$ is the extra-stress tensor. The matrix $\boldsymbol{\nabla} \boldsymbol{u}$ is the $d \times d$ matrix with components $\left(\frac{\partial \boldsymbol{u}_{i}}{\partial \boldsymbol{x}_{j}}\right)_{i, j}$. The following parameters are dimensionless: the Reynolds number Re $\in \mathbb{R}_{+}$ (where $\mathbb{R}_{+}=[0,+\infty)$ ), the Weissenberg number Wi $\in \mathbb{R}_{+}^{*}\left(\right.$ where $\left.\mathbb{R}_{+}^{*}=(0,+\infty)\right)$ and the elastic viscosity to total viscosity fraction $\varepsilon \in(0,1)$.

In what follows, we assume for the sake of simplicity that the system (1.1) is supplied with homogeneous Dirichlet boundary conditions for the velocity $\boldsymbol{u}$ :

$$
\boldsymbol{u}=0 \text { on } \partial \mathcal{D}
$$

Therefore, we study the energy dissipation of the equations (1.1) as time goes, that is, the way $(\boldsymbol{u}, \boldsymbol{\tau})$ converges to the stationary state $(0,0)$ (equilibrium) in the long-time limit $t \rightarrow \infty$. Let us mention that it is possible to extend the analysis to non-zero boundary conditions (or more generally non-zero forcing) in the following way: 
it can be shown (see [24]) that if the stationary velocity is not too large, then exponential convergence to the stationary state is achieved, at the continuous level. The schemes we propose are likely to exhibit similar behaviour, but we have not checked all the details for such a situation.

Local-in-time existence results for the above problem have been proved in the bounded domain $[0, T) \times \mathcal{D}$ when the system is supplied with sufficiently smooth initial conditions $\boldsymbol{u}(t=0)$ and $\boldsymbol{\tau}(t=0)$ (see [17,19] for instance). Moreover, global-in-time smooth solutions of the system (1.1) are known to converge exponentially fast to equilibrium in the sense defined in [24]. Let us also mention the work of Lin et al. [34] where, for Oldroyd-like models, local-in-time existence and uniqueness results are proven, but also global-in-time existence and uniqueness results for small data. Notice that more general global-in-time results have been collected only for a mollified version of the Oldroyd-B system (1.1) (see [5]), for another system close to (1.1) namely the corotational Oldroyd-B system (see [35]), or in the form of a Beale-Kato-Majda criterion when $\mathcal{D}=\mathbb{R}^{3}$ (see [29]). Even though the question of the global-in-time existence for some solutions of the Oldroyd-B system (1.1) is still out-of-reach, it is possible to analyze global-in-time existence for solutions to discretizations of that system. This will be one of the output of this article.

\subsection{Outline of the paper and results}

We will show that it is possible to build numerical (time and space discretizing) schemes for the Oldroyd-B system (1.1)-(1.2) such that solutions to those discretizations satisfy a free energy estimate similar to that established in $[20,24]$ for smooth solutions to the continuous equations. Our approach bears similarity with [36], where the authors also derive a discretization that preserves an energy estimate satisfied at the continuous level, and with [32], where another discretization is proposed for the same energy estimate as in [36]. Yet, unlike the estimates in [32,36], our estimate, the so-called free energy estimate derived in [20,24], ensures (free) energy dissipation and exponential convergence of the solution to equilibrium. In particular, the long-time stability of solutions is ensured. As mentioned above, long-time computations are indeed often used to obtain a stationary state, so that such a property may be seen as an interesting feature of a numerical scheme.

We also analyze discretizations of the log-formulation presented in $[14,15]$, where the authors suggest to rewrite the set of equations (1.1) after mapping the (symmetric positive definite) conformation tensor:

$$
\boldsymbol{\sigma}=\boldsymbol{I}+\frac{\mathrm{Wi}}{\varepsilon} \boldsymbol{\tau}
$$

to its matrix logarithm:

$$
\psi=\ln \sigma
$$

In the following, we assume that:

$$
\boldsymbol{\sigma}(t=0) \text { is symmetric positive definite, }
$$

and it can be shown that this property is propagated in time (see Lem. 2.1 below), so that $\boldsymbol{\psi}$ is indeed well defined. The log-formulation ensures, by construction, that the conformation tensor always remains symmetric positive definite, even after discretization. This is not only an important physical characteristic of the Oldroyd-B model but also an essential feature in the free energy estimates derived beneath. Besides, in some benchmark problems $[15,23,30]$, discretizations of the log-formulation have indeed been reported to yield solutions beyond the limiting Weissenberg number for standard discretizations of the usual formulation (for the Oldroyd-B and the Giesekus models). It is thus interesting to investigate whether the numerical success of this log-formulation may be related to a free energy dissipation property.

The main outputs of this work are:

(i) One crucial feature of the numerical scheme to obtain free energy estimates is the appropriate discretization of the advection term $(\boldsymbol{u} \cdot \boldsymbol{\nabla}) \boldsymbol{\tau}$ (or $(\boldsymbol{u} \cdot \boldsymbol{\nabla}) \boldsymbol{\psi}$ in the log-formulation) in the equation on the extra-stress tensor. We will analyze below two types of discretization: the characteristic method, and the discontinuous Galerkin method (see Sect. 4, and Appendix D for higher-order schemes). 
(ii) To obtain free energy estimates, we will need the extra-stress tensor to be discretized in a (elementwise) discontinuous finite element space (Sect. 4 and Appendix D).

(iii) The existence of a solution to the numerical schemes that satisfies a free energy estimate will be proved whatever the time step for the log-formulation in terms of $\boldsymbol{\psi}$, while it will be shown under a CFL-like condition for the usual formulation in terms of $\boldsymbol{\tau}$ (see Sect. 5). Moreover, any solution to the logformulation satisfies the free energy estimate (which is not the case for the usual formulation in terms of $\boldsymbol{\tau}$. This may be related to the fact that the log-formulation has been reported to be more stable than the formulation in terms of $\boldsymbol{\tau}$ (see [23]).

We would like to mention the work in preparation [4] where the existence of a solution to a numerical scheme which satisfies a free energy estimate is also obtained whatever the time step for the usual formulation of the Oldroyd-B model in terms of $\boldsymbol{\sigma}$, but only as the limit of a subsequence of regularized discretizations. This means that, in the case where the CFL condition is not fulfilled hence uniqueness not ensured, there may be many solutions to our numerical schemes for the usual formulation of the Oldroyd-B model in terms of $\boldsymbol{\sigma}$, one of which is actually shown to satisfy a free energy estimate; on the contrary, every solution to our numerical schemes for the log-formulation necessarily satisfies a free energy estimate. Moreover, it is shown in this work [4] that, using a particular discretization of the advection term, it is possible to use continuous finite element spaces to obtain a discrete analogue of the free energy bound for a regularized Oldroyd-B model. In addition, subsequence convergence, as the mesh parameters tend to zero, of such a scheme is proved, which yields existence of global-in-time solutions to this modified Oldroyd-B system.

Notice that we here concentrate on stability issues. All the schemes we analyze are of course consistent, but we do not study the order of consistency of these schemes, neither the convergence.

Let us now make precise how the paper is organized. In Section 2, we formally derive the free energy estimates for the Oldroyd-B set of equations and for its logarithm formulation, in the spirit of [20]. Then, Section 3 is devoted to the presentation of a finite element scheme (using piecewise constant approximations of the conformation tensor and its log-formulation, and Scott-Vogelius finite elements for the velocity and pressure), that is shown to satisfy a discrete free energy estimate in Section 4. Some variants of this discretization are also studied, still for piecewise constant stress tensor, and a summary of the requirements on the discretizations to satisfy a free energy estimate is provided in Tables 1 and 2 (we show in Appendix D how to use an interpolation operator so as to adapt the previous results to piecewise linear approximations of the stress tensor). Finally, in Section 5, we show how the previous stability results can be used to prove long-time existence results for the discrete solutions. Some numerical studies are needed to illustrate this numerical analysis, and this is a work in progress.

\subsection{Notation and auxiliary results}

In the following, we will make use of the usual notation: $L^{2}(\mathcal{D})=\left\{f: \mathcal{D} \rightarrow \mathbb{R}, \int_{\mathcal{D}}|f|^{2}<\infty\right\}, H^{1}(\mathcal{D})=$ $\left\{f: \mathcal{D} \rightarrow \mathbb{R}, \int_{\mathcal{D}}|f|^{2}+|\nabla f|^{2}<\infty\right\}, H^{2}(\mathcal{D})=\left\{f: \mathcal{D} \rightarrow \mathbb{R}, \int_{\mathcal{D}}|f|^{2}+|\nabla f|^{2}+\left|\nabla^{2} f\right|^{2}<\infty\right\}, C([0, T))$ for continuous functions on $[0, T)$ and $C^{1}([0, T))$ for continuously differentiable functions on $[0, T)$.

We will denote by $\boldsymbol{\tau}: \boldsymbol{\sigma}$ the double contraction between rank-two tensors (matrices) $\boldsymbol{\tau}, \boldsymbol{\sigma} \in \mathbb{R}^{d \times d}$ :

$$
\boldsymbol{\tau}: \boldsymbol{\sigma}=\operatorname{tr}\left(\boldsymbol{\tau} \boldsymbol{\sigma}^{T}\right)=\operatorname{tr}\left(\boldsymbol{\tau}^{T} \boldsymbol{\sigma}\right)=\sum_{1 \leq i, j \leq d} \boldsymbol{\tau}_{i j} \boldsymbol{\sigma}_{i j}
$$

Notice that if $\boldsymbol{\tau}$ is antisymmetric and $\boldsymbol{\sigma}$ symmetric, $\boldsymbol{\tau}: \boldsymbol{\sigma}=0$.

The logarithm of a positive definite diagonal matrix is a diagonal matrix with, on its diagonal, the logarithm of each entry. We define the logarithm of any symmetric positive definite matrix $\boldsymbol{\sigma}$ using a diagonal decomposition $\boldsymbol{\sigma}=R^{T} \Lambda R$ of $\boldsymbol{\sigma}$ with $R$ an orthogonal matrix and $\Lambda$ a positive definite diagonal matrix:

$$
\ln \sigma=R^{T}(\ln \Lambda) R
$$


Although the diagonal decomposition of $\boldsymbol{\sigma}$ is not unique, (1.5) uniquely defines $\ln \boldsymbol{\sigma}$. The matrix logarithm bijectively maps the set of symmetric positive definite matrices with real entries $\mathcal{S}_{+}^{*}\left(\mathbb{R}^{d \times d}\right)$ to the vector subspace $\mathcal{S}\left(\mathbb{R}^{d \times d}\right)$ of symmetric real matrices, where it is exactly the inverse function of the matrix exponential.

We will make use of the following simple algebraic formulae, which are proved in Appendices A.1 and A.2.

Lemma 1.1. Let $\boldsymbol{\sigma}$ and $\boldsymbol{\tau}$ be two symmetric positive definite matrices. We have:

$$
\begin{gathered}
\operatorname{tr} \ln \boldsymbol{\sigma}=\ln \operatorname{det} \boldsymbol{\sigma} \\
\boldsymbol{\sigma}-\ln \boldsymbol{\sigma}-\boldsymbol{I} \text { is symmetric positive semidefinite and thus } \operatorname{tr}(\boldsymbol{\sigma}-\ln \boldsymbol{\sigma}-\boldsymbol{I}) \geq 0, \\
\boldsymbol{\sigma}+\boldsymbol{\sigma}^{-1}-2 \boldsymbol{I} \text { is symmetric positive semidefinite and thus } \operatorname{tr}\left(\boldsymbol{\sigma}+\boldsymbol{\sigma}^{-1}-2 \boldsymbol{I}\right) \geq 0, \\
\operatorname{tr}(\boldsymbol{\sigma} \boldsymbol{\tau})=\operatorname{tr}(\boldsymbol{\tau} \boldsymbol{\sigma}) \geq 0 \\
\operatorname{tr}\left((\boldsymbol{\sigma}-\boldsymbol{\tau}) \boldsymbol{\tau}^{-1}\right)=\operatorname{tr}\left(\boldsymbol{\sigma} \boldsymbol{\tau}^{-1}-\boldsymbol{I}\right) \geq \ln \operatorname{det}\left(\boldsymbol{\sigma} \boldsymbol{\tau}^{-1}\right)=\operatorname{tr}(\ln \boldsymbol{\sigma}-\ln \boldsymbol{\tau}), \\
\operatorname{tr}((\ln \boldsymbol{\sigma}-\ln \boldsymbol{\tau}) \boldsymbol{\sigma}) \geq \operatorname{tr}(\boldsymbol{\sigma}-\boldsymbol{\tau})
\end{gathered}
$$

We will also use the Jacobi's formulae:

Lemma 1.2. For any symmetric positive definite matrix $\boldsymbol{\sigma}(t) \in\left(C^{1}([0, T))\right)^{\frac{d(d+1)}{2}}$, we have $\forall t \in[0, T)$ :

$$
\begin{gathered}
\left(\frac{\mathrm{d}}{\mathrm{d} t} \boldsymbol{\sigma}\right): \boldsymbol{\sigma}^{-1}=\operatorname{tr}\left(\boldsymbol{\sigma}^{-1} \frac{\mathrm{d}}{\mathrm{d} t} \boldsymbol{\sigma}\right)=\frac{\mathrm{d}}{\mathrm{d} t} \operatorname{tr}(\ln \boldsymbol{\sigma}), \\
\left(\frac{\mathrm{d}}{\mathrm{d} t} \ln \boldsymbol{\sigma}\right): \boldsymbol{\sigma}=\operatorname{tr}\left(\boldsymbol{\sigma} \frac{\mathrm{d}}{\mathrm{d} t} \ln \boldsymbol{\sigma}\right)=\frac{\mathrm{d}}{\mathrm{d} t} \operatorname{tr} \boldsymbol{\sigma} .
\end{gathered}
$$

\section{Formal free energy estimates at the continuous LeVel}

We are going to derive free energy estimates for two formulations of the Oldroyd-B system in Theorems 2.2 and 2.4. An important corollary to these theorems is the exponential convergence of the solutions to equilibrium in the long-time limit. Throughout this section, we assume that $(\boldsymbol{u}, p, \boldsymbol{\tau})$ is a sufficiently smooth solution of problem (1.1) so that all the subsequent computations are valid. For example, the following regularity is sufficient:

$$
(\boldsymbol{u}, p, \boldsymbol{\tau}) \in\left(C^{1}\left([0, T), H^{2}(\mathcal{D}) \cap C^{0,1}(\mathcal{D})\right)\right)^{d} \times\left(C^{0}\left([0, T), H^{1}(\mathcal{D})\right)\right) \times\left(C^{1}\left([0, T), C^{1}(\mathcal{D})\right)\right)^{d \times d},
$$

where we denote, for instance by $\left(C^{1}(\mathcal{D})\right)^{d}$ a vector field of dimension $d$ with $C^{1}(\mathcal{D})$ components.

\subsection{Free energy estimate for the Oldroyd-B system}

\subsubsection{Conformation-tensor formulation of the Oldroyd-B system}

Recall that the conformation tensor $\boldsymbol{\sigma}$ is defined from the extra-stress tensor $\boldsymbol{\tau}$ through the following bijective mapping:

$$
\boldsymbol{\tau}=\frac{\varepsilon}{\mathrm{Wi}}(\boldsymbol{\sigma}-\boldsymbol{I})
$$

With this mapping, it is straightforward to bijectively map the solutions of system (1.1) with those of the following system:

$$
\left\{\begin{aligned}
& \operatorname{Re}\left(\frac{\partial \boldsymbol{u}}{\partial t}+\boldsymbol{u} \cdot \boldsymbol{\nabla} \boldsymbol{u}\right)=-\boldsymbol{\nabla} p+(1-\varepsilon) \Delta \boldsymbol{u}+\frac{\varepsilon}{\mathrm{Wi}} \operatorname{div} \boldsymbol{\sigma} \operatorname{div} \boldsymbol{u}=0 \\
& \frac{\partial \boldsymbol{\sigma}}{\partial t}+(\boldsymbol{u} \cdot \boldsymbol{\nabla}) \boldsymbol{\sigma}=(\boldsymbol{\nabla} \boldsymbol{u}) \boldsymbol{\sigma}+\boldsymbol{\sigma}(\boldsymbol{\nabla} \boldsymbol{u})^{T}-\frac{1}{\mathrm{Wi}}(\boldsymbol{\sigma}-\boldsymbol{I}) .
\end{aligned}\right.
$$


Notice that with such an affine mapping, the solution $\boldsymbol{\sigma}$ to system (2.2) has the same regularity as $\boldsymbol{\tau}$ solution to system (1.1), which is that assumed in (2.1) for the following manipulations.

\subsubsection{A free energy estimate}

Let us first recall a free energy estimate derived in [20,24]. The free energy of the fluid is defined as the sum of two terms as follows:

$$
F(\boldsymbol{u}, \boldsymbol{\sigma})=\frac{\mathrm{Re}}{2} \int_{\mathcal{D}}|\boldsymbol{u}|^{2}+\frac{\varepsilon}{2 \mathrm{Wi}} \int_{\mathcal{D}} \operatorname{tr}(\boldsymbol{\sigma}-\ln \boldsymbol{\sigma}-\boldsymbol{I}) .
$$

The kinetic term $\int_{\mathcal{D}}|\boldsymbol{u}|^{2}$ is always non negative. Besides, we have the following lemma (see Appendix B or [22] for a proof):

Lemma 2.1. Let $\boldsymbol{\sigma} \in\left(C^{1}\left([0, T), C^{1}(\mathcal{D})\right)\right)^{d \times d}$ be a smooth solution to the system (2.2). Then, if the initial condition $\boldsymbol{\sigma}(t=0)$ is symmetric positive definite (everywhere in $\mathcal{D}$ ), the solution $\boldsymbol{\sigma}(t)$ remains so at all times $t \in[0, T)$ and for all $\boldsymbol{x} \in \mathcal{D}$. In particular, the matrix $\boldsymbol{\sigma}(t)$ is invertible.

From Lemma 2.1 and the equation (1.7), the entropic term $\int_{\mathcal{D}} \operatorname{tr}(\boldsymbol{\sigma}-\ln \boldsymbol{\sigma}-\boldsymbol{I})$ is thus well defined and non negative, provided $\boldsymbol{\sigma}(t=0)$ is symmetric positive definite.

The free energy is an interesting quantity to characterize the long-time asymptotics of the solutions, and thus the stability of the system (2.2). A priori estimates using the free energy are presented in [24] for micromacro models (such as the Hookean or the FENE dumbbell models) and in [20] for macroscopic models (such as the Oldroyd-B or the FENE-P models). Similar considerations can be found in the physics literature about thermodynamic theory for viscoelastic models (see $[6,33,39,47]$ ).

For the sake of consistency, we recall results from [20]:

Theorem 2.2. Let $(\boldsymbol{u}, p, \boldsymbol{\sigma})$ be a smooth solution to system (2.2) supplied with homogeneous Dirichlet boundary conditions for $\boldsymbol{u}$, and with symmetric positive definite initial condition $\boldsymbol{\sigma}(t=0)$. The free energy satisfies:

$$
\frac{\mathrm{d}}{\mathrm{d} t} F(\boldsymbol{u}, \boldsymbol{\sigma})+(1-\varepsilon) \int_{\mathcal{D}}|\boldsymbol{\nabla} \boldsymbol{u}|^{2}+\frac{\varepsilon}{2 \mathrm{Wi}^{2}} \int_{\mathcal{D}} \operatorname{tr}\left(\boldsymbol{\sigma}+\boldsymbol{\sigma}^{-1}-2 \boldsymbol{I}\right)=0 .
$$

From this estimate, we get that $F(\boldsymbol{u}, \boldsymbol{\sigma})$ decreases exponentially fast in time to zero.

Proof of Theorem (2.2). Let $(\boldsymbol{u}, p, \boldsymbol{\sigma})$ be a smooth solution to system (2.2), with symmetric positive definite initial condition $\boldsymbol{\sigma}(t=0)$. We first compute the inner product of the Navier-Stokes equation with the velocity:

$$
\frac{\mathrm{Re}}{2} \frac{\mathrm{d}}{\mathrm{d} t} \int_{\mathcal{D}}|\boldsymbol{u}|^{2}=-(1-\varepsilon) \int_{\mathcal{D}}|\boldsymbol{\nabla} \boldsymbol{u}|^{2}-\frac{\varepsilon}{\mathrm{Wi}} \int_{\mathcal{D}} \boldsymbol{\nabla} \boldsymbol{u}: \boldsymbol{\sigma} .
$$

Then, taking the trace of the evolution equation for the conformation tensor, we obtain:

$$
\frac{\mathrm{d}}{\mathrm{d} t} \int_{\mathcal{D}} \operatorname{tr} \boldsymbol{\sigma}=2 \int_{\mathcal{D}} \boldsymbol{\nabla} \boldsymbol{u}: \boldsymbol{\sigma}-\frac{1}{\mathrm{Wi}} \int_{\mathcal{D}} \operatorname{tr}(\boldsymbol{\sigma}-\boldsymbol{I}) .
$$

Last, remember that smooth solutions $\sigma$ are invertible matrices (Lem. 2.1). Thus, contracting the evolution equation for $\sigma$ with $\sigma^{-1}$, we get:

$$
\int_{\mathcal{D}}\left(\frac{\partial}{\partial t} \boldsymbol{\sigma}+(\boldsymbol{u} \cdot \boldsymbol{\nabla}) \boldsymbol{\sigma}\right): \boldsymbol{\sigma}^{-1}=2 \int_{\mathcal{D}} \operatorname{tr}(\boldsymbol{\nabla} \boldsymbol{u})-\frac{1}{\mathrm{Wi}} \int_{\mathcal{D}} \operatorname{tr}\left(\boldsymbol{I}-\boldsymbol{\sigma}^{-1}\right) .
$$

Using (1.12) with $\boldsymbol{\sigma} \in C^{1}\left(\mathcal{D} \times[0, T), \mathcal{S}_{+}^{\star}\left(\mathbb{R}^{d \times d}\right)\right)$, we find:

$$
\int_{\mathcal{D}}\left(\frac{\partial}{\partial t} \boldsymbol{\sigma}+(\boldsymbol{u} \cdot \boldsymbol{\nabla}) \boldsymbol{\sigma}\right): \boldsymbol{\sigma}^{-1}=\int_{\mathcal{D}}\left(\frac{\partial}{\partial t}+\boldsymbol{u} \cdot \boldsymbol{\nabla}\right) \operatorname{tr}(\ln \boldsymbol{\sigma}),
$$


which can be combined with (2.7) to get, using $\operatorname{tr}(\boldsymbol{\nabla} \boldsymbol{u})=\operatorname{div} \boldsymbol{u}=0$ and $\boldsymbol{u}=0$ on $\partial \mathcal{D}$ :

$$
\frac{\mathrm{d}}{\mathrm{d} t} \int_{\mathcal{D}} \operatorname{tr} \ln \boldsymbol{\sigma}=\frac{1}{\mathrm{Wi}} \int_{\mathcal{D}} \operatorname{tr}\left(\boldsymbol{\sigma}^{-1}-\boldsymbol{I}\right) .
$$

We now combine $(2.5)+\frac{\varepsilon}{2 \mathrm{Wi}} \times(2.6)-\frac{\varepsilon}{2 \mathrm{Wi}} \times(2.8)$ to obtain $(2.4)$ :

$$
\frac{\mathrm{d}}{\mathrm{d} t}\left[\frac{\operatorname{Re}}{2} \int_{\mathcal{D}}|\boldsymbol{u}|^{2}+\frac{\varepsilon}{2 \mathrm{Wi}} \int_{\mathcal{D}} \operatorname{tr}(\boldsymbol{\sigma}-\ln \boldsymbol{\sigma}-\boldsymbol{I})\right]+(1-\varepsilon) \int_{\mathcal{D}}|\boldsymbol{\nabla} \boldsymbol{u}|^{2}+\frac{\varepsilon}{2 \mathrm{Wi}^{2}} \int_{\mathcal{D}} \operatorname{tr}\left(\boldsymbol{\sigma}+\boldsymbol{\sigma}^{-1}-2 \boldsymbol{I}\right)=0 .
$$

Since, by (1.8), we have $\operatorname{tr}\left(\boldsymbol{\sigma}+\boldsymbol{\sigma}^{-1}-2 \boldsymbol{I}\right) \geq 0$, then $F(\boldsymbol{u}, \boldsymbol{\sigma})$ decreases in time. Moreover, by (1.7) applied to $\boldsymbol{\sigma}^{-1}$, we have $\operatorname{tr}(\boldsymbol{\sigma}-\ln \boldsymbol{\sigma}-\boldsymbol{I}) \leq \operatorname{tr}\left(\boldsymbol{\sigma}+\boldsymbol{\sigma}^{-1}-2 \boldsymbol{I}\right)$. So, using the Poincaré inequality which states that there exists a constant $C_{P}$ depending only on $\mathcal{D}$ such that, for all $\boldsymbol{u} \in H_{0}^{1}(\mathcal{D})$,

$$
\int_{\mathcal{D}}|\boldsymbol{u}|^{2} \leq C_{P} \int_{\mathcal{D}}|\nabla \boldsymbol{u}|^{2}
$$

we finally obtain that $F(\boldsymbol{u}, \boldsymbol{\sigma})$ goes exponentially fast to 0 . Indeed, we have from (2.4):

$$
\frac{\mathrm{d}}{\mathrm{d} t} F(\boldsymbol{u}, \boldsymbol{\sigma}) \leq-\frac{1-\varepsilon}{C_{P}} \int_{\mathcal{D}}|\boldsymbol{u}|^{2}-\frac{\varepsilon}{2 \mathrm{Wi}^{2}} \int_{\mathcal{D}} \operatorname{tr}\left(\boldsymbol{\sigma}+\boldsymbol{\sigma}^{-1}-2 \boldsymbol{I}\right) \leq-\min \left(\frac{2(1-\varepsilon)}{\operatorname{Re} C_{P}}, \frac{1}{\mathrm{Wi}}\right) F(\boldsymbol{u}, \boldsymbol{\sigma}),
$$

so that, by a direct application of Gronwall's lemma, we get:

$$
F(\boldsymbol{u}, \boldsymbol{\sigma}) \leq F(\boldsymbol{u}(t=0), \boldsymbol{\sigma}(t=0)) \exp \left(-\min \left(\frac{2(1-\varepsilon)}{\operatorname{Re} C_{P}}, \frac{1}{\mathrm{Wi}}\right) t\right) .
$$

\subsection{Free energy estimate for the log-formulation of the Oldroyd-B system}

\subsubsection{Log-formulation of the Oldroyd-B system}

Let us now introduce the log-formulation proposed in [14]. We want to map solutions of the system (2.2) with solutions of another system of equations where a partial differential equation for the logarithm of the conformation tensor is substituted to the Oldroyd-B partial differential equation for the conformation tensor $\boldsymbol{\sigma}$.

In order to obtain a constitutive equation in terms of $\psi=\ln \sigma$, following [14], we make use of the following decomposition of the deformation tensor $\boldsymbol{\nabla} \boldsymbol{u} \in \mathbb{R}^{d \times d}$ (see Appendix C for a proof):

Lemma 2.3. For any matrix $\boldsymbol{\nabla} \boldsymbol{u}$ and any symmetric positive definite matrix $\boldsymbol{\sigma}$ in $\mathbb{R}^{d \times d}$, there exist in $\mathbb{R}^{d \times d}$ two antisymmetric matrices $\boldsymbol{\Omega}, \boldsymbol{N}$ and a symmetric matrix $\boldsymbol{B}$ that commutes with $\boldsymbol{\sigma}$, such that:

$$
\nabla \boldsymbol{u}=\boldsymbol{\Omega}+\boldsymbol{B}+\boldsymbol{N} \boldsymbol{\sigma}^{-1} .
$$

Moreover, we have $\operatorname{tr} \boldsymbol{\nabla} \boldsymbol{u}=\operatorname{tr} \boldsymbol{B}$.

We now proceed to the change of variable $\boldsymbol{\psi}=\ln \boldsymbol{\sigma}$. The system (2.2) then rewrites (see [14] for a proof):

$$
\left\{\begin{aligned}
& \operatorname{Re}\left(\frac{\partial \boldsymbol{u}}{\partial t}+\boldsymbol{u} \cdot \boldsymbol{\nabla} \boldsymbol{u}\right)=-\boldsymbol{\nabla} p+(1-\varepsilon) \Delta \boldsymbol{u}+\frac{\varepsilon}{\mathrm{Wi}} \operatorname{div} \mathrm{e}^{\psi}, \\
& \operatorname{div} \boldsymbol{u}=0, \\
& \frac{\partial \boldsymbol{\psi}}{\partial t}+(\boldsymbol{u} \cdot \boldsymbol{\nabla}) \boldsymbol{\psi}=\boldsymbol{\Omega} \boldsymbol{\psi}-\boldsymbol{\psi} \boldsymbol{\Omega}+2 \boldsymbol{B}+\frac{1}{\mathrm{Wi}}\left(\mathrm{e}^{-\boldsymbol{\psi}}-\boldsymbol{I}\right) .
\end{aligned}\right.
$$

It is supplied with unchanged initial and boundary conditions for $\boldsymbol{u}$, plus the initial condition $\boldsymbol{\psi}(t=0)=$ $\ln \boldsymbol{\sigma}(t=0)$ for the log-conformation tensor. 


\subsubsection{Reformulation of the free energy estimate}

A result similar to Theorem 2.2 can be obtained for system (2.10), where the free energy is written in terms of $\psi$ as:

The following theorem then holds:

$$
F\left(\boldsymbol{u}, \mathrm{e}^{\psi}\right)=\frac{\operatorname{Re}}{2} \int_{\mathcal{D}}|\boldsymbol{u}|^{2}+\frac{\varepsilon}{2 \mathrm{Wi}} \int_{\mathcal{D}} \operatorname{tr}\left(\mathrm{e}^{\psi}-\boldsymbol{\psi}-\boldsymbol{I}\right) .
$$

Theorem 2.4. Let $(\boldsymbol{u}, p, \boldsymbol{\psi})$ be a smooth solution to system (2.10) supplied with homogeneous Dirichlet boundary conditions for $\boldsymbol{u}$. The free energy satisfies:

$$
\frac{\mathrm{d}}{\mathrm{d} t} F\left(\boldsymbol{u}, \mathrm{e}^{\psi}\right)+(1-\varepsilon) \int_{\mathcal{D}}|\boldsymbol{\nabla} \boldsymbol{u}|^{2}+\frac{\varepsilon}{2 \mathrm{Wi}^{2}} \int_{\mathcal{D}} \operatorname{tr}\left(\mathrm{e}^{\psi}+\mathrm{e}^{-\boldsymbol{\psi}}-2 \boldsymbol{I}\right)=0 .
$$

From this estimate, we get that $F\left(\boldsymbol{u}, \mathrm{e}^{\psi}\right)$ decreases exponentially fast in time to zero.

Proof of Theorem (2.4). The proof of this theorem mimics the proof of Theorem 2.2. We go over the steps of the proof, and point out the differences with the previous case. Let $(\boldsymbol{u}, p, \boldsymbol{\psi})$ be a smooth solution to (2.10).

From the inner product of the momentum conservation equation in (2.10) with the velocity $\boldsymbol{u}$, we obtain:

$$
\frac{\operatorname{Re}}{2} \frac{\mathrm{d}}{\mathrm{d} t} \int_{\mathcal{D}}|\boldsymbol{u}|^{2}=-(1-\varepsilon) \int_{\mathcal{D}}|\nabla \boldsymbol{u}|^{2}-\frac{\varepsilon}{\mathrm{Wi}} \int_{\mathcal{D}} \boldsymbol{\nabla} \boldsymbol{u}: \mathrm{e}^{\psi},
$$

which is equivalent to (2.5). Taking the trace of the evolution equation for the conformation tensor, we get:

$$
\frac{\mathrm{d}}{\mathrm{d} t} \int_{\mathcal{D}} \operatorname{tr} \boldsymbol{\psi}=\frac{1}{\mathrm{Wi}} \int_{\mathcal{D}} \operatorname{tr}\left(\mathrm{e}^{-\boldsymbol{\psi}}-\boldsymbol{I}\right),
$$

which is equivalent to (2.8). Contracting the evolution equation for $\boldsymbol{\psi}$ with $\mathrm{e}^{\psi}$ and using (1.13) with $\boldsymbol{\psi}=\ln \boldsymbol{\sigma}$, we rewrite the first term of this inner product:

$$
\left(\frac{\partial \boldsymbol{\psi}}{\partial t}+\boldsymbol{u} \cdot \boldsymbol{\nabla} \psi\right): \mathrm{e}^{\psi}=\left(\frac{\partial}{\partial t}+\boldsymbol{u} \cdot \boldsymbol{\nabla}\right) \operatorname{tr} \mathrm{e}^{\psi} .
$$

Recall that the decomposition (2.9) of $\boldsymbol{\nabla} \boldsymbol{u}$ allows to rewrite the second term:

$$
\boldsymbol{\nabla} \boldsymbol{u}: \mathrm{e}^{\boldsymbol{\psi}}=\boldsymbol{\Omega}: \mathrm{e}^{\boldsymbol{\psi}}+\boldsymbol{B}: \mathrm{e}^{\boldsymbol{\psi}}+\left(\boldsymbol{N} \mathrm{e}^{-\boldsymbol{\psi}}\right): \mathrm{e}^{\boldsymbol{\psi}}=\boldsymbol{B}: \mathrm{e}^{\boldsymbol{\psi}},
$$

where we have used the symmetry of $\mathrm{e}^{\boldsymbol{\psi}}$ and the antisymmetry of $\boldsymbol{\Omega}$ and $\boldsymbol{N}$. Then, notice that, since $\boldsymbol{\psi}$ and $\mathrm{e}^{\psi}$ commute, we have:

$$
(\boldsymbol{\Omega} \psi-\boldsymbol{\psi} \boldsymbol{\Omega}): \mathrm{e}^{\psi}=\operatorname{tr}\left(\boldsymbol{\Omega} \psi \mathrm{e}^{\psi}\right)-\operatorname{tr}\left(\boldsymbol{\psi} \boldsymbol{\Omega} \mathrm{e}^{\psi}\right)=\operatorname{tr}\left(\boldsymbol{\Omega} \psi \mathrm{e}^{\psi}\right)-\operatorname{tr}\left(\boldsymbol{\Omega} \psi \mathrm{e}^{\psi}\right)=0,
$$

we finally obtain an equation equivalent to (2.6):

$$
\frac{\mathrm{d}}{\mathrm{d} t} \int_{\mathcal{D}} \operatorname{tr} \mathrm{e}^{\psi}=2 \int_{\mathcal{D}} \boldsymbol{\nabla} \boldsymbol{u}: \mathrm{e}^{\psi}-\frac{1}{\mathrm{Wi}} \int_{\mathcal{D}} \operatorname{tr}\left(\mathrm{e}^{\psi}-\boldsymbol{I}\right) .
$$

It is noticeable that in this proof, we made no use of the positivity of $\sigma=\mathrm{e}^{\boldsymbol{\psi}}$, in contrast to the proof of Theorem 2.2.

The combination $(2.13)-\frac{\varepsilon}{2 \mathrm{Wi}} \times(2.14)+\frac{\varepsilon}{2 \mathrm{Wi}} \times(2.17)$ gives $(2.12)$ :

$$
\frac{\mathrm{d}}{\mathrm{d} t}\left[\frac{\mathrm{Re}}{2} \int_{\mathcal{D}}|\boldsymbol{u}|^{2}+\frac{\varepsilon}{2 \mathrm{Wi}} \int_{\mathcal{D}} \operatorname{tr}\left(\mathrm{e}^{\psi}-\boldsymbol{\psi}-\boldsymbol{I}\right)\right]+(1-\varepsilon) \int_{\mathcal{D}}|\boldsymbol{\nabla} \boldsymbol{u}|^{2}+\frac{\varepsilon}{2 \mathrm{Wi}^{2}} \int_{\mathcal{D}} \operatorname{tr}\left(\mathrm{e}^{\psi}+\mathrm{e}^{-\boldsymbol{\psi}}-2 \boldsymbol{I}\right)=0 .
$$


This is exactly equivalent to (2.4). As in the proof of Theorem 2.2, we then obtain that $F\left(\boldsymbol{u}, \mathrm{e}^{\psi}\right)$ decreases exponentially fast in time to zero.

\section{Construction of numerical schemes with Scott-Vogelius finite elements FOR THE VELOCITY-PRESSURE FIELD $\left(\boldsymbol{u}_{h}, p_{h}\right)$}

We would now like to build numerical schemes for both systems of equations (2.2) and (2.10) that respectively preserve the dissipation properties of Theorems 2.2 and 2.4 for discrete free energies similar to (2.3) and (2.11). We first present discretizations that allow for a simple and complete exposition of our reasoning in order to derive discrete free energy estimates. Possible extensions will be discussed in Section 4.3 (other discretizations for the velocity-pressure field) and in Appendix D (higher-order discretizations for the stress field).

\subsection{Variational formulations of the problems}

To discretize (2.2) and (2.10) in space using a finite element method, we first write variational formulations for (2.2) and (2.10) that are satisfied by smooth solutions of the previous systems. Smooth solutions $(\boldsymbol{u}, p, \boldsymbol{\sigma})$ and $(\boldsymbol{u}, p, \boldsymbol{\psi})$ to system $(2.2)$ and (2.10) respectively satisfy the variational formulations:

$$
\begin{aligned}
0=\int_{\mathcal{D}}(\operatorname{Re} & \left(\frac{\partial \boldsymbol{u}}{\partial t}+\boldsymbol{u} \cdot \boldsymbol{\nabla} \boldsymbol{u}\right) \cdot \boldsymbol{v}+(1-\varepsilon) \boldsymbol{\nabla} \boldsymbol{u}: \boldsymbol{\nabla} \boldsymbol{v}+\frac{\varepsilon}{\mathrm{Wi}} \boldsymbol{\sigma}: \boldsymbol{\nabla} \boldsymbol{v}-p \operatorname{div} \boldsymbol{v}+q \operatorname{div} \boldsymbol{u} \\
& \left.+\left(\frac{\partial \boldsymbol{\sigma}}{\partial t}+\boldsymbol{u} \cdot \boldsymbol{\nabla} \boldsymbol{\sigma}\right): \boldsymbol{\phi}-\left((\boldsymbol{\nabla} \boldsymbol{u}) \boldsymbol{\sigma}+\boldsymbol{\sigma}(\boldsymbol{\nabla} \boldsymbol{u})^{T}\right): \boldsymbol{\phi}+\frac{1}{\mathrm{Wi}}(\boldsymbol{\sigma}-\boldsymbol{I}): \boldsymbol{\phi}\right)
\end{aligned}
$$

and

$$
\begin{aligned}
& 0=\int_{\mathcal{D}}( \operatorname{Re}\left(\frac{\partial \boldsymbol{u}}{\partial t}+\boldsymbol{u} \cdot \boldsymbol{\nabla} \boldsymbol{u}\right) \cdot \boldsymbol{v}+(1-\varepsilon) \boldsymbol{\nabla} \boldsymbol{u}: \boldsymbol{\nabla} \boldsymbol{v}+\frac{\varepsilon}{\mathrm{Wi}} \mathrm{e}^{\psi}: \boldsymbol{\nabla} \boldsymbol{v}-p \operatorname{div} \boldsymbol{v}+q \operatorname{div} \boldsymbol{u} \\
&\left.+\left(\frac{\partial \boldsymbol{\psi}}{\partial t}+\boldsymbol{u} \cdot \boldsymbol{\nabla} \psi\right): \boldsymbol{\phi}-(\boldsymbol{\Omega} \boldsymbol{\psi}-\boldsymbol{\psi} \boldsymbol{\Omega}): \boldsymbol{\phi}-2 \boldsymbol{B}: \boldsymbol{\phi}-\frac{1}{\mathrm{Wi}}\left(\mathrm{e}^{-\psi}-\boldsymbol{I}\right): \boldsymbol{\phi}\right)
\end{aligned}
$$

for all sufficiently regular test functions $(\boldsymbol{v}, q, \phi)$.

In this variational framework, we recover the free energy estimates (2.4) (respectively (2.12)) using the test functions $\left(\boldsymbol{u}, p, \frac{\varepsilon}{2 W \mathrm{i}}\left(\boldsymbol{I}-\boldsymbol{\sigma}^{-1}\right)\right)$ (respectively $\left.\left(\boldsymbol{u}, p, \frac{\varepsilon}{2 \mathrm{Wi}^{2}}\left(\mathrm{e}^{\boldsymbol{\psi}}-\boldsymbol{I}\right)\right)\right)$ in (3.1) (respectively (3.2)).

\subsection{Numerical schemes with Scott-Vogelius finite elements for $\left(u_{h}, p_{h}\right)$}

Using the Galerkin discretization method, we now want to build variational numerical integration schemes that are based on the variational formulations (3.1) and (3.2) using finite-dimensional approximations of the solution/test spaces. We will then show in the next Section 4 that solutions to these schemes satisfy discrete free energy estimates which are equivalent to those in Theorems 2.2 and 2.4.

First, the time interval $[0, T)$ is split into $N_{T}$ intervals $\left[t^{n}, t^{n+1}\right)$ of constant size $\Delta t=\frac{T}{N_{T}}$, with $t^{n}=n \Delta t$ for $n=0, \ldots, N_{T}$. For all $n=0, \ldots, N_{T}-1$, we denote by $\left(\boldsymbol{u}_{h}^{n}, p_{h}^{n}, \boldsymbol{\sigma}_{h}^{n}\right)\left(\operatorname{resp} .\left(\boldsymbol{u}_{h}^{n}, p_{h}^{n}, \boldsymbol{\psi}_{h}^{n}\right)\right)$, the value at time $t_{n}$ of the discrete solutions $\left(\boldsymbol{u}_{h}, p_{h}, \boldsymbol{\sigma}_{h}\right)$ (resp. $\left.\left(\boldsymbol{u}_{h}, p_{h}, \boldsymbol{\psi}_{h}\right)\right)$ in finite element spaces.

In all the following sections, we will assume that the domain $\mathcal{D}$ is polyhedral. We define a conformal mesh $\mathcal{T}_{h}$ built from a tessellation of the domain $\mathcal{D}$,

$$
\mathcal{T}_{h}=\bigcup_{k=1}^{N_{K}} K_{k}
$$

made of $N_{K}$ simplicial elements $K_{k}$ and $N_{D}$ nodes at the internal vertices. We denote by $h_{K_{k}}$ the diameter of the element $K_{k}$ and assume that the mesh is uniformly regular, with maximal diameter $h \geq \max _{1 \leq k \leq N_{K}} h_{K_{k}}$. 
For each element $K_{k}$ of the mesh $\mathcal{T}_{h}$, we denote by $\boldsymbol{n}_{K_{k}}$ the outward unitary normal vector to element $K_{k}$, defined on its boundary $\partial K_{k}$. We also denote by $\left\{E_{j} \mid j=1, \ldots, N_{E}\right\}$ the internal edges of the mesh $\mathcal{T}_{h}$ when $d=2$, or the faces of volume elements when $d=3$ (also termed as "edges" for the sake of simplicity in the following).

For the velocity-pressure field $\left(\boldsymbol{u}_{h}, p_{h}\right)$, we choose the mixed finite element space $\left(\mathbb{P}_{2}\right)^{d} \times \mathbb{P}_{1 \text {,disc }}$ of ScottVogelius [43], where:

- by $\boldsymbol{u}_{h} \in\left(\mathbb{P}_{2}\right)^{d}$ we mean that $\boldsymbol{u}_{h}$ is a vector field with entries over $\mathcal{D}$ that are continuous polynomials of maximal degree 2 ;

- and by $p_{h} \in \mathbb{P}_{1 \text {,disc }}$ we mean that $p_{h}$ is a scalar field with entries over $\mathcal{T}_{h}$ that are piecewise continuous polynomials of maximal degree 1 (thus discontinuous over $\mathcal{D}$ ).

This choice is very convenient to establish the free-energy estimates at the discrete level. As mentioned earlier, other choices will be discussed in Section 4.3. For general meshes, this finite element does not satisfy the Babuška-Brezzi inf-sup condition. However, for meshes built using a particular process based on a first mesh of macro-elements, this mixed finite element space is known to satisfy the Babuška-Brezzi inf-sup condition (this is detailed in [1] for instance). The interest of this finite element is that the velocity field is divergence-free:

$$
\operatorname{div} \boldsymbol{u}_{h}(\boldsymbol{x})=0, \forall \boldsymbol{x} \in \mathcal{D}
$$

because $\operatorname{div} \boldsymbol{u}_{h} \in \mathbb{P}_{1 \text {,disc }}$ can be used as a test function for the pressure field in the weak formulation of the incompressibility constraint $\int_{\mathcal{D}}\left(\operatorname{div} \boldsymbol{u}_{h}\right) q_{h}=0$.

For the approximation of $\boldsymbol{\sigma}_{h}$ and $\boldsymbol{\psi}_{h}$, we use discontinuous finite elements to derive the free energy estimates. For simplicity, we first consider piecewise constant approximations of $\boldsymbol{\sigma}_{h}$ and $\boldsymbol{\psi}_{h}$ in Sections 3 and 4 . In Appendix D, we will come back to this assumption and discuss the use of higher-order finite element spaces for $\boldsymbol{\sigma}_{h}$ and $\boldsymbol{\psi}_{h}$. All along this work, we denote by $\boldsymbol{\sigma}_{h} \in\left(\mathbb{P}_{0}\right)^{\frac{d(d+1)}{2}}$ the fact that the symmetric-tensor field $\boldsymbol{\sigma}_{h}$ is discretized using a $\frac{d(d+1)}{2}$-dimensional so-called stress field, which stands for the entries in $\mathbb{P}_{0}$ of a symmetric $(d \times d)$-dimensional tensor field, thus enforcing the symmetry in the discretization.

The advection terms $\boldsymbol{u} \cdot \boldsymbol{\nabla} \boldsymbol{\sigma}$ and $\boldsymbol{u} \cdot \boldsymbol{\nabla} \boldsymbol{\psi}$ will be discretized either through a characteristic method in the spirit of $[3,40,48]$, or with the discontinuous Galerkin (DG) method in the spirit of [23]. Notice already that the characteristic method requires the velocity field to be more regular than the discontinuous Galerkin method in order to define the flow associated with the vector field $\boldsymbol{u}_{h}$.

For the discontinuous Galerkin method, we will need the following notation. Let $E_{j}$ be some internal edge in the mesh $\mathcal{T}_{h}$. To each edge $E_{j}$, we associate a unitary orthogonal vector $\boldsymbol{n} \equiv \boldsymbol{n}_{E_{j}}$, whose orientation will not matter in the following. Then, for a given velocity field $\boldsymbol{u}_{h}$ in $\mathcal{D}$ that is well defined on the edges, for any variable $\phi$ in $\mathcal{D}$ and any interior point $\boldsymbol{x}$ to the edge $E_{j}$, we respectively define the downstream and upstream values of $\phi$ by:

$$
\phi^{+}(\boldsymbol{x})=\lim _{\delta \rightarrow 0^{+}} \phi\left(\boldsymbol{x}+\delta \boldsymbol{u}_{h}(\boldsymbol{x})\right) \text { and } \boldsymbol{\phi}^{-}(\boldsymbol{x})=\lim _{\delta \rightarrow 0^{-}} \phi\left(\boldsymbol{x}+\delta \boldsymbol{u}_{h}(\boldsymbol{x})\right) .
$$

We denote by $\llbracket \phi \rrbracket(\boldsymbol{x})=\boldsymbol{\phi}^{+}(\boldsymbol{x})-\boldsymbol{\phi}^{-}(\boldsymbol{x})$ the jump of $\boldsymbol{\phi}$ over the edge $E_{j}$ and by $\{\boldsymbol{\phi}\}(\boldsymbol{x})=\frac{\phi^{+}(\boldsymbol{x})+\phi^{-}(\boldsymbol{x})}{2}$ the mean value over the edge. Then, one can easily check the following formula for any function $\phi$ :

$$
\sum_{E_{j}} \int_{E_{j}}\left|\boldsymbol{u}_{h} \cdot \boldsymbol{n}\right| \llbracket \phi \rrbracket=-\sum_{K_{k}} \int_{\partial K_{k}}\left(\boldsymbol{u}_{h} \cdot \boldsymbol{n}_{K_{k}}\right) \phi .
$$

Let us now present in the next section the discrete variational formulations we will consider.

Remark 3.1. In what follows, we do not consider the possible instabilities occurring when advection dominates diffusion in the Navier-Stokes equation for the velocity field $\boldsymbol{u}_{h}$. Indeed, in practice, one typically considers small Reynolds number flows for polymeric fluids, so that we are in a regime where such instabilities are not observed. Moreover, we also assume that $0 \leq \varepsilon<1$ so that there is no problem of compatibilities between the discretization space for the velocity and for the stress (see [7] for more details). 


\subsection{Numerical schemes with $\sigma_{h}$ piecewise constant}

Variational formulations of the discrete problem write, for all $n=0, \ldots, N_{T}-1$, as follows: With the characteristic method: For a given $\left(\boldsymbol{u}_{h}^{n}, p_{h}^{n}, \boldsymbol{\sigma}_{h}^{n}\right)$, find $\left(\boldsymbol{u}_{h}^{n+1}, p_{h}^{n+1}, \boldsymbol{\sigma}_{h}^{n+1}\right) \in\left(\mathbb{P}_{2}\right)^{d} \times \mathbb{P}_{1, \text { disc }} \times\left(\mathbb{P}_{0}\right)^{\frac{d(d+1)}{2}}$ such that, for any test function $(\boldsymbol{v}, q, \phi) \in\left(\mathbb{P}_{2}\right)^{d} \times \mathbb{P}_{1, \text { disc }} \times\left(\mathbb{P}_{0}\right)^{\frac{d(d+1)}{2}}$,

$$
\begin{aligned}
0=\int_{\mathcal{D}} & \operatorname{Re}\left(\frac{\boldsymbol{u}_{h}^{n+1}-\boldsymbol{u}_{h}^{n}}{\Delta t}+\boldsymbol{u}_{h}^{n} \cdot \nabla \boldsymbol{u}_{h}^{n+1}\right) \cdot \boldsymbol{v}-p_{h}^{n+1} \operatorname{div} \boldsymbol{v}+q \operatorname{div} \boldsymbol{u}_{h}^{n+1}+(1-\varepsilon) \boldsymbol{\nabla} \boldsymbol{u}_{h}^{n+1}: \nabla \boldsymbol{v}+\frac{\varepsilon}{\mathrm{Wi}} \boldsymbol{\sigma}_{h}^{n+1}: \nabla \boldsymbol{v} \\
& +\left(\frac{\boldsymbol{\sigma}_{h}^{n+1}-\boldsymbol{\sigma}_{h}^{n} \circ X^{n}\left(t^{n}\right)}{\Delta t}\right): \boldsymbol{\phi}-\left(\left(\boldsymbol{\nabla} \boldsymbol{u}_{h}^{n+1}\right) \boldsymbol{\sigma}_{h}^{n+1}+\boldsymbol{\sigma}_{h}^{n+1}\left(\boldsymbol{\nabla} \boldsymbol{u}_{h}^{n+1}\right)^{T}\right): \boldsymbol{\phi}+\frac{1}{\mathrm{Wi}}\left(\boldsymbol{\sigma}_{h}^{n+1}-\boldsymbol{I}\right): \boldsymbol{\phi} . \quad(3.6)
\end{aligned}
$$

This problem is supplied with an initial condition $\left(\boldsymbol{u}_{h}^{0}, p_{h}^{0}, \boldsymbol{\sigma}_{h}^{0}\right) \in\left(\mathbb{P}_{2}\right)^{d} \times \mathbb{P}_{1, \text { disc }} \times\left(\mathbb{P}_{0}\right)^{\frac{d(d+1)}{2}}$.

The function $X^{n}(t): x \in \mathcal{D} \mapsto X^{n}(t, x) \in \mathcal{D}$ is the "backward" flow associated with the velocity field $\boldsymbol{u}_{h}^{n}$ and satisfies, for all $x \in \mathcal{D}$ :

$$
\left\{\begin{array}{l}
\frac{\mathrm{d}}{\mathrm{d} t} X^{n}(t, x)=\boldsymbol{u}_{h}^{n}\left(X^{n}(t, x)\right), \quad \forall t \in\left[t^{n}, t^{n+1}\right], \\
X^{n}\left(t^{n+1}, x\right)=x
\end{array}\right.
$$

With the discontinuous Galerkin method: For a given $\left(\boldsymbol{u}_{h}^{n}, p_{h}^{n}, \boldsymbol{\sigma}_{h}^{n}\right)$, find $\left(\boldsymbol{u}_{h}^{n+1}, p_{h}^{n+1}, \boldsymbol{\sigma}_{h}^{n+1}\right) \in\left(\mathbb{P}_{2}\right)^{d} \times \mathbb{P}_{1, \text { disc }} \times$ $\left(\mathbb{P}_{0}\right)^{\frac{d(d+1)}{2}}$ such that, for any test function $(\boldsymbol{v}, q, \phi) \in\left(\mathbb{P}_{2}\right)^{d} \times \mathbb{P}_{1, \text { disc }} \times\left(\mathbb{P}_{0}\right)^{\frac{d(d+1)}{2}}$,

$$
\begin{aligned}
& 0=\sum_{k=1}^{N_{K}} \int_{K_{k}} \operatorname{Re}\left(\frac{\boldsymbol{u}_{h}^{n+1}-\boldsymbol{u}_{h}^{n}}{\Delta t}+\boldsymbol{u}_{h}^{n} \cdot \nabla \boldsymbol{u}_{h}^{n+1}\right) \cdot \boldsymbol{v}-p_{h}^{n+1} \operatorname{div} \boldsymbol{v}+q \operatorname{div} \boldsymbol{u}_{h}^{n+1}+(1-\varepsilon) \boldsymbol{\nabla} \boldsymbol{u}_{h}^{n+1}: \boldsymbol{\nabla} \boldsymbol{v}+\frac{\varepsilon}{\mathrm{Wi}} \boldsymbol{\sigma}_{h}^{n+1}: \nabla \boldsymbol{v} \\
&+\left(\frac{\boldsymbol{\sigma}_{h}^{n+1}-\boldsymbol{\sigma}_{h}^{n}}{\Delta t}\right): \boldsymbol{\phi}-\left(\left(\boldsymbol{\nabla} \boldsymbol{u}_{h}^{n+1}\right) \boldsymbol{\sigma}_{h}^{n+1}+\boldsymbol{\sigma}_{h}^{n+1}\left(\boldsymbol{\nabla} \boldsymbol{u}_{h}^{n+1}\right)^{T}\right): \boldsymbol{\phi}+\frac{1}{\mathrm{Wi}}\left(\boldsymbol{\sigma}_{h}^{n+1}-\boldsymbol{I}\right): \boldsymbol{\phi} \\
&+\sum_{j=1}^{N_{E}} \int_{E_{j}}\left|\boldsymbol{u}_{h}^{n} \cdot \boldsymbol{n}\right| \llbracket \boldsymbol{\sigma}_{h}^{n+1} \rrbracket: \boldsymbol{\phi}^{+} \cdot(3.8)
\end{aligned}
$$

Since $\boldsymbol{\sigma}_{h} \in\left(\mathbb{P}_{0}\right)^{\frac{d(d+1)}{2}}$ is discontinuous, we have discretized the advection term for $\boldsymbol{\sigma}_{h}$ with a sum of jumps similar to the usual upwind technique, where $\phi^{+}=\left(\frac{1}{2} \llbracket \phi \rrbracket+\{\phi\}\right)$ (see $\left.[13,23]\right)$.

Remark 3.2. In all the following, we assume that, when using the characteristic method:

- the characteristics are exactly integrated;

- and the integrals involving the backward flow $X^{n}$ are exactly computed.

We are aware of the fact that these assumptions are strong, and that numerical instabilities may be induced by bad integration schemes $[38,44]$. Hence, considering the lack for an analysis of those integration schemes for the characteristics in the present study, our analysis of discontinuous Galerkin discretizations of the advection terms may seem closer to the real implementation than that of the discretizations using the characteristic method.

\subsection{Numerical schemes with $\psi_{h}$ piecewise constant}

We now show how to discretize the variational log-formulation similarly as above. For this, we will need the following elementwise decomposition of the velocity gradient (see Lem. 2.3 above):

$$
\nabla \boldsymbol{u}_{h}^{n+1}=\boldsymbol{\Omega}_{h}^{n+1}+\boldsymbol{B}_{h}^{n+1}+\boldsymbol{N}_{h}^{n+1} \mathrm{e}^{-\boldsymbol{\psi}_{h}^{n+1}}
$$

Moreover, for the decomposition (3.9) with $\boldsymbol{u} \in\left(\mathbb{P}_{2}\right)^{d}$, we will need the following Lemma 3.3 for $k=1$, which is proved in Appendix C: 
Lemma 3.3. Let $\nabla \boldsymbol{u}_{h}^{n+1} \in\left(\mathbb{P}_{k, \text { disc }}\right)^{d \times d}$ for some $k \in \mathbb{N}$. Then, for any symmetric positive definite matrix $\mathrm{e}^{\psi_{h}^{n+1}} \in\left(\mathbb{P}_{0}\right)^{\frac{d(d+1)}{2}}$, there exist two antisymmetric matrices $\boldsymbol{\Omega}_{h}^{n+1}, \boldsymbol{N}_{h}^{n+1} \in\left(\mathbb{P}_{k, \text { disc }}\right)^{\frac{d(d-1)}{2}}$ and a symmetric matrix $\boldsymbol{B}_{h}^{n+1} \in\left(\mathbb{P}_{k, \text { disc }}\right)^{\frac{d(d+1)}{2}}$ that commutes with $\mathrm{e}_{h}^{\psi_{h}^{n+1}}$, such that the matrix-valued function $\boldsymbol{\nabla} \boldsymbol{u}_{h}^{n+1}$ can be decomposed pointwise as: $\boldsymbol{\nabla} \boldsymbol{u}_{h}^{n+1}=\boldsymbol{\Omega}_{h}^{n+1}+\boldsymbol{B}_{h}^{n+1}+\boldsymbol{N}_{h}^{n+1} \mathrm{e}^{-\boldsymbol{\psi}_{h}^{n+1}}$.

Variational formulations of the discrete problem write, for all $n=0, \ldots, N_{T}-1$, as follows: With the characteristic method: For a given $\left(\boldsymbol{u}_{h}^{n}, p_{h}^{n}, \boldsymbol{\psi}_{h}^{n}\right)$, find $\left(\boldsymbol{u}_{h}^{n+1}, p_{h}^{n+1}, \boldsymbol{\psi}_{h}^{n+1}\right) \in\left(\mathbb{P}_{2}\right)^{d} \times \mathbb{P}_{1, \text { disc }} \times\left(\mathbb{P}_{0}\right)^{\frac{d(d+1)}{2}}$ such that, for any test function $(\boldsymbol{v}, q, \phi) \in\left(\mathbb{P}_{2}\right)^{d} \times \mathbb{P}_{1, \text { disc }} \times\left(\mathbb{P}_{0}\right)^{\frac{d(d+1)}{2}}$,

$$
\begin{aligned}
0 & =\int_{\mathcal{D}} \operatorname{Re}\left(\frac{\boldsymbol{u}_{h}^{n+1}-\boldsymbol{u}_{h}^{n}}{\Delta t}+\boldsymbol{u}_{h}^{n} \cdot \boldsymbol{\nabla} \boldsymbol{u}_{h}^{n+1}\right) \cdot \boldsymbol{v}-p_{h}^{n+1} \operatorname{div} \boldsymbol{v}+q \operatorname{div} \boldsymbol{u}_{h}^{n+1}+(1-\varepsilon) \boldsymbol{\nabla} \boldsymbol{u}_{h}^{n+1}: \nabla \boldsymbol{v}+\frac{\varepsilon}{\mathrm{Wi}} \mathrm{e}_{h}^{\psi^{n+1}}: \boldsymbol{\nabla} \boldsymbol{v} \\
& +\left(\frac{\boldsymbol{\psi}_{h}^{n+1}-\boldsymbol{\psi}_{h}^{n} \circ X^{n}\left(t^{n}\right)}{\Delta t}\right): \boldsymbol{\phi}-\left(\boldsymbol{\Omega}_{h}^{n+1} \boldsymbol{\psi}_{h}^{n+1}-\boldsymbol{\psi}_{h}^{n+1} \boldsymbol{\Omega}_{h}^{n+1}\right): \boldsymbol{\phi}-2 \boldsymbol{B}_{h}^{n+1}: \boldsymbol{\phi}-\frac{1}{\mathrm{Wi}}\left(\mathrm{e}^{-\boldsymbol{\psi}_{h}^{n+1}}-\boldsymbol{I}\right): \boldsymbol{\phi}, \quad(3.10)
\end{aligned}
$$

where the initial condition $\left(\boldsymbol{u}_{h}^{0}, p_{h}^{0}, \boldsymbol{\psi}_{h}^{0}\right) \in\left(\mathbb{P}_{2}\right)^{d} \times \mathbb{P}_{1, \text { disc }} \times\left(\mathbb{P}_{0}\right)^{\frac{d(d+1)}{2}}$ is given and where $X^{n}(t)$ is again defined by (3.7).

With the discontinuous Galerkin method: For a given $\left(\boldsymbol{u}_{h}^{n}, p_{h}^{n}, \boldsymbol{\psi}_{h}^{n}\right)$, find $\left(\boldsymbol{u}_{h}^{n+1}, p_{h}^{n+1}, \boldsymbol{\psi}_{h}^{n+1}\right) \in\left(\mathbb{P}_{2}\right)^{d} \times \mathbb{P}_{1, \text { disc }} \times$ $\left(\mathbb{P}_{0}\right)^{\frac{d(d+1)}{2}}$ such that, for any test function $(\boldsymbol{v}, q, \phi) \in\left(\mathbb{P}_{2}\right)^{d} \times \mathbb{P}_{1, \text { disc }} \times\left(\mathbb{P}_{0}\right)^{\frac{d(d+1)}{2}}$,

$$
\begin{array}{r}
0=\sum_{k=1}^{N_{K}} \int_{K_{k}} \operatorname{Re}\left(\frac{\boldsymbol{u}_{h}^{n+1}-\boldsymbol{u}_{h}^{n}}{\Delta t}+\boldsymbol{u}_{h}^{n} \cdot \boldsymbol{\nabla} \boldsymbol{u}_{h}^{n+1}\right) \cdot \boldsymbol{v}-p_{h}^{n+1} \operatorname{div} \boldsymbol{v}+q \operatorname{div} \boldsymbol{u}_{h}^{n+1}+(1-\varepsilon) \boldsymbol{\nabla} \boldsymbol{u}_{h}^{n+1}: \boldsymbol{\nabla} \boldsymbol{v}+\frac{\varepsilon}{\mathrm{Wi}} \mathrm{e}_{h}^{n+1}: \boldsymbol{\nabla} \boldsymbol{v} \\
+\left(\frac{\boldsymbol{\psi}_{h}^{n+1}-\boldsymbol{\psi}_{h}^{n}}{\Delta t}\right): \boldsymbol{\phi}-\left(\boldsymbol{\Omega}_{h}^{n+1} \boldsymbol{\psi}_{h}^{n+1}-\boldsymbol{\psi}_{h}^{n+1} \boldsymbol{\Omega}_{h}^{n+1}\right): \boldsymbol{\phi}-2 \boldsymbol{B}_{h}^{n+1}: \boldsymbol{\phi}-\frac{1}{\mathrm{Wi}}\left(\mathrm{e}^{-\boldsymbol{\psi}_{h}^{n+1}}-\boldsymbol{I}\right): \boldsymbol{\phi} \\
+\sum_{j=1}^{N_{E}} \int_{E_{j}}\left|\boldsymbol{u}_{h}^{n} \cdot \boldsymbol{n}\right| \llbracket \boldsymbol{\psi}_{h}^{n+1} \rrbracket: \boldsymbol{\phi}^{+} .
\end{array}
$$

Remark 3.4. Notice that the numerical schemes we propose are nonlinear due to the implicit terms corresponding to the discretization of the upper-convective derivative $(\boldsymbol{\nabla} \boldsymbol{u}) \boldsymbol{\sigma}+\boldsymbol{\sigma}(\boldsymbol{\nabla} \boldsymbol{u})^{T}$ (resp. $\left.\boldsymbol{\Omega} \boldsymbol{\psi}-\boldsymbol{\psi} \boldsymbol{\Omega}\right)$. In practice, this nonlinear system can be solved by fixed point procedures, either using the values at the previous time step as an initial guess, or using a predictor obtained by solving another scheme where the nonlinear terms are explicited.

\subsection{Local existence and uniqueness of the discrete solutions}

Before we show how to recover free energy estimates at the discrete level, let us now deal with the local-in-time existence and uniqueness of solutions to the discrete problems presented above.

First, since the mixed finite element space of Scott-Vogelius chosen in the systems above for the velocitypressure field satisfies the Babuška-Brezzi inf-sup condition, notice that the system (3.6) is equivalent to the following for all $n=0, \ldots, N_{T}-1$ : For a given $\left(\boldsymbol{u}_{h}^{n}, \boldsymbol{\sigma}_{h}^{n}\right)$, find $\left(\boldsymbol{u}_{h}^{n+1}, \boldsymbol{\sigma}_{h}^{n+1}\right) \in\left(\mathbb{P}_{2}\right)_{\mathrm{div}=0}^{d} \times\left(\mathbb{P}_{0}\right)^{\frac{d(d+1)}{2}}$ such that, for any test function $(\boldsymbol{v}, \phi) \in\left(\mathbb{P}_{2}\right)_{\operatorname{div}=0}^{d} \times\left(\mathbb{P}_{0}\right)^{\frac{d(d+1)}{2}}$,

$$
\begin{aligned}
0= & \int_{\mathcal{D}} \operatorname{Re}\left(\frac{\boldsymbol{u}_{h}^{n+1}-\boldsymbol{u}_{h}^{n}}{\Delta t}+\boldsymbol{u}_{h}^{n} \cdot \boldsymbol{\nabla} \boldsymbol{u}_{h}^{n+1}\right) \cdot \boldsymbol{v}+(1-\varepsilon) \boldsymbol{\nabla} \boldsymbol{u}_{h}^{n+1}: \boldsymbol{\nabla} \boldsymbol{v}+\frac{\varepsilon}{\mathrm{Wi}} \boldsymbol{\sigma}_{h}^{n+1}: \boldsymbol{\nabla} \boldsymbol{v} \\
& +\left(\frac{\boldsymbol{\sigma}_{h}^{n+1}-\boldsymbol{\sigma}_{h}^{n} \circ X^{n}\left(t^{n}\right)}{\Delta t}\right): \boldsymbol{\phi}-\left(\left(\boldsymbol{\nabla} \boldsymbol{u}_{h}^{n+1}\right) \boldsymbol{\sigma}_{h}^{n+1}+\boldsymbol{\sigma}_{h}^{n+1}\left(\boldsymbol{\nabla} \boldsymbol{u}_{h}^{n+1}\right)^{T}\right): \boldsymbol{\phi}+\frac{1}{\mathrm{Wi}}\left(\boldsymbol{\sigma}_{h}^{n+1}-\boldsymbol{I}\right): \boldsymbol{\phi},
\end{aligned}
$$


where the flow $X^{n}(t)$ is defined by (3.7) and where we have used the following notation:

$$
\left(\mathbb{P}_{2}\right)_{\text {div }=0}^{d}=\left\{\boldsymbol{v} \in\left(\mathbb{P}_{2}\right)^{d}, \int_{\mathcal{D}} q \operatorname{div} \boldsymbol{v}=0, \forall q \in \mathbb{P}_{1, \text { disc }}\right\}
$$

Notice that it is also straightforward to rewrite the systems (3.8), (3.10) and (3.11) using $\boldsymbol{u}_{h} \in\left(\mathbb{P}_{2}\right)_{\text {div=0 }}^{d}$

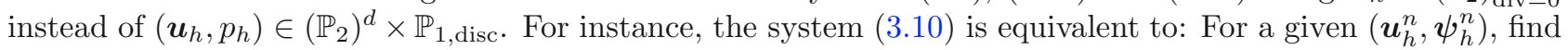
$\left(\boldsymbol{u}_{h}^{n+1}, \boldsymbol{\psi}_{h}^{n+1}\right) \in\left(\mathbb{P}_{2}\right)_{\text {div }=0}^{d} \times\left(\mathbb{P}_{0}\right)^{\frac{d(d+1)}{2}}$ such that, for all $(\boldsymbol{v}, \phi) \in\left(\mathbb{P}_{2}\right)_{\text {div }=0}^{d} \times\left(\mathbb{P}_{0}\right)^{\frac{d(d+1)}{2}}$,

$$
\begin{aligned}
0 & =\int_{\mathcal{D}} \operatorname{Re}\left(\frac{\boldsymbol{u}_{h}^{n+1}-\boldsymbol{u}_{h}^{n}}{\Delta t}+\boldsymbol{u}_{h}^{n} \cdot \boldsymbol{\nabla} \boldsymbol{u}_{h}^{n+1}\right) \cdot \boldsymbol{v}+(1-\varepsilon) \boldsymbol{\nabla} \boldsymbol{u}_{h}^{n+1}: \boldsymbol{\nabla} \boldsymbol{v}+\frac{\varepsilon}{\mathrm{Wi}} \mathrm{e}^{\boldsymbol{\psi}_{h}^{n+1}}: \boldsymbol{\nabla} \boldsymbol{v} \\
& +\left(\frac{\boldsymbol{\psi}_{h}^{n+1}-\boldsymbol{\psi}_{h}^{n} \circ X^{n}\left(t^{n}\right)}{\Delta t}\right): \boldsymbol{\phi}-\left(\boldsymbol{\Omega}_{h}^{n+1} \boldsymbol{\psi}_{h}^{n+1}-\boldsymbol{\psi}_{h}^{n+1} \boldsymbol{\Omega}_{h}^{n+1}\right): \boldsymbol{\phi}-2 \boldsymbol{B}_{h}^{n+1}: \boldsymbol{\phi}-\frac{1}{\mathrm{Wi}}\left(\mathrm{e}^{-\boldsymbol{\psi}_{h}^{n+1}}-\boldsymbol{I}\right): \boldsymbol{\phi}
\end{aligned}
$$

Then, we have the:

Proposition 3.5. Assume Scott-Vogelius finite elements are used for velocity-pressure, and piecewise constant discretization for the stress. For any couple $\left(\boldsymbol{u}_{h}^{n}, \boldsymbol{\sigma}_{h}^{n}\right)$ with $\boldsymbol{\sigma}_{h}^{n}$ symmetric positive definite, there exists $c_{0} \equiv$ $c_{0}\left(\boldsymbol{u}_{h}^{n}, \boldsymbol{\sigma}_{h}^{n}\right)>0$ such that, for all $0 \leq \Delta t<c_{0}$, there exists a unique solution $\left(\boldsymbol{u}_{h}^{n+1}, \boldsymbol{\sigma}_{h}^{n+1}\right)$ to the system (3.6) (resp. (3.8)) with $\boldsymbol{\sigma}_{h}^{n+1}$ symmetric positive definite.

Proof of Proposition 3.5. The proofs for systems (3.6) and (3.8) are similar, so we will proceed with the proof for system (3.6) only, using its restatement as system (3.12).

For a given mesh $\mathcal{T}_{h}$, let us denote by $Y^{n+1} \in \mathbb{R}^{2 N_{D}+3 N_{K}}$ the vector whose entries are respectively the nodal and elementwise values of $\left(\boldsymbol{u}_{h}^{n+1}, \boldsymbol{\sigma}_{h}^{n+1}\right)$, solution to the system (3.12). The system of equations (3.12) rewrites in terms of the vector $Y^{n+1} \in \mathbb{R}^{2 N_{D}+3 N_{K}}$ as: for a given $Y^{n}$ and $\Delta t$, find a zero $Y^{n+1}$ of the function $Q$ defined by

$$
Q\left(\Delta t, Y^{n+1}\right)=\Delta t A\left(Y^{n+1}\right) Y^{n+1}+\Delta t B\left(Y^{n}\right) Y^{n+1}+Y^{n+1}-C\left(Y^{n}, \Delta t\right)
$$

where $A$ and $B$ are linear continuous matrix-valued functions in $\mathbb{R}^{\left(2 N_{D}+3 N_{K}\right) \times\left(2 N_{D}+3 N_{K}\right)}$, and where $C$ is a vector-valued function in $\mathbb{R}^{2 N_{D}+3 N_{K}}$ (notice that the dependence of the function $C$ on $\Delta t$ is only related to the computation of the backward flow during a time step $\Delta t$, so that $C\left(Y^{n}, 0\right)=Y^{n}$, and with the DG method it simplifies as $\left.C\left(Y^{n}, \Delta t\right)=Y^{n}\right)$. The functions $A, B$ and $C$ also implicitly depend on $\mathcal{T}_{h}$, as well as on the parameters $\mathrm{Re}, \mathrm{Wi}, \varepsilon$.

Now, $Q(\Delta t, Y)$ is continuously differentiable with respect to $(\Delta t, Y)$ and we have, with $I$ the identity matrix in $\mathbb{R}^{\left(2 N_{D}+3 N_{K}\right) \times\left(2 N_{D}+3 N_{K}\right)}$ :

$$
\nabla_{Y} Q(\Delta t, Y)=I+\Delta t\left(B\left(Y^{n}\right)+A(Y)+\left(\nabla_{Y} A\right) Y\right) .
$$

Then, for given vectors $Y^{n}$ and $Y$, the matrix $\nabla_{Y} Q(\Delta t, Y)$ is invertible for all $\Delta t$ such that:

$$
0 \leq \Delta t \leq\left\|B\left(Y^{n}\right)+A(Y)+\left(\nabla_{Y} A\right) Y\right\|^{-1}
$$

(with convention $\left\|B\left(Y^{n}\right)+A(Y)+\left(\nabla_{Y} A\right) Y\right\|^{-1}=\infty$ if $B\left(Y^{n}\right)+A(Y)+\left(\nabla_{Y} A\right) Y=0$ ), and then defines an isomorphism in $\mathbb{R}^{2 N_{D}+3 N_{K}}$.

Let us denote by $S_{+}^{*}$ the subset of $\mathbb{R}^{2 N_{D}+3 N_{K}}$ that only contains vectors corresponding to elementwise values of positive definite matrix-valued functions $\sigma_{h}$ in $\mathcal{D}$. Since $\mathcal{S}_{+}^{*}\left(\mathbb{R}^{d \times d}\right)$ is an open (convex) domain of $\mathbb{R}^{d \times d}, S_{+}^{*}$ is clearly an open (convex) domain of $\mathbb{R}^{2 N_{D}+3 N_{K}}$.

Since $Q\left(0, Y^{n}\right)=0$ and $\nabla_{Y} Q\left(0, Y^{n}\right)$ is invertible, by virtue of the implicit function theorem, there exist a neighborhood $\left[0, c_{0}\right) \times V\left(Y^{n}\right)$ of $\left(0, Y^{n}\right)$ in $\mathbb{R}_{+} \cap S_{+}^{*}$ and a continuously differentiable function $R:\left[0, c_{0}\right) \rightarrow V\left(Y^{n}\right)$, 
such that, for all $0 \leq \Delta t<c_{0}$ :

$$
Y=R(\Delta t) \Longleftrightarrow Q(\Delta t, Y)=0 .
$$

For a given time step $\Delta t \in\left[0, c_{0}\right)$ and a given symmetric positive definite tensor field $\boldsymbol{\sigma}_{h}^{n}, R(\Delta t) \in V\left(Y^{n}\right)$ is the vector of values $Y^{n+1}$ for a solution $\left(\boldsymbol{u}_{h}^{n+1}, \boldsymbol{\sigma}_{h}^{n+1}\right)$ to the system (3.12) with a symmetric positive definite matrix $\boldsymbol{\sigma}_{h}^{n+1}$. Notice that, up to this point, $c_{0}=c_{0}\left(Y^{n}\right)$ is function of $Y^{n}$, as well as $\operatorname{Re}, \mathrm{Wi}, \varepsilon$ and $\mathcal{T}_{h}$.

For solutions $\left(\boldsymbol{u}_{h}^{n}, \boldsymbol{\sigma}_{h}^{n}\right)$ to the systems (3.10) and (3.11), we similarly have:

Proposition 3.6. Assume Scott-Vogelius finite elements are used for velocity-pressure, and piecewise constant discretization for the stress. Then, for any couple $\left(\boldsymbol{u}_{h}^{n}, \boldsymbol{\psi}_{h}^{n}\right)$, there exists a constant $c_{0} \equiv c_{0}\left(\boldsymbol{u}_{h}^{n}, \boldsymbol{\psi}_{h}^{n}\right)>0$ such that, for all $0 \leq \Delta t<c_{0}$, there exists a unique solution $\left(\boldsymbol{u}_{h}^{n+1}, \boldsymbol{\psi}_{h}^{n+1}\right)$ to the system (3.10) (resp. (3.11)).

The proof of Proposition 3.6 is similar to that of the Proposition 3.5, but for the expressions of $Q(\Delta t, Y)$ with respect to $Y$. An additional term $\Delta t D(Y)$ appears in $Q$ due to $\mathrm{e}^{\psi_{h}^{n+1}}$. This term is continuously differentiable with respect to $Y$, and the derivative $\nabla_{Y} Q\left(0, Y^{n}\right)$ is still invertible. Thus, the proof can be completed using similar arguments.

Anticipating the results of Section 5, we would like to mention that the above results will be extended in two directions, using the discrete free energy estimates which will be proved in the following.

- We will show that the constant $c_{0}$ in Proposition 3.5 (resp. Prop. 3.6) can be chosen independently of $\left(\boldsymbol{u}_{h}^{n}, \boldsymbol{\sigma}_{h}^{n}\right)\left(\right.$ resp. $\left.\left(\boldsymbol{u}_{h}^{n}, \boldsymbol{\psi}_{h}^{n}\right)\right)$, which yields a long-time existence and uniqueness result for the solutions to the discrete problems (see Props. 5.2 and 5.3 below). Of course, the limiting timestep will still depend on the parameters $\mathrm{Re}, \mathrm{Wi}, \varepsilon$ and on the mesh $\mathcal{T}_{h}$.

- We will also show, but for the log-formulation only, that it is possible to prove a long-time existence result without any restriction on the time step $\Delta t$ (see Prop. 5.4 below).

\section{Discrete free energy estimates With Piecewise CONStant discretization OF THE STRESS FIELDS $\boldsymbol{\sigma}_{h}$ AND $\boldsymbol{\psi}_{h}$}

In this section, we prove that various numerical schemes with piecewise constant $\boldsymbol{\sigma}_{h}$ or $\boldsymbol{\psi}_{h}$ satisfy a discrete free energy estimate. We first concentrate on Scott-Vogelius finite element spaces for $\left(\boldsymbol{u}_{h}, p_{h}\right)$ (introduced in Sect. 3) and then address the case of other mixed finite element spaces in Section 4.3.

\subsection{Free energy estimates with piecewise constant discretization of $\sigma_{h}$}

\subsubsection{The characteristic method}

Proposition 4.1. Let $\left(\boldsymbol{u}_{h}^{n}, p_{h}^{n}, \boldsymbol{\sigma}_{h}^{n}\right)_{0 \leq n \leq N_{T}}$ be a solution to (3.6), such that $\boldsymbol{\sigma}_{h}^{n}$ is positive definite. Then, the free energy of the solution $\left(\boldsymbol{u}_{h}^{n}, p_{h}^{n}, \boldsymbol{\sigma}_{h}^{n}\right)$ :

$$
F_{h}^{n}=F\left(\boldsymbol{u}_{h}^{n}, \boldsymbol{\sigma}_{h}^{n}\right)=\frac{\operatorname{Re}}{2} \int_{\mathcal{D}}\left|\boldsymbol{u}_{h}^{n}\right|^{2}+\frac{\varepsilon}{2 \mathrm{Wi}} \int_{\mathcal{D}} \operatorname{tr}\left(\boldsymbol{\sigma}_{h}^{n}-\ln \boldsymbol{\sigma}_{h}^{n}-\boldsymbol{I}\right),
$$

satisfies:

$$
F_{h}^{n+1}-F_{h}^{n}+\int_{\mathcal{D}} \frac{\operatorname{Re}}{2}\left|\boldsymbol{u}_{h}^{n+1}-\boldsymbol{u}_{h}^{n}\right|^{2}+\Delta t \int_{\mathcal{D}}(1-\varepsilon)\left|\nabla \boldsymbol{u}_{h}^{n+1}\right|^{2}+\frac{\varepsilon}{2 \mathrm{Wi}^{2}} \operatorname{tr}\left(\boldsymbol{\sigma}_{h}^{n+1}+\left(\boldsymbol{\sigma}_{h}^{n+1}\right)^{-1}-2 I\right) \leq 0 .
$$

In particular, the sequence $\left(F_{h}^{n}\right)_{0 \leq n \leq N_{T}}$ is non-increasing. 
Proof of Proposition 4.1. Let $\left(\boldsymbol{u}_{h}^{n+1}, p_{h}^{n+1}, \boldsymbol{\sigma}_{h}^{n+1}\right)$ be a solution to system (3.6). Notice that $\left(\boldsymbol{\sigma}_{h}^{n+1}\right)^{-1}$ is still in $\left(\mathbb{P}_{0}\right)^{\frac{d(d+1)}{2}}$. We can thus use $\left(\boldsymbol{u}_{h}^{n+1}, p_{h}^{n+1}, \frac{\varepsilon}{2 \mathrm{Wi}}\left(\boldsymbol{I}-\left(\boldsymbol{\sigma}_{h}^{n+1}\right)^{-1}\right)\right)$ as a test function in the system (3.6), which yields:

$$
\begin{aligned}
0=\int_{\mathcal{D}} \operatorname{Re}\left(\frac{\left|\boldsymbol{u}_{h}^{n+1}\right|^{2}-\left|\boldsymbol{u}_{h}^{n}\right|^{2}}{2 \Delta t}+\frac{\left|\boldsymbol{u}_{h}^{n+1}-\boldsymbol{u}_{h}^{n}\right|^{2}}{2 \Delta t}+\boldsymbol{u}_{h}^{n} \cdot \nabla \frac{\left|\boldsymbol{u}_{h}^{n+1}\right|^{2}}{2}\right) \\
+(1-\varepsilon)\left|\nabla \boldsymbol{u}_{h}^{n+1}\right|^{2}+\frac{\varepsilon}{\mathrm{Wi}} \boldsymbol{\sigma}_{h}^{n+1}: \boldsymbol{\nabla} \boldsymbol{u}_{h}^{n+1}+\frac{\varepsilon}{2 \mathrm{Wi}}\left[\left(\frac{\boldsymbol{\sigma}_{h}^{n+1}-\boldsymbol{\sigma}_{h}^{n} \circ X^{n}\left(t^{n}\right)}{\Delta t}\right):\left(\boldsymbol{I}-\left(\boldsymbol{\sigma}_{h}^{n+1}\right)^{-1}\right)\right. \\
\left.\quad-2\left(\boldsymbol{\nabla} \boldsymbol{u}_{h}^{n+1}\right) \boldsymbol{\sigma}_{h}^{n+1}:\left(\boldsymbol{I}-\left(\boldsymbol{\sigma}_{h}^{n+1}\right)^{-1}\right)+\frac{1}{\mathrm{Wi}}\left(\boldsymbol{\sigma}_{h}^{n+1}-\boldsymbol{I}\right):\left(\boldsymbol{I}-\left(\boldsymbol{\sigma}_{h}^{n+1}\right)^{-1}\right)\right] .
\end{aligned}
$$

We first examine the terms associated with momentum conservation and incompressibility. We recall that $\boldsymbol{u}_{h}^{n+1}$ satisfies (3.3) since we use Scott-Vogelius finite elements. By the Stokes theorem (using the no-slip boundary condition), we immediately obtain:

$$
\int_{\mathcal{D}} \boldsymbol{u}_{h}^{n} \cdot \nabla\left|\boldsymbol{u}_{h}^{n+1}\right|^{2}=-\int_{\mathcal{D}}\left(\operatorname{div} \boldsymbol{u}_{h}^{n}\right)\left|\boldsymbol{u}_{h}^{n+1}\right|^{2}=0 .
$$

The terms involving $p_{h}^{n+1}$ also cancel. We now consider the terms involving $\boldsymbol{\sigma}_{h}^{n+1}$. The upper-convective term in the tensor derivative rewrites:

$$
\left(\boldsymbol{\nabla} \boldsymbol{u}_{h}^{n+1}\right) \boldsymbol{\sigma}_{h}^{n+1}:\left(\boldsymbol{I}-\left(\boldsymbol{\sigma}_{h}^{n+1}\right)^{-1}\right)=\boldsymbol{\sigma}_{h}^{n+1}: \nabla \boldsymbol{u}_{h}^{n+1}-\operatorname{div} \boldsymbol{u}_{h}^{n+1},
$$

which vanishes after combination with the extra-stress term $\boldsymbol{\sigma}_{h}^{n+1}: \boldsymbol{\nabla} \boldsymbol{u}_{h}^{n+1}$ in the momentum conservation equation, and using the incompressibility property. The last term rewrites:

$$
\left(\boldsymbol{\sigma}_{h}^{n+1}-\boldsymbol{I}\right):\left(\boldsymbol{I}-\left(\boldsymbol{\sigma}_{h}^{n+1}\right)^{-1}\right)=\operatorname{tr}\left(\boldsymbol{\sigma}_{h}^{n+1}+\left(\boldsymbol{\sigma}_{h}^{n+1}\right)^{-1}-2 \boldsymbol{I}\right) .
$$

The remaining term writes:

$$
\begin{aligned}
\int_{\mathcal{D}}\left(\boldsymbol{\sigma}_{h}^{n+1}-\boldsymbol{\sigma}_{h}^{n} \circ X^{n}\left(t^{n}\right)\right):\left(\boldsymbol{I}-\left(\boldsymbol{\sigma}_{h}^{n+1}\right)^{-1}\right)=\int_{\mathcal{D}} & \operatorname{tr}\left(\boldsymbol{\sigma}_{h}^{n+1}\right)-\operatorname{tr}\left(\boldsymbol{\sigma}_{h}^{n} \circ X^{n}\left(t^{n}\right)\right) \\
& +\operatorname{tr}\left(\left[\boldsymbol{\sigma}_{h}^{n} \circ X^{n}\left(t^{n}\right)\right]\left[\boldsymbol{\sigma}_{h}^{n+1}\right]^{-1}-\boldsymbol{I}\right) .
\end{aligned}
$$

We first make use of (1.10) with $\boldsymbol{\sigma}=\boldsymbol{\sigma}_{h}^{n} \circ X^{n}\left(t^{n}\right)$ and $\boldsymbol{\tau}=\boldsymbol{\sigma}_{h}^{n+1}$ :

$$
\operatorname{tr}\left(\left[\boldsymbol{\sigma}_{h}^{n} \circ X^{n}\left(t^{n}\right)\right]\left[\boldsymbol{\sigma}_{h}^{n+1}\right]^{-1}-\boldsymbol{I}\right) \geq \operatorname{tr} \ln \left(\boldsymbol{\sigma}_{h}^{n} \circ X^{n}\left(t^{n}\right)\right)-\operatorname{tr} \ln \left(\boldsymbol{\sigma}_{h}^{n+1}\right) .
$$

Then, we have:

$$
\int_{\mathcal{D}}-\operatorname{tr}\left(\boldsymbol{\sigma}_{h}^{n} \circ X^{n}\left(t^{n}\right)+\ln \left(\boldsymbol{\sigma}_{h}^{n} \circ X^{n}\left(t^{n}\right)\right)\right)=\int_{\mathcal{D}}-\operatorname{tr}\left(\boldsymbol{\sigma}_{h}^{n}+\ln \boldsymbol{\sigma}_{h}^{n}\right),
$$

since the strong incompressibility property $\left(\operatorname{div} \boldsymbol{u}_{h}^{n}=0\right)$ implies that the flow $X^{n}(t)$ defines a mapping with constant Jacobian equal to 1 for all $t \in\left[t^{n}, t^{n+1}\right]$. Finally, we get the following lower bound:

$$
\int_{\mathcal{D}}\left(\boldsymbol{\sigma}_{h}^{n+1}-\boldsymbol{\sigma}_{h}^{n} \circ X^{n}\left(t^{n}\right)\right):\left(\boldsymbol{I}-\left(\boldsymbol{\sigma}_{h}^{n+1}\right)^{-1}\right) \geq \int_{\mathcal{D}} \operatorname{tr}\left(\boldsymbol{\sigma}_{h}^{n+1}-\ln \boldsymbol{\sigma}_{h}^{n+1}\right)-\operatorname{tr}\left(\boldsymbol{\sigma}_{h}^{n}-\ln \boldsymbol{\sigma}_{h}^{n}\right),
$$

hence the result (4.2). 
Notice that $\operatorname{tr}\left(\boldsymbol{\sigma}_{h}^{n+1}+\left(\boldsymbol{\sigma}_{h}^{n+1}\right)^{-1}-2 \boldsymbol{I}\right) \geq 0$ by virtue of the equation (1.8), which shows that the sequence $\left(F_{h}^{n}\right)_{0 \leq n \leq N_{T}}$ is non-increasing.

\subsubsection{The discontinuous Galerkin method}

Proposition 4.2. Let $\left(\boldsymbol{u}_{h}^{n}, p_{h}^{n}, \boldsymbol{\sigma}_{h}^{n}\right)_{0 \leq n \leq N_{T}}$ be a solution to (3.8), such that $\boldsymbol{\sigma}_{h}^{n}$ is positive definite. Then, the free energy $F_{h}^{n}$ defined by (4.1) satisfies the free energy estimate (4.2). In particular, the sequence $\left(F_{h}^{n}\right)_{0 \leq n \leq N_{T}}$ is non-increasing.

Proof of Proposition 4.2. We only point out the differences with the proof of Proposition 4.1. They consist in the treatment of the discretization of the advection terms for $\boldsymbol{\sigma}_{h}$. We recall that the test function in stress is $\phi=\frac{\varepsilon}{2 \mathrm{Wi}}\left(\boldsymbol{I}-\left(\boldsymbol{\sigma}_{h}^{n+1}\right)^{-1}\right)$, so that we have:

$$
\begin{aligned}
& \sum_{j=1}^{N_{E}} \int_{E_{j}}\left|\boldsymbol{u}_{h}^{n} \cdot \boldsymbol{n}\right| \llbracket \boldsymbol{\sigma}_{h}^{n+1} \rrbracket:\left(\boldsymbol{I}-\left(\boldsymbol{\sigma}_{h}^{n+1}\right)^{-1}\right)^{+}=\sum_{j=1}^{N_{E}} \int_{E_{j}}\left|\boldsymbol{u}_{h}^{n} \cdot \boldsymbol{n}\right| \llbracket \operatorname{tr}\left(\boldsymbol{\sigma}_{h}^{n+1}\right) \rrbracket \\
&+\left|\boldsymbol{u}_{h}^{n} \cdot \boldsymbol{n}\right| \operatorname{tr}\left(\boldsymbol{\sigma}_{h}^{n+1,-}\left(\boldsymbol{\sigma}_{h}^{n+1,+}\right)^{-1}-\boldsymbol{I}\right) .
\end{aligned}
$$

Again, we make use of (1.10), with $\boldsymbol{\sigma}=\boldsymbol{\sigma}_{h}^{n+1,-}$ and $\boldsymbol{\tau}=\boldsymbol{\sigma}_{h}^{n+1,+}$ :

$$
\operatorname{tr}\left(\boldsymbol{\sigma}_{h}^{n+1,-}\left(\boldsymbol{\sigma}_{h}^{n+1,+}\right)^{-1}-\boldsymbol{I}\right) \geq \operatorname{tr}\left(\ln \boldsymbol{\sigma}_{h}^{n+1,-}-\ln \boldsymbol{\sigma}_{h}^{n+1,+}\right) .
$$

We get, by formula (3.5), the fact that $\boldsymbol{\sigma}_{h}^{n+1} \in\left(\mathbb{P}_{0}\right)^{\frac{d(d+1)}{2}}$, the Stokes theorem and the incompressibility property (3.3):

$$
\begin{aligned}
\sum_{j=1}^{N_{E}} \int_{E_{j}}\left|\boldsymbol{u}_{h}^{n} \cdot \boldsymbol{n}\right| \llbracket \boldsymbol{\sigma}_{h}^{n+1} \rrbracket:\left(\boldsymbol{I}-\left(\boldsymbol{\sigma}_{h}^{n+1}\right)^{-1}\right)^{+} & \geq \sum_{j=1}^{N_{E}} \int_{E_{j}}\left|\boldsymbol{u}_{h}^{n} \cdot \boldsymbol{n}\right| \llbracket \operatorname{tr}\left(\boldsymbol{\sigma}_{h}^{n+1}-\ln \boldsymbol{\sigma}_{h}^{n+1}\right) \rrbracket \\
& =-\sum_{k=1}^{N_{K}} \int_{\partial K_{k}}\left(\boldsymbol{u}_{h}^{n} \cdot \boldsymbol{n}_{K_{k}}\right) \operatorname{tr}\left(\boldsymbol{\sigma}_{h}^{n+1}-\ln \boldsymbol{\sigma}_{h}^{n+1}\right) \\
& =-\left.\sum_{k=1}^{N_{K}}\left(\operatorname{tr}\left(\boldsymbol{\sigma}_{h}^{n+1}-\ln \boldsymbol{\sigma}_{h}^{n+1}\right)\right)\right|_{K_{k}} \int_{\partial K_{k}} \boldsymbol{u}_{h}^{n} \cdot \boldsymbol{n}_{K_{k}} \\
& =-\left.\sum_{k=1}^{N_{K}}\left(\operatorname{tr}\left(\boldsymbol{\sigma}_{h}^{n+1}-\ln \boldsymbol{\sigma}_{h}^{n+1}\right)\right)\right|_{K_{k}} \int_{K_{k}} \operatorname{div}\left(\boldsymbol{u}_{h}^{n}\right) \\
& =0 .
\end{aligned}
$$

Moreover, it is easy to prove the following, using the same technique as in the proof of Proposition 4.1:

$$
\int_{\mathcal{D}}\left(\boldsymbol{\sigma}_{h}^{n+1}-\boldsymbol{\sigma}_{h}^{n}\right):\left(\boldsymbol{I}-\left(\boldsymbol{\sigma}_{h}^{n+1}\right)^{-1}\right) \geq \int_{\mathcal{D}} \operatorname{tr}\left(\boldsymbol{\sigma}_{h}^{n+1}-\ln \boldsymbol{\sigma}_{h}^{n+1}\right)-\operatorname{tr}\left(\boldsymbol{\sigma}_{h}^{n}-\ln \boldsymbol{\sigma}_{h}^{n}\right) .
$$

This concludes the proof.

\subsection{Free energy estimates with piecewise constant discretization of $\psi_{h}$}

This section is the equivalent of the previous section for the log-formulation. 


\subsubsection{The characteristic method}

Proposition 4.3. Let $\left(\boldsymbol{u}_{h}^{n}, p_{h}^{n}, \boldsymbol{\psi}_{h}^{n}\right)_{0 \leq n \leq N_{T}}$ be a solution to (3.10). Then, the free energy of the solution $\left(\boldsymbol{u}_{h}^{n}, p_{h}^{n}, \boldsymbol{\psi}_{h}^{n}\right)$ :

satisfies:

$$
F_{h}^{n}=F\left(\boldsymbol{u}_{h}^{n}, \mathrm{e}^{\boldsymbol{\psi}_{h}^{n}}\right)=\frac{\operatorname{Re}}{2} \int_{\mathcal{D}}\left|\boldsymbol{u}_{h}^{n}\right|^{2}+\frac{\varepsilon}{2 \mathrm{Wi}} \int_{\mathcal{D}} \operatorname{tr}\left(\mathrm{e}^{\boldsymbol{\psi}_{h}^{n}}-\boldsymbol{\psi}_{h}^{n}-\boldsymbol{I}\right)
$$

$$
F_{h}^{n+1}-F_{h}^{n}+\int_{\mathcal{D}} \frac{\mathrm{Re}}{2}\left|\boldsymbol{u}_{h}^{n+1}-\boldsymbol{u}_{h}^{n}\right|^{2}+\Delta t \int_{\mathcal{D}}(1-\varepsilon)\left|\nabla \boldsymbol{u}_{h}^{n+1}\right|^{2}+\frac{\varepsilon}{2 \mathrm{Wi}^{2}} \operatorname{tr}\left(\mathrm{e}^{\boldsymbol{\psi}_{h}^{n+1}}+\mathrm{e}^{-\boldsymbol{\psi}_{h}^{n+1}}-2 I\right) \leq 0 .
$$

In particular, the sequence $\left(F_{h}^{n}\right)_{0 \leq n \leq N_{T}}$ is non-increasing.

Proof of Proposition 4.3. We shall use as test functions $\left(\boldsymbol{u}_{h}^{n+1}, p_{h}^{n+1}, \frac{\varepsilon}{2 \mathrm{Wi}}\left(\mathrm{e}^{\boldsymbol{\psi}_{h}^{n+1}}-\boldsymbol{I}\right)\right)$ in (3.10). We emphasize that, as long as the solution $\left(\boldsymbol{u}_{h}^{n+1}, p_{h}^{n+1}, \boldsymbol{\psi}_{h}^{n+1}\right)$ exists (see Prop. 3.6), $\mathrm{e}_{h}^{\boldsymbol{\psi}_{h}^{n+1}}$ is well-defined, symmetric positive definite and piecewise constant.

The terms are treated similarly as in the proof of Proposition 4.1. For the material derivative of $\boldsymbol{\psi}_{h}$, we have:

$$
\int_{\mathcal{D}}\left(\boldsymbol{\psi}_{h}^{n+1}-\boldsymbol{\psi}_{h}^{n} \circ X^{n}\left(t^{n}\right)\right):\left(\mathrm{e}^{\boldsymbol{\psi}_{h}^{n+1}}-\boldsymbol{I}\right)=\int_{\mathcal{D}}\left(\boldsymbol{\psi}_{h}^{n+1}-\boldsymbol{\psi}_{h}^{n} \circ X^{n}\left(t^{n}\right)\right): \mathrm{e}^{\boldsymbol{\psi}_{h}^{n+1}}-\operatorname{tr}\left(\boldsymbol{\psi}_{h}^{n+1}-\boldsymbol{\psi}_{h}^{n} \circ X^{n}\left(t^{n}\right)\right) .
$$

Using the equation (1.11) with $\boldsymbol{\sigma}=\mathrm{e}^{\boldsymbol{\psi}_{h}^{n+1}}$ and $\boldsymbol{\tau}=\mathrm{e}^{\boldsymbol{\psi}_{h}^{n} \circ X^{n}\left(t^{n}\right)}$, we obtain:

$$
\left(\boldsymbol{\psi}_{h}^{n+1}-\boldsymbol{\psi}_{h}^{n} \circ X^{n}\left(t^{n}\right)\right): \mathrm{e}^{\boldsymbol{\psi}_{h}^{n+1}} \geq \operatorname{tr}\left(\mathrm{e}^{\boldsymbol{\psi}_{h}^{n+1}}-\mathrm{e}^{\boldsymbol{\psi}_{h}^{n} \circ X^{n}\left(t^{n}\right)}\right)
$$

and thus:

$$
\begin{aligned}
\int_{\mathcal{D}}\left(\boldsymbol{\psi}_{h}^{n+1}-\boldsymbol{\psi}_{h}^{n} \circ X^{n}\left(t^{n}\right)\right):\left(\mathrm{e}^{\boldsymbol{\psi}_{h}^{n+1}}-\boldsymbol{I}\right) & \geq \int_{\mathcal{D}} \operatorname{tr}\left(\mathrm{e}^{\boldsymbol{\psi}_{h}^{n+1}}-\boldsymbol{\psi}_{h}^{n+1}\right)-\int_{\mathcal{D}} \operatorname{tr}\left(\mathrm{e}^{\boldsymbol{\psi}_{h}^{n}}-\boldsymbol{\psi}_{h}^{n}\right) \circ X^{n}\left(t^{n}\right) \\
& =\int_{\mathcal{D}} \operatorname{tr}\left(\mathrm{e}^{\boldsymbol{\psi}_{h}^{n+1}}-\boldsymbol{\psi}_{h}^{n+1}\right)-\int_{\mathcal{D}} \operatorname{tr}\left(\mathrm{e}^{\boldsymbol{\psi}_{h}^{n}}-\boldsymbol{\psi}_{h}^{n}\right),
\end{aligned}
$$

where the fact that the Jacobian of the flow $X^{n}$ is constant equal to one (because $\boldsymbol{u}_{h}^{n}$ is divergence-free) has been used in the change of variable in the last equality.

Besides, using the equation (2.16), we have:

$$
\int_{\mathcal{D}}\left(\boldsymbol{\Omega}_{h}^{n+1} \boldsymbol{\psi}_{h}^{n+1}-\boldsymbol{\psi}_{h}^{n+1} \boldsymbol{\Omega}_{h}^{n+1}\right):\left(\mathrm{e}^{\boldsymbol{\psi}_{h}^{n+1}}-\boldsymbol{I}\right)=\int_{\mathcal{D}}\left(\boldsymbol{\Omega}_{h}^{n+1} \boldsymbol{\psi}_{h}^{n+1}-\boldsymbol{\psi}_{h}^{n+1} \boldsymbol{\Omega}_{h}^{n+1}\right): \mathrm{e}^{\boldsymbol{\psi}_{h}^{n+1}}=0 .
$$

Last, using (2.15):

$$
\begin{aligned}
\int_{\mathcal{D}} \boldsymbol{B}_{h}^{n+1}:\left(\mathrm{e}^{\boldsymbol{\psi}_{h}^{n+1}}-\boldsymbol{I}\right) & =\int_{\mathcal{D}} \boldsymbol{B}_{h}^{n+1}: \mathrm{e}^{\boldsymbol{\psi}_{h}^{n+1}}-\int_{\mathcal{D}} \operatorname{tr}\left(\boldsymbol{B}_{h}^{n+1}\right) \\
& =\int_{\mathcal{D}} \boldsymbol{\nabla} \boldsymbol{u}_{h}^{n+1}: \mathrm{e}^{\boldsymbol{\psi}_{h}^{n+1}}-\int_{\mathcal{D}} \operatorname{div}\left(\boldsymbol{u}_{h}^{n+1}\right)=\int_{\mathcal{D}} \boldsymbol{\nabla} \boldsymbol{u}_{h}^{n+1}: \mathrm{e}^{\boldsymbol{\psi}_{h}^{n+1}}
\end{aligned}
$$

which cancels out with the same term $\int_{\mathcal{D}} \mathrm{e}^{\boldsymbol{\psi}_{h}^{n+1}}: \boldsymbol{\nabla} \boldsymbol{u}_{h}^{n+1}$ in the momentum equation.

\subsubsection{The discontinuous Galerkin method}

Proposition 4.4. Let $\left(\boldsymbol{u}_{h}^{n}, p_{h}^{n}, \boldsymbol{\psi}_{h}^{n}\right)_{0 \leq n \leq N_{T}}$ be a solution to (3.11). Then, the free energy $F_{h}^{n}$ defined by (4.4) satisfies the free energy estimate (4.5). In particular, the sequence $\left(F_{h}^{n}\right)_{0 \leq n \leq N_{T}}$ is non-increasing.

The proof is straightforward using elements of the proofs of Propositions 4.3 and 4.2. 
TABLE 1. Summary of the arguments with $\left(\boldsymbol{u}_{h}, p_{h}, \boldsymbol{\sigma}_{h}\right)$ or $\left(\boldsymbol{u}_{h}, p_{h}, \boldsymbol{\psi}_{h}\right)$ in $\left(\mathbb{P}_{2}\right)^{d} \times \mathbb{P}_{1, \mathrm{disc}} \times\left(\mathbb{P}_{0}\right)^{\frac{d(d+1)}{2}}$.

\begin{tabular}{|l||l|l|}
\hline Advection discretized by & \multicolumn{1}{|c|}{ Characteristics } & \multicolumn{1}{c|}{ DG } \\
\hline Requirements for $\boldsymbol{u}_{h}$ & $\operatorname{div} \boldsymbol{u}_{h}=0$ & $\int_{\mathcal{D}} q \operatorname{div} \boldsymbol{u}_{h}=0, \forall q \in \mathbb{P}_{0}$ \\
& $\left(\Rightarrow \operatorname{det}\left(\nabla_{\boldsymbol{x}} X^{n}\right) \equiv 1\right)$ & and \\
& $\left(\left.\Rightarrow\left(\boldsymbol{u}_{h} \cdot \boldsymbol{n}\right)\right|_{E_{j}}\right.$ well defined $)$ & $\left.\left(\boldsymbol{u}_{h} \cdot \boldsymbol{n}\right)\right|_{E_{j}}$ well defined \\
\hline
\end{tabular}

\subsection{Other finite elements for $\left(u_{h}, p_{h}\right)$}

In this section, we review some finite element spaces for $\left(\boldsymbol{u}_{h}, p_{h}\right)$ other than Scott-Vogelius for which the results of the last two sections still hold.

First, let us stress the key arguments we used in the proofs above. If the advection terms $\boldsymbol{u} \cdot \boldsymbol{\nabla} \boldsymbol{\sigma}$ and $\boldsymbol{u} \cdot \boldsymbol{\nabla} \boldsymbol{\psi}$ are discretized by the characteristic method, we need the velocity field $\boldsymbol{u}_{h}^{n}$ to be divergence-free:

$$
\operatorname{div} \boldsymbol{u}_{h}^{n}=0,
$$

in order for the flow $X^{n}$ satisfying (3.7) to be with Jacobian one. When $\boldsymbol{u}_{h}^{n}$ is only piecewise smooth (consider below the case of $\mathbb{P}_{1, \text { disc }}$ velocity fields), the divergence in the left-hand side of (4.6) should be understood in the sense of distributions. By the way, the equation (4.6) ensures that the trace of the normal component $\boldsymbol{u}_{h}^{n} \cdot \boldsymbol{n}$ on the edges of the mesh is uniquely defined, which is a sufficient condition to define the flow associated with an elementwise-Lipschitz-continuous vector field $\boldsymbol{u}_{h}^{n}$ through (3.7), and which is necessary to treat the advection term in the Navier-Stokes equation (see [40]).

If the advection terms are discretized by the discontinuous Galerkin method, it is necessary that the trace of the normal component of $\boldsymbol{u}_{h}$ be uniquely defined on the edges of the mesh since it appears in the jump terms $\sum_{j=1}^{N_{E}} \int_{E_{j}}\left|\boldsymbol{u}_{h}^{n} \cdot \boldsymbol{n}\right| \llbracket \boldsymbol{\sigma}_{h}^{n+1} \rrbracket: \boldsymbol{\phi}^{+}$or $\sum_{j=1}^{N_{E}} \int_{E_{j}}\left|\boldsymbol{u}_{h}^{n} \cdot \boldsymbol{n}\right| \llbracket \boldsymbol{\psi}_{h}^{n+1} \rrbracket: \boldsymbol{\phi}^{+}$in the variational formulations. But to obtain (4.3), and contrary to the characteristic method, only the following weak incompressibility property is needed:

which is equivalent to writing:

$$
\forall k=1, \ldots, N_{K}, \quad \int_{K_{k}} \operatorname{div} \boldsymbol{u}_{h}^{n}=0,
$$

$$
\forall q \in \mathbb{P}_{0}, \quad \int_{\mathcal{D}} \operatorname{div}\left(\boldsymbol{u}_{h}^{n}\right) q=0 .
$$

The properties needed to obtain the discrete free energy estimates are summarized in Table 1.

Below, we consider the following alternative choices of the finite elements space for $\left(\boldsymbol{u}_{h}, p_{h}\right)$ :

- the Taylor-Hood finite element space: $\left(\boldsymbol{u}_{h}, p_{h}\right) \in\left(\mathbb{P}_{2}\right)^{d} \times \mathbb{P}_{1}$, which satisfies the Babuška-Brezzi inf-sup condition, whatever the mesh;

- the mixed Crouzeix-Raviart finite element space (see [12]): $\left(\boldsymbol{u}_{h}, p_{h}\right) \in\left(\mathbb{P}_{1}^{C R}\right)^{d} \times \mathbb{P}_{0}$, where

$$
\mathbb{P}_{1}^{C R}=\left\{v \in \mathbb{P}_{1, \mathrm{disc}} \mid \forall E_{j}, \int_{E_{j}} \llbracket v \rrbracket=0\right\},
$$

which also satisfies the Babuška-Brezzi inf-sup condition, whatever the mesh;

- stabilized formulations for $\left(\boldsymbol{u}_{h}, p_{h}\right) \in\left(\mathbb{P}_{1}\right)^{d} \times \mathbb{P}_{1}$ or $\left(\boldsymbol{u}_{h}, p_{h}\right) \in\left(\mathbb{P}_{1}\right)^{d} \times \mathbb{P}_{0}$.

This is not exhaustive, but it is sufficient to highlight which modifications are needed in the variational formulations, compared to the Scott-Vogelius mixed finite element, for the discrete free energy estimates to hold. In particular, some projection of the velocity field is needed in the discretization of the advection terms $\boldsymbol{u} \cdot \boldsymbol{\nabla} \boldsymbol{\sigma}$ and $\boldsymbol{u} \cdot \boldsymbol{\nabla} \boldsymbol{\psi}$ in order to satisfy the requirements of Table 1 . These projection operators are introduced in the next Section 4.3.1. The results of Section 4.3 are summarized in Table 2. 
Remark 4.5. For all the finite element spaces introduced here, similar existence results as those stated in Section 3.5 hold. For the sake of conciseness, we do not restate these results, but rather concentrate on establishing the free energy estimates.

\subsubsection{Some useful projection operators for the velocity field}

Let us introduce three projection operators for the velocity field.

Following [40], we first define the orthogonal projection $P_{h}^{\text {rot }}$ onto the piecewise constant solenoidal vector fields built from affine continuous scalar fields:

$$
\left\{\nabla \times \zeta_{h} \mid \zeta_{h} \in\left(\mathbb{P}_{1}\right)^{d}, \zeta_{h} \times \boldsymbol{n}=0 \text { on } \partial \mathcal{D}\right\}
$$

We suppose here that $d=3$, but the extension to the case $d=2$ is straightforward. We set $P_{h}^{\text {rot }}\left(\boldsymbol{u}_{h}\right)=\nabla \times \psi_{h}$ where $\psi_{h} \in\left(\mathbb{P}_{1}\right)^{d}$, such that $\psi_{h} \times\left.\boldsymbol{n}\right|_{\partial \mathcal{D}}=0$, satisfies:

$$
\int_{\mathcal{D}}\left(\nabla \psi_{h}\right):\left(\nabla \zeta_{h}\right)=\int_{\mathcal{D}} \boldsymbol{u}_{h} \cdot\left(\nabla \times \zeta_{h}\right), \forall \zeta_{h} \in\left(\mathbb{P}_{1}\right)^{d}, \zeta_{h} \times\left.\boldsymbol{n}\right|_{\partial \mathcal{D}}=0 .
$$

Because $P_{h}^{\text {rot }}\left(\boldsymbol{u}_{h}\right)$ is solenoidal, we always have the strong incompressibility property (4.6):

$$
\operatorname{div} P_{h}^{\text {rot }}\left(\boldsymbol{u}_{h}\right)=0
$$

for any velocity field $\boldsymbol{u}_{h}$. Of course, this operator is consistent only for divergence-free velocity fields $\boldsymbol{u}_{h}$ (or velocity field $\boldsymbol{u}_{h}$ with vanishing divergence when $h$ goes to zero). See [40] for consistency results.

Second, following [13], we define the Raviart-Thomas interpolator $P_{h}^{R T_{0}}$ onto the vector subspace of $\left(\mathbb{P}_{1, \text { disc }}\right)^{d}$ made of the vector fields in $\left(\mathbb{P}_{0}\right)^{d}+\boldsymbol{x} \mathbb{P}_{0}$ with continuous normal component across the edges $E_{j}$ (whose trace on $E_{j}$ is then uniquely defined). The projection $P_{h}^{R T_{0}}\left(\boldsymbol{u}_{h}^{n}\right)$ clearly satisfies, for any element $K_{k}$ :

$$
\int_{K_{k}} \operatorname{div} \boldsymbol{u}_{h}=\int_{\partial K_{k}} \boldsymbol{u}_{h}^{n} \cdot \boldsymbol{n}_{K_{k}}=\int_{\partial K_{k}} P_{h}^{R T_{0}}\left(\boldsymbol{u}_{h}^{n}\right) \cdot \boldsymbol{n}_{K_{k}}=\int_{K_{k}} \operatorname{div} P_{h}^{R T_{0}}\left(\boldsymbol{u}_{h}^{n}\right) .
$$

Thus, it satisfies the weak incompressibility property (4.7):

$$
\forall q \in \mathbb{P}_{0}, \int_{\mathcal{D}} \operatorname{div}\left(P_{h}^{R T_{0}}\left(\boldsymbol{u}_{h}^{n}\right)\right) q=0
$$

if, and only if, the velocity field $\boldsymbol{u}_{h}^{n}$ also satisfies it.

Third, we define $P_{h}^{B D M}$ as the Brezzi-Douglas-Marini projection operator [9,10]. It is with value in $\left(\mathbb{P}_{1}\right)^{d}$. This projection operator satisfies the same divergence preservation property (4.9) than $P_{h}^{R T_{0}}$, but is of better accuracy.

Note that $P_{h}^{B D M}$ and $P_{h}^{R T_{0}}$ are local interpolating operators in the sense that all the computations can be made elementwise. This is not the case for $P_{h}^{\text {rot }}$. In addition, we will need the following lemma:

Lemma 4.6. For any velocity field $\boldsymbol{u}_{h}^{n}$ such that the previously defined interpolating operators are well defined, the normal components of the interpolated vector field, $P_{h}^{\operatorname{rot}}\left(\boldsymbol{u}_{h}^{n}\right) \cdot \boldsymbol{n}, P_{h}^{R T_{0}}\left(\boldsymbol{u}_{h}^{n}\right) \cdot \boldsymbol{n}$ and $P_{h}^{B D M}\left(\boldsymbol{u}_{h}^{n}\right) \cdot \boldsymbol{n}$ are also well defined on any internal edges $E_{j}$. Moreover, if $\boldsymbol{u}_{h}^{n} \in\left(\mathbb{P}_{1, \text { disc }}\right)^{d}$ is a velocity field such that, for all $k=1, \ldots, N_{K}$ :

$$
\int_{K_{k}} \operatorname{div}\left(\boldsymbol{u}_{h}^{n}\right)=0
$$

then $\operatorname{div}\left(P_{h}^{R T_{0}}\left(\boldsymbol{u}_{h}^{n}\right)\right)=\operatorname{div}\left(P_{h}^{B D M}\left(\boldsymbol{u}_{h}^{n}\right)\right)=0$ (in the sense of distributions). 
Proof. By construction, $P_{h}^{R T_{0}}$ and $P_{h}^{B D M}$ take their values in the set of velocity fields whose normal components are continuous across the edges. This is also the case for $P_{h}^{\text {rot }}$ since $P_{h}^{\text {rot }}$ takes its value in the set of divergencefree velocity fields. Then, from the equation (4.9), we have $\int_{K_{k}} \operatorname{div}\left(P_{h}^{R T_{0}}\left(\boldsymbol{u}_{h}^{n}\right)\right)=0$. $\operatorname{Since} \operatorname{div}\left(P_{h}^{R T_{0}}\left(\boldsymbol{u}_{h}^{n}\right)\right)$ is in $\left(\mathbb{P}_{0}\right)^{d}$, this shows that $\operatorname{div}\left(P_{h}^{R T_{0}}\left(\boldsymbol{u}_{h}^{n}\right)\right)$ is zero in any element $K_{k}$. Finally, $P_{h}^{R T_{0}}\left(\boldsymbol{u}_{h}^{n}\right)$ has continuous normal components across the edges of the mesh. This shows that $\operatorname{div}\left(P_{h}^{R T_{0}}\left(\boldsymbol{u}_{h}^{n}\right)\right)=0$ in the sense of distributions. The same proof holds for the projection operator $P_{h}^{B D M}$.

\subsubsection{Alternative mixed finite element space for $\left(\boldsymbol{u}_{h}, p_{h}\right)$ with inf-sup condition}

In this section, we show how to derive discrete free energy estimates with mixed finite element spaces for the velocity and pressure fields which satisfy the inf-sup condition, but which are not the Scott-Vogelius finite elements.

Let us first consider the Taylor-Hood element for $\left(\boldsymbol{u}_{h}, p_{h}\right)$, that is $\left(\mathbb{P}_{2}\right)^{d} \times \mathbb{P}_{1}$. In this case, since the velocity field $\boldsymbol{u}_{h}$ is not divergence-free either in the weak form (4.7), or in the strong form (4.6), a projection of the velocity field is required in the discretization of the advection terms $\boldsymbol{u} \cdot \nabla \boldsymbol{\sigma}$ and $\boldsymbol{u} \cdot \boldsymbol{\nabla} \boldsymbol{\psi}$. More precisely, we need to use the projection velocity $P_{h}^{\text {rot }} \boldsymbol{u}_{h}^{n}$ (and, among the three projection operators we introduced above, this is the only one which is such that the strong or weak incompressibility is satisfied). For the characteristic method, one uses the flow $X^{n}(t)$ satisfying:

$$
\left\{\begin{array}{l}
\frac{\mathrm{d}}{\mathrm{d} t} X^{n}(t, x)=P_{h}^{\mathrm{rot}} \boldsymbol{u}_{h}^{n}\left(X^{n}(t, x)\right), \quad \forall t \in\left[t^{n}, t^{n+1}\right], \\
X^{n}\left(t^{n+1}, x\right)=x
\end{array}\right.
$$

For the discontinuous Galerkin method, the advection term in the conformation-tensor formulations writes (see the last line in (3.8)):

$$
+\sum_{j=1}^{N_{E}} \int_{E_{j}}\left|P_{h}^{\mathrm{rot}}\left(\boldsymbol{u}_{h}^{n}\right) \cdot \boldsymbol{n}\right| \llbracket \boldsymbol{\sigma}_{h}^{n+1} \rrbracket: \boldsymbol{\phi}^{+}
$$

Notice that in the terms $\llbracket \boldsymbol{\sigma}_{h}^{n+1} \rrbracket: \boldsymbol{\phi}^{+}$, the projected velocity $P_{h}^{\text {rot }} \boldsymbol{u}_{h}^{n}$ is used to define the upstream and downstream values following (3.4). Another modification, which is specific to the Navier-Stokes equation, is needed to treat the advection term on the velocity. Namely, one needs to add to the weak formulation the so-called Temam correction term (see [45]):

$$
+\frac{\operatorname{Re}}{2} \int_{\mathcal{D}} \operatorname{div}\left(\boldsymbol{u}_{h}^{n}\right)\left(\boldsymbol{v} \cdot \boldsymbol{u}_{h}^{n+1}\right)
$$

in such a way that, when $\boldsymbol{u}_{h}^{n+1}$ is used as a test function:

$$
\operatorname{Re} \int_{\mathcal{D}}\left(\boldsymbol{u}_{h}^{n} \cdot \nabla \boldsymbol{u}_{h}^{n+1}\right) \cdot \boldsymbol{u}_{h}^{n+1}+\frac{\operatorname{Re}}{2} \int_{\mathcal{D}} \operatorname{div}\left(\boldsymbol{u}_{h}^{n}\right)\left|\boldsymbol{u}_{h}^{n+1}\right|^{2}=0 .
$$

With these modifications (projection of the velocity field in the advection terms, and Temam correction term), the free energy estimate (4.2) is satisfied by the scheme. Similar results (discrete free energy estimates for $\left(\boldsymbol{u}_{h}, p_{h}, \boldsymbol{\psi}_{h}\right)$ in $\left.\left(\mathbb{P}_{2}\right)^{d} \times \mathbb{P}_{1} \times\left(\mathbb{P}_{0}\right)^{\frac{d(d+1)}{2}}\right)$ can be proved on the log-formulation.

Let us now discuss the use of Crouzeix-Raviart finite elements for velocity: $\left(\boldsymbol{u}_{h}, p_{h}, \boldsymbol{\sigma}_{h}\right)$ in $\left(\mathbb{P}_{1}^{C R}\right)^{d} \times \mathbb{P}_{0} \times$ $\left(\mathbb{P}_{0}\right)^{\frac{d(d+1)}{2}}($ see $(4.8))$. In this case, the Navier-Stokes equations can be discretized using a characteristic method:

$$
0=\sum_{k=1}^{N_{K}} \int_{K_{k}} \operatorname{Re}\left(\frac{\boldsymbol{u}_{h}^{n+1}-\boldsymbol{u}_{h}^{n} \circ X^{n}\left(t_{n}\right)}{\Delta t}\right) \cdot \boldsymbol{v}-p_{h}^{n+1} \operatorname{div} \boldsymbol{v}+q \operatorname{div} \boldsymbol{u}_{h}^{n+1}+(1-\varepsilon) \nabla \boldsymbol{u}_{h}^{n+1}: \nabla \boldsymbol{v}+\frac{\varepsilon}{\mathrm{Wi}} \boldsymbol{\sigma}_{h}^{n+1}: \boldsymbol{\nabla} \boldsymbol{v}
$$


where $X^{n}$ is obtained from the projected velocity field $P_{h} \boldsymbol{u}_{h}^{n}$ as:

$$
\left\{\begin{array}{l}
\frac{\mathrm{d}}{\mathrm{d} t} X^{n}(t)=P_{h} \boldsymbol{u}_{h}^{n}\left(X^{n}(t)\right), \quad \forall t \in\left[t^{n}, t^{n+1}\right] \\
X^{n}\left(t^{n+1}\right)=x
\end{array}\right.
$$

The projected velocity $P_{h} \boldsymbol{u}_{h}^{n}$ is defined using any of the three projectors presented above, that is $P_{h}^{\text {rot }} \boldsymbol{u}_{h}^{n}, P_{h}^{R T_{0}} \boldsymbol{u}_{h}^{n}$ or $P_{h}^{B D M} \boldsymbol{u}_{h}^{n}$. The Navier-Stokes equations can also be discretized using a discontinuous Galerkin formulation:

$$
\begin{aligned}
0=\sum_{k=1}^{N_{K}} \int_{K_{k}} \operatorname{Re}\left(\frac{\boldsymbol{u}_{h}^{n+1}-\boldsymbol{u}_{h}^{n}}{\Delta t}\right. & \left.+P_{h}\left(\boldsymbol{u}_{h}^{n}\right) \cdot \boldsymbol{\nabla} \boldsymbol{u}_{h}^{n+1}\right) \cdot \boldsymbol{v}+\operatorname{Re} \sum_{j=1}^{N_{E}} \int_{E_{j}}\left|P_{h}\left(\boldsymbol{u}_{h}^{n}\right) \cdot \boldsymbol{n}\right| \llbracket \boldsymbol{u}_{h}^{n+1} \rrbracket \cdot\{\boldsymbol{v}\} \\
& +\sum_{k=1}^{N_{K}} \int_{K_{k}}-p_{h}^{n+1} \operatorname{div} \boldsymbol{v}+q \operatorname{div} \boldsymbol{u}_{h}^{n+1}+(1-\varepsilon) \boldsymbol{\nabla} \boldsymbol{u}_{h}^{n+1}: \boldsymbol{\nabla} \boldsymbol{v}+\frac{\varepsilon}{\mathrm{Wi}} \boldsymbol{\sigma}_{h}^{n+1}: \boldsymbol{\nabla} \boldsymbol{v} .
\end{aligned}
$$

Here again, $P_{h} \boldsymbol{u}_{h}^{n}$ is any of the three projectors presented above. We would like to mention that we are not aware that the projector $P_{h}^{\text {rot }}$ has ever been used with discontinuous Galerkin methods, so that the consistency of the discontinuous Galerkin approach combined with this projector still needs to be investigated. Likewise, the advection term $\boldsymbol{u} \cdot \nabla \boldsymbol{\sigma}$ in the equation on the stress can be treated by the characteristic method or the discontinuous Galerkin method, as above for the advection term in the Navier-Stokes equations. Notice that whatever the projecting operator used, $\operatorname{div}\left(P_{h} \boldsymbol{u}_{h}^{n}\right)=0$ holds (see Lem. 4.6 above). With this property, it is easy to check that Propositions 4.1 and 4.2 still hold for this finite element. For example, the advection term in the Navier-Stokes equations is treated as follows (using the fact that $\operatorname{div}\left(P_{h}\left(\boldsymbol{u}_{h}^{n}\right)\right)=0$ and (3.5)):

$$
\begin{aligned}
\sum_{k=1}^{N_{K}} \int_{K_{k}} & \left(P_{h}\left(\boldsymbol{u}_{h}^{n}\right) \cdot \nabla \boldsymbol{u}_{h}^{n+1}\right) \cdot \boldsymbol{u}_{h}^{n+1}+\sum_{j=1}^{N_{E}} \int_{E_{j}}\left|P_{h}\left(\boldsymbol{u}_{h}^{n}\right) \cdot \boldsymbol{n}\right| \llbracket \boldsymbol{u}_{h}^{n+1} \rrbracket \cdot\left\{\boldsymbol{u}_{h}^{n+1}\right\} \\
& =\sum_{k=1}^{N_{K}} \int_{K_{k}} \operatorname{div}\left(P_{h}\left(\boldsymbol{u}_{h}^{n}\right) \frac{\left|\boldsymbol{u}_{h}^{n+1}\right|^{2}}{2}\right)+\sum_{j=1}^{N_{E}} \int_{E_{j}}\left|P_{h}\left(\boldsymbol{u}_{h}^{n}\right) \cdot \boldsymbol{n}\right| \frac{1}{2} \llbracket\left|\boldsymbol{u}_{h}^{n+1}\right|^{2} \rrbracket \\
& =\sum_{k=1}^{N_{K}} \int_{K_{k}} \operatorname{div}\left(P_{h}\left(\boldsymbol{u}_{h}^{n}\right) \frac{\left|\boldsymbol{u}_{h}^{n+1}\right|^{2}}{2}\right)-\sum_{k=1}^{N_{K}} \int_{\partial K_{k}}\left(P_{h}\left(\boldsymbol{u}_{h}^{n}\right) \cdot \boldsymbol{n}_{K_{k}}\right) \frac{\left|\boldsymbol{u}_{h}^{n+1}\right|^{2}}{2}=0 .
\end{aligned}
$$

Discrete free energy estimates for $\left(\boldsymbol{u}_{h}, p_{h}, \boldsymbol{\psi}_{h}\right)$ in $\left(\mathbb{P}_{1}^{C R}\right)^{d} \times \mathbb{P}_{0} \times\left(\mathbb{P}_{0}\right)^{\frac{d(d+1)}{2}}$ can be similarly proven on the log-formulation.

\subsubsection{Alternative mixed finite element space for $\left(\boldsymbol{u}_{h}, p_{h}\right)$ without inf-sup}

It is also possible to choose a mixed finite elements space for $\left(\boldsymbol{u}_{h}, p_{h}\right)$ that does not satisfy the Babuška-Brezzi inf-sup condition, like $\left(\mathbb{P}_{1}\right)^{d} \times \mathbb{P}_{0}$ or $\left(\mathbb{P}_{1}\right)^{d} \times \mathbb{P}_{1}$. The loss of stability due to the incompatibility of the spaces can then be alleviated through a stabilization procedure, like Streamline Upwind Petrov Galerkin, Galerkin Least Square or Subgrid Scale Method (see $[11,18,21]$ ). In the following, we consider very simple stabilization procedures, for which only one simple quadratic term is added to the variational finite element formulation in order to restore stability of the discrete numerical scheme. 
Let us first consider the mixed finite element space $\left(\mathbb{P}_{1}\right)^{d} \times \mathbb{P}_{0}$ for $\left(\boldsymbol{u}_{h}, p_{h}\right)$. If the term $\boldsymbol{u} \cdot \boldsymbol{\nabla} \boldsymbol{\sigma}$ is discretized with the characteristic method, the system then writes:

$$
\begin{array}{r}
0=\sum_{k=1}^{N_{K}} \int_{K_{k}} \operatorname{Re}\left(\frac{\boldsymbol{u}_{h}^{n+1}-\boldsymbol{u}_{h}^{n}}{\Delta t}+\boldsymbol{u}_{h}^{n} \cdot \boldsymbol{\nabla} \boldsymbol{u}_{h}^{n+1}\right) \cdot \boldsymbol{v}+\frac{\operatorname{Re}}{2} \operatorname{div} \boldsymbol{u}_{h}^{n}\left(\boldsymbol{v} \cdot \boldsymbol{u}_{h}^{n+1}\right)+(1-\varepsilon) \boldsymbol{\nabla} \boldsymbol{u}_{h}^{n+1}: \boldsymbol{\nabla} \boldsymbol{v}+\frac{\varepsilon}{\mathrm{Wi}} \boldsymbol{\sigma}_{h}^{n+1}: \boldsymbol{\nabla} \boldsymbol{v} \\
-p_{h}^{n+1} \operatorname{div} \boldsymbol{v}+q \operatorname{div} \boldsymbol{u}_{h}^{n+1}+\left(\frac{\boldsymbol{\sigma}_{h}^{n+1}-\boldsymbol{\sigma}_{h}^{n} \circ X^{n}\left(t^{n}\right)}{\Delta t}\right): \boldsymbol{\phi}-\left(\left(\boldsymbol{\nabla} \boldsymbol{u}_{h}^{n+1}\right) \boldsymbol{\sigma}_{h}^{n+1}+\boldsymbol{\sigma}_{h}^{n+1}\left(\boldsymbol{\nabla} \boldsymbol{u}_{h}^{n+1}\right)^{T}\right): \boldsymbol{\phi} \\
+\frac{1}{\mathrm{Wi}}\left(\boldsymbol{\sigma}_{h}^{n+1}-\boldsymbol{I}\right): \boldsymbol{\phi}+\sum_{j=1}^{N_{E}}\left|E_{j}\right| \int_{E_{j}} \llbracket p_{h} \rrbracket \llbracket q \rrbracket, \quad(4.15)
\end{array}
$$

with a flow $X^{n}$ computed with the projected field $P_{h}^{\text {rot }}\left(\boldsymbol{u}_{h}^{n}\right)$ through (4.10). The projection operator $P_{h}^{\text {rot }}$ is the only one we can use among the three projectors we introduced in Section 4.3 .1 because the weak incompressibility property (4.7) is not satisfied by $\boldsymbol{u}_{h}^{n}$.

The stabilization procedure used in (4.15) has been studied in [25]. Proposition 4.1 holds for system (4.15), its proof being similar to the case of Taylor-Hood finite element (see Sect. 4.3.2), since the additional term $\sum_{j=1}^{N_{E}}\left|E_{j}\right| \int_{E_{j}} \llbracket p_{h} \rrbracket \llbracket q \rrbracket$ is non negative with the test function used in the proof. All this also holds mutatis mutandis for discretization of the advection terms by a discontinuous Galerkin method, and for the log-formulation.

Let us finally consider the mixed finite elements space $\left(\mathbb{P}_{1}\right)^{d} \times \mathbb{P}_{1}$ for $\left(\boldsymbol{u}_{h}, p_{h}\right)$. If the term $\boldsymbol{u} \cdot \boldsymbol{\nabla} \boldsymbol{\sigma}$ is discretized with the characteristic method, the system then writes:

$$
\begin{array}{r}
0=\int_{\mathcal{D}} \operatorname{Re}\left(\frac{\boldsymbol{u}_{h}^{n+1}-\boldsymbol{u}_{h}^{n}}{\Delta t}+\boldsymbol{u}_{h}^{n} \cdot \boldsymbol{\nabla} \boldsymbol{u}_{h}^{n+1}\right) \cdot \boldsymbol{v}+\frac{\operatorname{Re}}{2} \operatorname{div} \boldsymbol{u}_{h}^{n}\left(\boldsymbol{v} \cdot \boldsymbol{u}_{h}^{n+1}\right)+(1-\varepsilon) \boldsymbol{\nabla} \boldsymbol{u}_{h}^{n+1}: \boldsymbol{\nabla} \boldsymbol{v}+\frac{\varepsilon}{\mathrm{Wi}} \boldsymbol{\sigma}_{h}^{n+1}: \boldsymbol{\nabla} \boldsymbol{v} \\
-p_{h}^{n+1} \operatorname{div} \boldsymbol{v}+q \operatorname{div} \boldsymbol{u}_{h}^{n+1}+\left(\frac{\boldsymbol{\sigma}_{h}^{n+1}-\boldsymbol{\sigma}_{h}^{n} \circ X^{n}\left(t^{n}\right)}{\Delta t}\right): \boldsymbol{\phi}-\left(\left(\boldsymbol{\nabla} \boldsymbol{u}_{h}^{n+1}\right) \boldsymbol{\sigma}_{h}^{n+1}+\boldsymbol{\sigma}_{h}^{n+1}\left(\boldsymbol{\nabla} \boldsymbol{u}_{h}^{n+1}\right)^{T}\right): \boldsymbol{\phi} \\
+\frac{1}{\mathrm{Wi}}\left(\boldsymbol{\sigma}_{h}^{n+1}-\boldsymbol{I}\right): \boldsymbol{\phi}+\sum_{k=1}^{N_{K}} h_{K_{k}}^{2} \int_{K_{k}} \nabla p_{h} \cdot \nabla q, \quad(4
\end{array}
$$

with a flow $X^{n}$ again computed with the projected field $P_{h}^{\text {rot }}\left(\boldsymbol{u}_{h}^{n}\right)$ through (4.10). Again, we are led to choose the projection operator $P_{h}^{\text {rot }}$ because the weak incompressibility property (4.7) is not satisfied by $\boldsymbol{u}_{h}^{n}$. The stabilization procedure used in (4.16) has been studied in [8]. Proposition 4.1 holds for system (4.16), its proof being similar to the case of Taylor-Hood finite element (see Sect. 4.3.2), since the additional term $\sum_{k=1}^{N_{K}} h_{K_{k}}^{2} \int_{K_{k}} \nabla p_{h} \cdot \nabla q$ is non negative with the test function used in the proof. Again, all this also holds mutadis mutandis for discretization of the advection terms by a discontinuous Galerkin method, and for the log-formulation.

\section{Positivity, free energy estimate And the Long-time issue}

Notice that both Propositions 3.5 and 3.6 impose a limitation on the time step which depends on the time iteration: $0<\Delta t<c_{0}$, where $c_{0} \equiv c_{0}\left(\boldsymbol{u}_{h}^{n}, \boldsymbol{\sigma}_{h}^{n}\right)$ is function of a time-dependent data. Thus, these existence results are weak insofar as the long-time existence of the discrete solutions is not ensured, i.e. if $\sum_{n=0}^{\infty} c_{0}\left(\boldsymbol{u}_{h}^{n}, \boldsymbol{\sigma}_{h}^{n}\right)<\infty$.

Yet, for the discretizations introduced above, we have also shown that at each time step, the solutions of those discretizations satisfy free energy estimates. This will now allow us to prove the long-time existence of the discrete solutions.

Remark 5.1. In this section, we concentrate for simplicity on the discretization using Scott-Vogelius finite elements for velocity-pressure, and piecewise constant approximations for the stress. However, similar results can be proven for the other discretization methods introduced in Section 4.3 and Appendix D, since the solutions satisfy a free energy estimate. 
TABLE 2. Summary of some possible finite elements for $\left(\boldsymbol{u}_{h}, p_{h}, \boldsymbol{\sigma}_{h} / \boldsymbol{\psi}_{h}\right)$ when $\boldsymbol{\sigma}_{h} / \boldsymbol{\psi}_{h} \in$ $\left(\mathbb{P}_{0}\right)^{\frac{d(d+1)}{2}}$, with some possible projections for the velocity field (see Sect. 4.3).

\begin{tabular}{|c|c|}
\hline $\begin{array}{l}\text { - Advection discretized by } \\
\text { - }\left(\boldsymbol{u}_{h}, p_{h}\right) \text { in } \ldots\end{array}$ & $\begin{array}{l}\text { Characteristics or DG } \\
\Rightarrow \text { equations modified }\end{array}$ \\
\hline $\begin{array}{l}\text { Scott-Vogelius } \\
\left(\mathbb{P}_{2}\right)^{d} \times \mathbb{P}_{1, \text { disc }}\end{array}$ & (nothing) \\
\hline $\begin{array}{l}\text { Taylor-Hood } \\
\left(\mathbb{P}_{2}\right)^{d} \times \mathbb{P}_{1}\end{array}$ & $\begin{array}{l}+P_{h}^{\text {rot }} \\
+ \text { Temam term }\end{array}$ \\
\hline $\begin{array}{l}\text { Crouzeix-Raviart } \\
\left(\mathbb{P}_{1}^{C R}\right)^{d} \times \mathbb{P}_{0}\end{array}$ & $\begin{array}{l}+P_{h}^{B D M}, P_{h}^{R T_{0}} \text { or } P_{h}^{\text {rot }} \\
+P_{h}\left(\boldsymbol{u}_{h}^{n}\right) \text { for Navier term }\end{array}$ \\
\hline stabilized $\left(\mathbb{P}_{1}\right)^{d} \times \mathbb{P}_{1}$ & $\begin{array}{l}+P_{h}^{\text {rot }} \\
+ \text { Temam term }\end{array}$ \\
\hline stabilized $\left(\mathbb{P}_{1}\right)^{d} \times \mathbb{P}_{0}$ & $\begin{array}{l}+P_{h}^{\text {rot }} \\
+ \text { Temam term }\end{array}$ \\
\hline
\end{tabular}

Proposition 5.2. For any initial condition $\left(\boldsymbol{u}_{h}^{0}, \boldsymbol{\sigma}_{h}^{0}\right)$ with $\boldsymbol{\sigma}_{h}^{0}$ symmetric positive definite, there exists a constant $c_{1} \equiv c_{1}\left(\boldsymbol{u}_{h}^{0}, \boldsymbol{\sigma}_{h}^{0}\right)>0$ such that, for any time step $0 \leq \Delta t<c_{1}$, there exists, for all iterations $n \in \mathbb{N},\left(\boldsymbol{u}_{h}^{n+1}, \boldsymbol{\sigma}_{h}^{n+1}\right)$ which is the unique solution to the system (3.6) (resp. (3.8)) with $\boldsymbol{\sigma}_{h}^{n+1}$ symmetric positive definite.

Proof of Proposition 5.2. Like in the proof of Proposition 3.5, we will proceed with the proof for system (3.6) only, using its restatement as system (3.12).

The proof is by induction on the time index $n$. With the notation of the proof of Proposition 3.5, for a fixed $n=0, \ldots, N_{T}-1$ and for a fixed vector $Y^{n}$ of values in the subset $S_{+}^{*}$ of $\mathbb{R}^{2 N_{D}+3 N_{K}}$ (standing for the nodal and elementwise values of a field $\left(\boldsymbol{u}_{h}^{n}, \boldsymbol{\sigma}_{h}^{n}\right)$ with $\boldsymbol{\sigma}_{h}^{n}$ symmetric positive definite), we define like in the proof of Proposition 3.5 (using the implicit function theorem) a function $R: \Delta t \in\left[0, c_{0}\right) \rightarrow R(\Delta t) \in \mathbb{R}^{2 N_{D}+3 N_{K}}$ (where $\left.c_{0}=c_{0}\left(\boldsymbol{u}_{h}^{n}, \boldsymbol{\sigma}_{h}^{n}\right)\right)$ such that:

$$
\forall \Delta t \in\left[0, c_{0}\right), Q(\Delta t, R(\Delta t))=0
$$

where $Q$ is defined by (3.15). For any $\Delta t \in\left[0, c_{0}\right), R(\Delta t) \in \mathbb{R}^{2 N_{D}+3 N_{K}}$ stands for the nodal and elementwise values of a field $\left(\boldsymbol{u}_{h}(\Delta t), \boldsymbol{\sigma}_{h}(\Delta t)\right)$ (with $\boldsymbol{\sigma}_{h}(\Delta t)$ symmetric positive definite) that is solution to the system (3.12).

Then, by Proposition 4.1, the solution $\left(\boldsymbol{u}_{h}(\Delta t), \boldsymbol{\sigma}_{h}(\Delta t)\right)$ satisfies a free energy estimate:

$$
F\left(\boldsymbol{u}_{h}(\Delta t), \boldsymbol{\sigma}_{h}(\Delta t)\right) \leq F\left(\boldsymbol{u}_{h}^{n}, \boldsymbol{\sigma}_{h}^{n}\right)
$$

Using the fact that all norms are equivalent in the finite-dimensional vector space $\mathbb{R}^{2 N_{D}+3 N_{K}}$, and that, for $0<\nu \leq 1-\frac{1}{e}$, we have $\nu x \leq x-\ln (x), \forall x>0$, we obtain that there exists two constants $\alpha>0$ and $\beta>0$ (independent of $\Delta t$ ), such that:

$$
\alpha\|R(\Delta t)\| \leq F\left(\boldsymbol{u}_{h}(\Delta t), \boldsymbol{\sigma}_{h}(\Delta t)\right)+\beta
$$

Let us define the function $D$ :

$$
D: \Delta t \in\left[0, c_{0}\right) \longrightarrow B\left(Y^{n}\right)+A(R(\Delta t))+\left(\nabla_{Y} A\right) R(\Delta t) \in \mathbb{R}^{2 N_{D}+3 N_{K}} .
$$


We recall that (see (3.16)), with the new notations: $\nabla_{Y} Q(\Delta t, R(\Delta t))=I+\Delta t D(\Delta t)$. Using (5.1), (5.2) and the fact that the discrete free energy is non-increasing, the function $D$ satisfies:

$$
\begin{aligned}
\|D(\Delta t)\| & \leq\|B\|\left\|Y^{n}\right\|+\left(\|A\|+\left\|\nabla_{Y} A\right\|\right)\|R(\Delta t)\| \\
& \leq\left(\|B\|+\|A\|+\left\|\nabla_{Y} A\right\|\right) \frac{1}{\alpha}\left(F\left(\boldsymbol{u}_{h}^{n}, \boldsymbol{\sigma}_{h}^{n}\right)+\beta\right) \\
& \leq\left(\|B\|+\|A\|+\left\|\nabla_{Y} A\right\|\right) \frac{1}{\alpha}\left(F\left(\boldsymbol{u}_{h}^{0}, \boldsymbol{\sigma}_{h}^{0}\right)+\beta\right) .
\end{aligned}
$$

This shows that there exists a constant $c_{1} \equiv c_{1}\left(\boldsymbol{u}_{h}^{0}, \boldsymbol{\sigma}_{h}^{0}\right)>0$ such that, for any time step $0 \leq \Delta t<c_{1}$, the matrix $\nabla_{Y} Q(\Delta t, R(\Delta t))$ is invertible. Using the implicit function theorem, this implies that, for any time step $0 \leq \Delta t<c_{1}$, the system (3.12) admits a solution $\left(\boldsymbol{u}_{h}^{n+1}, \boldsymbol{\sigma}_{h}^{n+1}\right)$ with $\boldsymbol{\sigma}_{h}^{n+1}$ symmetric positive definite at all iterations $n \in \mathbb{N}$.

A similar result can be proven for the log-formulations (3.10) and (3.11):

Proposition 5.3. For any initial condition $\left(\boldsymbol{u}_{h}^{0}, \boldsymbol{\psi}_{h}^{0}\right)$, there exists a constant $c_{1} \equiv c_{1}\left(\boldsymbol{u}_{h}^{0}, \boldsymbol{\psi}_{h}^{0}\right)>0$ such that, for any time step $0 \leq \Delta t<c_{1}$, there exists, for all iterations $n \in \mathbb{N},\left(\boldsymbol{u}_{h}^{n+1}, \boldsymbol{\psi}_{h}^{n+1}\right)$ which is the unique solution to the system (3.10) (resp. (3.11)).

Proof of Proposition 5.3. The proof of Proposition 5.3 is similar to that of Proposition 5.2 using for $Q(\Delta t, Y)$ and $D(\Delta t)$ slightly modified expressions as explained for the proof of Proposition 3.6. The entropic term in the free energy still helps in bounding the norm of the vector of nodal-elementwise values for $\left(\boldsymbol{u}_{h}, \boldsymbol{\psi}_{h}\right)$ like in (5.2) using the following scalar inequality, which is true for any fixed $\nu \in(0,1]: \forall x \in \mathbb{R}, \mathrm{e}^{x}-x+1 \geq \nu|x|$.

From Propositions 5.2 and 5.3, we have the global-in-time existence of solutions to those discretizations of the Oldroyd-B system presented above which satisfy a discrete free energy estimate.

The log-formulation actually also satisfies the following long-time existence result, using the fact that the a priori estimates can be obtained without requiring the stress tensor field to be positive definite:

Proposition 5.4. For any initial condition $\left(\boldsymbol{u}_{h}^{0}, \boldsymbol{\psi}_{h}^{0}\right)$, and for any constant time step $\Delta t>0$, there exists, for all iterations $n \in \mathbb{N},\left(\boldsymbol{u}_{h}^{n+1}, \boldsymbol{\psi}_{h}^{n+1}\right)$ which is a solution to the system (3.10) (resp. (3.11)).

Proof of Proposition 5.4. We will proceed with the proof for system (3.10) only, using its restatement as system (3.14). Note already that, since the derivation of discrete free energy estimates for the system (3.10) does not require the solution $\psi_{h}^{n+1}$ and the test function to be non-negative like in the derivation of discrete free energy estimates for the system (3.6), then the manipulations used to derive the free energy estimate (4.5) can also be done a priori for any function in the finite element space.

Let us consider a fixed time index $n$ and a given couple $\left(\boldsymbol{u}_{h}^{n}, \boldsymbol{\psi}_{h}^{n}\right) \in\left(\mathbb{P}_{2}\right)_{\text {div }=0}^{d} \times\left(\mathbb{P}_{0}\right)^{\frac{d(d+1)}{2}}$. We equip the Hilbert space $\left(\mathbb{P}_{2}\right)_{\text {div }=0}^{d} \times\left(\mathbb{P}_{0}\right)^{\frac{d(d+1)}{2}}$ with the following inner product:

$$
\left(\left(\boldsymbol{v}_{1}, \phi_{1}\right) ;\left(\boldsymbol{v}_{2}, \phi_{2}\right)\right)=\int_{\mathcal{D}} \boldsymbol{v}_{1} \cdot \boldsymbol{v}_{2}+\phi_{1}: \phi_{2}
$$

for all $\left(\boldsymbol{v}_{1}, \phi_{1}\right),\left(\boldsymbol{v}_{2}, \boldsymbol{\phi}_{2}\right) \in\left(\mathbb{P}_{2}\right)_{\text {div }=0}^{d} \times\left(\mathbb{P}_{0}\right)^{\frac{d(d+1)}{2}}$, and denote by $\|\cdot\|$ the associated norm. Let us introduce the mapping $\mathcal{F}:\left(\mathbb{P}_{2}\right)_{\text {div }=0}^{d} \times\left(\mathbb{P}_{0}\right)^{\frac{d(d+1)}{2}} \rightarrow\left(\mathbb{P}_{2}\right)_{\text {div }=0}^{d} \times\left(\mathbb{P}_{0}\right)^{\frac{d(d+1)}{2}}$ defined by duality for all $(\boldsymbol{u}, \boldsymbol{\psi}) \in\left(\mathbb{P}_{2}\right)_{\text {div }=0}^{d} \times$ $\left(\mathbb{P}_{0}\right)^{\frac{d(d+1)}{2}}$ through the form:

$$
\begin{aligned}
(\mathcal{F}(\boldsymbol{u}, \boldsymbol{\psi}) ;(\boldsymbol{v}, \boldsymbol{\phi}))=\int_{\mathcal{D}} & \operatorname{Re}\left(\frac{\boldsymbol{u}-\boldsymbol{u}_{h}^{n}}{\Delta t}+\boldsymbol{u}_{h}^{n} \cdot \boldsymbol{\nabla} \boldsymbol{u}\right) \cdot \boldsymbol{v}+(1-\varepsilon) \boldsymbol{\nabla} \boldsymbol{u}: \boldsymbol{\nabla} \boldsymbol{v}+\frac{\varepsilon}{\mathrm{Wi}} \mathrm{e}^{\psi}: \boldsymbol{\nabla} \boldsymbol{v} \\
& +\left(\frac{\boldsymbol{\psi}-\boldsymbol{\psi}_{h}^{n} \circ X^{n}\left(t^{n}\right)}{\Delta t}\right): \boldsymbol{\phi}-(\boldsymbol{\Omega} \boldsymbol{\psi}-\boldsymbol{\psi} \boldsymbol{\Omega}): \boldsymbol{\phi}-2 \boldsymbol{B}: \boldsymbol{\phi}-\frac{1}{\mathrm{Wi}}\left(\mathrm{e}^{-\boldsymbol{\psi}}-\boldsymbol{I}\right): \boldsymbol{\phi}
\end{aligned}
$$


for any test function $(\boldsymbol{v}, \phi) \in\left(\mathbb{P}_{2}\right)_{\text {div }=0}^{d} \times\left(\mathbb{P}_{0}\right)^{\frac{d(d+1)}{2}}$, where we have used the decomposition of the velocity gradient $\boldsymbol{\nabla} \boldsymbol{u}$ as explained in Lemma 2.3:

$$
\nabla \boldsymbol{u}=\boldsymbol{\Omega}+\boldsymbol{B}+\boldsymbol{N} \mathrm{e}^{-\psi},
$$

with $\boldsymbol{\Omega}$ and $\boldsymbol{B}$ continuous with respect to $\boldsymbol{\nabla} \boldsymbol{u}$, so that $\mathcal{F}$ is a continuous mapping on finite balls of radius $\alpha>0$ :

$$
\mathcal{B}_{\alpha}=\left\{(\boldsymbol{v}, \phi) \in\left(\mathbb{P}_{2}\right)_{\operatorname{div}=0}^{d} \times\left(\mathbb{P}_{0}\right)^{\frac{d(d+1)}{2}},\|(\boldsymbol{v}, \phi)\| \leq \alpha\right\}
$$

Note that if $\left(\boldsymbol{u}_{h}^{n+1}, \boldsymbol{\psi}_{h}^{n+1}\right)$ is a solution to (3.14), then we have: for all $(\boldsymbol{v}, \boldsymbol{\phi}) \in\left(\mathbb{P}_{2}\right)_{\operatorname{div}=0}^{d} \times\left(\mathbb{P}_{0}\right)^{\frac{d(d+1)}{2}}$,

$$
\left(\mathcal{F}\left(\boldsymbol{u}_{h}^{n+1}, \boldsymbol{\psi}_{h}^{n+1}\right) ;(\boldsymbol{v}, \phi)\right)=0 .
$$

Let us now assume that the mapping $\mathcal{F}$ has no zero $\left(\boldsymbol{u}_{h}^{n+1}, \boldsymbol{\psi}_{h}^{n+1}\right)$ satisfying (5.3) in the ball $\mathcal{B}_{\alpha}$. Then, we define the following continuous mapping from $\mathcal{B}_{\alpha}$ onto itself $(\mathcal{F}$ is continuous on the finite-dimensional compact, convex ball $\mathcal{B}_{\alpha}$ ):

$$
\mathcal{G}(\boldsymbol{v}, \phi)=-\alpha \frac{\mathcal{F}(\boldsymbol{v}, \phi)}{\|\mathcal{F}(\boldsymbol{v}, \phi)\|}, \forall(\boldsymbol{v}, \phi) \in\left(\mathbb{P}_{2}\right)_{\text {div }=0}^{d} \times\left(\mathbb{P}_{0}\right)^{\frac{d(d+1)}{2}} .
$$

By the Brouwer fixed point theorem, $\mathcal{G}$ has a fixed point in $\mathcal{B}_{\alpha}$. Let us still denote that fixed point $(\boldsymbol{v}, \boldsymbol{\phi})$ for the sake of simplicity. By definition, it satisfies:

$$
\mathcal{G}(\boldsymbol{v}, \phi)=(\boldsymbol{v}, \phi) \in \mathcal{B}_{\alpha} \text { and }\|\mathcal{G}(\boldsymbol{v}, \phi)\|=\alpha .
$$

Considering $\mathcal{F}(\boldsymbol{v}, \phi)$ and using $\left(\boldsymbol{v}, \frac{\varepsilon}{2 W \mathrm{i}}\left(\mathrm{e}^{\phi}-\boldsymbol{I}\right)\right)$ as a test function, we get the following inequality after similar manipulations to those in the proof of Proposition 4.3:

$$
\begin{aligned}
\left(\mathcal{F}(\boldsymbol{v}, \phi) ;\left(\boldsymbol{v}, \frac{\varepsilon}{2 \mathrm{Wi}}\left(\mathrm{e}^{\phi}-\boldsymbol{I}\right)\right)\right) \geq & \frac{\operatorname{Re}}{2} \int_{\mathcal{D}}|\boldsymbol{v}|^{2}+\frac{\varepsilon}{2 \mathrm{Wi}} \int_{\mathcal{D}} \operatorname{tr}\left(\mathrm{e}^{\phi}-\phi\right)-\frac{\operatorname{Re}}{2} \int_{\mathcal{D}}\left|\boldsymbol{u}_{h}^{n}\right|^{2}-\frac{\varepsilon}{2 \mathrm{Wi}} \int_{\mathcal{D}} \operatorname{tr}\left(\mathrm{e}^{\boldsymbol{\psi}_{h}^{n}}-\boldsymbol{\psi}_{h}^{n}\right) \\
& +\int_{\mathcal{D}} \frac{\operatorname{Re}}{2}\left|\boldsymbol{v}-\boldsymbol{u}_{h}^{n}\right|^{2}+\Delta t \int_{\mathcal{D}}(1-\varepsilon)\left|\boldsymbol{\nabla} \boldsymbol{u}_{h}^{n+1}\right|^{2}+\frac{\varepsilon}{2 \mathrm{Wi}^{2}} \operatorname{tr}\left(\mathrm{e}^{\phi}+\mathrm{e}^{-\phi}-2 I\right) .
\end{aligned}
$$

Then, using the scalar inequality $\mathrm{e}^{x}-x \geq|x|, \forall x \in \mathbb{R}$, we have:

$$
\int_{\mathcal{D}} \operatorname{tr}\left(\mathrm{e}^{\phi}-\boldsymbol{\phi}+\boldsymbol{I}\right) \geq \sum_{i=1}^{d} \int_{\mathcal{D}}\left|\lambda_{i}\right|, \forall \boldsymbol{\phi} \in\left(\mathbb{P}_{0}\right)^{\frac{d(d+1)}{2}},
$$

where $\left(\lambda_{i}\right)_{1 \leq i \leq d}$ are functions depending on $\boldsymbol{\phi}$ such that, for all $\boldsymbol{x} \in \mathcal{D},\left(\lambda_{i}(\boldsymbol{x})\right)_{1 \leq i \leq d}$ are the $d$ (non-necessarily distinct) real eigenvalues of the symmetric matrix $\phi(x)$. Now, since $\left(\mathbb{P}_{2}\right)_{\text {div }=0}^{d} \times\left(\mathbb{P}_{0}\right)^{\frac{d(d+1)}{2}}$ is finite-dimensional, all norms are equivalent. So there exist $\gamma_{1}, \gamma_{2}>0$ such that, for all $(\boldsymbol{v}, \phi) \in\left(\mathbb{P}_{2}\right)_{\operatorname{div}=0}^{d} \times\left(\mathbb{P}_{0}\right)^{\frac{d(d+1)}{2}}$ :

$$
\gamma_{1}\|(\boldsymbol{v}, \boldsymbol{\phi})\| \leq\left(\int_{\mathcal{D}}|\boldsymbol{v}|^{2}\right)^{\frac{1}{2}}+\left\|\max _{1 \leq i \leq d}\left|\lambda_{i}(\boldsymbol{x})\right|\right\|_{\infty} \leq \gamma_{2}\|(\boldsymbol{v}, \boldsymbol{\phi})\|
$$


where it is easy to prove that $\left\|\max _{1 \leq i \leq d}\left|\lambda_{i}(\boldsymbol{x})\right|\right\|_{\infty}$ defines a norm in the vector space $L^{\infty}\left(\mathcal{D}, \mathcal{S}\left(\mathbb{R}^{d \times d}\right)\right)$. Using the equation (5.6) with the norm equivalence (5.7), we obtain:

$$
\begin{aligned}
\frac{\operatorname{Re}}{2} \int_{\mathcal{D}}|\boldsymbol{v}|^{2}+ & \frac{\varepsilon}{2 \mathrm{Wi}} \int_{\mathcal{D}} \operatorname{tr}\left(\mathrm{e}^{\phi}-\boldsymbol{\phi}+\boldsymbol{I}\right) \\
& \geq \min \left(\frac{\operatorname{Re}}{2}, \frac{\varepsilon}{2 \mathrm{Wi}} \frac{1}{\left\|\max _{1 \leq i \leq d}\left|\lambda_{i}(\boldsymbol{x})\right|\right\|_{\infty}}\right)\left(\int_{\mathcal{D}}|\boldsymbol{v}|^{2}+\left\|\max _{1 \leq i \leq d}\left|\lambda_{i}(\boldsymbol{x})\right|\right\|_{\infty} \sum_{i=1}^{d} \int_{\mathcal{D}}\left|\lambda_{i}\right|\right) \\
& \geq \min \left(\frac{\operatorname{Re}}{2}, \frac{\varepsilon}{2 \mathrm{Wi}} \frac{1}{\left\|\max _{1 \leq i \leq d}\left|\lambda_{i}(\boldsymbol{x})\right|\right\|_{\infty}}\right)\left(\int_{\mathcal{D}}|\boldsymbol{v}|^{2}+\sum_{i=1}^{d} \int_{\mathcal{D}}\left|\lambda_{i}\right|^{2}\right) .
\end{aligned}
$$

Last, since the fixed-point $(\boldsymbol{v}, \phi) \in \mathcal{B}_{\alpha}$ satisfies $\|(\boldsymbol{v}, \phi)\|=\alpha$ because of (5.4), we can choose $\alpha$ large enough so that:

and we get:

$$
\min \left(\frac{\operatorname{Re}}{2}, \frac{\varepsilon}{2 \mathrm{Wi} \gamma_{2} \alpha}\right)\|(\boldsymbol{v}, \phi)\|^{2}>\frac{\operatorname{Re}}{2} \int_{\mathcal{D}}\left|\boldsymbol{u}_{h}^{n}\right|^{2}+\frac{\varepsilon}{2 \mathrm{Wi}} \int_{\mathcal{D}} \operatorname{tr}\left(\mathrm{e}^{\psi_{h}^{n}}-\boldsymbol{\psi}_{h}^{n}+\boldsymbol{I}\right),
$$

$$
\begin{aligned}
\frac{\operatorname{Re}}{2} \int_{\mathcal{D}}|\boldsymbol{v}|^{2}+\frac{\varepsilon}{2 \mathrm{Wi}} \int_{\mathcal{D}} \operatorname{tr}\left(\mathrm{e}^{\phi}-\boldsymbol{\phi}+\boldsymbol{I}\right) & -\frac{\operatorname{Re}}{2} \int_{\mathcal{D}}\left|\boldsymbol{u}_{h}^{n}\right|^{2}-\frac{\varepsilon}{2 \mathrm{Wi}} \int_{\mathcal{D}} \operatorname{tr}\left(\mathrm{e}^{\boldsymbol{\psi}_{h}^{n}}-\boldsymbol{\psi}_{h}^{n}+\boldsymbol{I}\right) \\
& +\int_{\mathcal{D}} \frac{\operatorname{Re}}{2}\left|\boldsymbol{v}-\boldsymbol{u}_{h}^{n}\right|^{2}+\Delta t \int_{\mathcal{D}}(1-\varepsilon)\left|\boldsymbol{\nabla} \boldsymbol{u}_{h}^{n+1}\right|^{2}+\frac{\varepsilon}{2 \mathrm{Wi}^{2}} \operatorname{tr}\left(\mathrm{e}^{\phi}+\mathrm{e}^{-\boldsymbol{\phi}}-2 \boldsymbol{I}\right)>0,
\end{aligned}
$$

that is:

Now, using the equation (5.4) we have:

$$
\left(\mathcal{F}(\boldsymbol{v}, \boldsymbol{\phi}) ;\left(\boldsymbol{v}, \frac{\varepsilon}{2 \mathrm{Wi}}\left(\mathrm{e}^{\phi}-\boldsymbol{I}\right)\right)\right)>0 .
$$

$$
\left(\mathcal{F}(\boldsymbol{v}, \phi) ;\left(\boldsymbol{v}, \frac{\varepsilon}{2 \mathrm{Wi}}\left(\mathrm{e}^{\phi}-\boldsymbol{I}\right)\right)\right)=-\frac{\|\mathcal{F}(\boldsymbol{v}, \boldsymbol{\phi})\|}{\alpha}\left(\int_{\mathcal{D}}|\boldsymbol{v}|^{2}+\frac{\varepsilon}{2 \mathrm{Wi}} \operatorname{tr}\left(\phi \mathrm{e}^{\phi}-\phi\right)\right) \leq 0
$$

which is obviously in contradiction with (5.8) since, for all $\phi \in\left(\mathbb{P}_{0}\right)^{\frac{d(d+1)}{2}}$, we have $\operatorname{tr}\left(\phi \mathrm{e}^{\phi}-\phi\right) \geq 0$ by virtue of the scalar inequality $x\left(\mathrm{e}^{x}-1\right) \geq 0, \forall x \in \mathbb{R}$.

Thus, for any $\Delta t>0$, if we choose $\alpha$ sufficiently large, the mapping $\mathcal{F}$ has a zero $\left(\boldsymbol{u}_{h}^{n+1}, \boldsymbol{\psi}_{h}^{n+1}\right)$ satisfying (5.3) in the ball $\mathcal{B}_{\alpha}$, which concludes the proof.

Notice that Proposition 5.4 does not ensure the uniqueness of solutions. There may be bifurcations, hence many possible solutions to the log-formulation, in the case where the CFL condition is not fulfilled. Though, all those solutions will satisfy a free energy estimate, which is not the case for the usual formulation in terms of $\boldsymbol{\tau}$. The fact that we are able to prove such a stability result without any assumption on the timestep for the log-formulation, and not for the classical formulation, may be related to the fact that discretizations of the log-formulation have been reported to yield solutions beyond the limiting Weissenberg number for standard discretizations (see [23]).

Remark 5.5 (other positivity preserving schemes). There exist other means than using the log-formulation to preserve the non-negativity of the conformation tensor. A very natural way of preserving the non-negativity is to reformulate the constitutive equation with the deformation gradient instead of the stress or the conformation tensor, using a Lie-derivative like in [32]. It is also possible to build free-energy-dissipative schemes for a Lieformulation, as shown in Appendix E. But discretizations of a Lie-formulation seem to necessitate the numerical integration of ordinary differential equations like (3.7) for the characteristic flow, which may introduce new instabilities (see Rem. 3.2). 


\section{Appendix A. Some Properties of Symmetric Positive Definite MATRices}

\section{A.1. Proof of Lemma 1.1}

Formula (1.6), (1.7) and (1.8) are simply obtained by diagonalizing the symmetric positive definite matrix $\boldsymbol{\sigma}$, and using the inequalities: $\forall x, y>0, \ln (x y)=\ln x+\ln y, x-1 \geq \ln x$ and $x+1 / x \geq 2$.

Let us now prove formula (1.9). By diagonalization, we have $\bar{\sigma}=\Omega^{T} D \Omega$ with $\Omega$ orthogonal and $D$ diagonal positive, which gives:

$$
\operatorname{tr}\left(\boldsymbol{\sigma} \tau^{-1}\right)=\operatorname{tr}\left(\Omega^{T} \sqrt{D} \sqrt{D} \Omega \boldsymbol{\tau}^{-1}\right)=\operatorname{tr}\left(\sqrt{D} \Omega \boldsymbol{\tau}^{-1} \Omega^{T} \sqrt{D}\right) \geq 0,
$$

because $A=\sqrt{D} \Omega \tau^{-1} \Omega^{T} \sqrt{D}$ is clearly a symmetric positive definite matrix. Likewise, we have:

$$
\operatorname{det}\left(\boldsymbol{\sigma} \tau^{-1}\right)=\operatorname{det}\left(\Omega^{T} D \Omega \tau^{-1}\right)=\operatorname{det}\left(\sqrt{D} \Omega \tau^{-1} \Omega^{T} \sqrt{D}\right) .
$$

The proof of (1.10) is then equivalent to show:

$$
\ln (\operatorname{det}(A)) \leq \operatorname{tr}(A-\boldsymbol{I}),
$$

for any symmetric positive definite matrix $A$, which simply derives from (1.6) and (1.7).

It remains to prove formula (1.11). By diagonalization, we write $\boldsymbol{\sigma}=O^{T} D O$ and $\boldsymbol{\tau}=R^{T} \Lambda R$ with $O$ and $R$ orthogonal, and $D$ and $\Lambda$ diagonal positive. Let us introduce the orthogonal matrix $\Omega=O R^{T}$. We denote by $D_{i}\left(\right.$ resp. $\left.\Lambda_{i}\right)$ the $(i, i)$-th entry of $D($ resp. of $\Lambda)$. We have:

$$
\begin{aligned}
\operatorname{tr}((\ln \boldsymbol{\sigma}-\ln \boldsymbol{\tau}) \boldsymbol{\sigma}-(\boldsymbol{\sigma}-\boldsymbol{\tau})) & =\sum_{i} D_{i} \ln D_{i}-D_{i}+\Lambda_{i}-\sum_{i, j}\left(\Omega_{i j}\right)^{2} D_{i} \ln \Lambda_{j} \\
& =\sum_{i}\left(\Lambda_{i}-D_{i}-\sum_{j}\left(\Omega_{i j}\right)^{2} D_{i}\left(\ln \Lambda_{j}-\ln D_{i}\right)\right)
\end{aligned}
$$

since $\Omega$ is an orthogonal matrix $\left(\sum_{j}\left(\Omega_{i j}\right)^{2}=1\right.$ for all $\left.i\right)$. Using the convexity inequality $x-y \leq x(\ln x-\ln y)$ for all $x, y>0$, we thus obtain $\operatorname{tr}((\ln \boldsymbol{\sigma}-\ln \boldsymbol{\tau}) \boldsymbol{\sigma}-(\boldsymbol{\sigma}-\boldsymbol{\tau})) \geq 0$ which concludes the proof of (1.11).

\section{A.2. Proof of Lemma 1.2}

First, since $\boldsymbol{\sigma} \in\left(C^{1}([0, T))\right)^{\frac{d(d+1)}{2}}$ is symmetric positive definite, $\operatorname{det}(\boldsymbol{\sigma})$ is positive and $C^{1}([0, T))$. So we immediately get the classical Jacobi formula (1.12):

$$
\frac{\mathrm{d}}{\mathrm{d} t} \ln (\operatorname{det}(\boldsymbol{\sigma}))=(1 / \operatorname{det}(\boldsymbol{\sigma})) \frac{\mathrm{d}}{\mathrm{d} t} \operatorname{det}(\boldsymbol{\sigma})=\operatorname{tr}\left(\boldsymbol{\sigma}^{-1} \frac{\mathrm{d}}{\mathrm{d} t} \boldsymbol{\sigma}\right),
$$

on noting that $\ln (\operatorname{det}(\boldsymbol{\sigma}))=\operatorname{tr}(\ln (\boldsymbol{\sigma}))$.

Then, for the proof of (1.13), first note that the matrix exponential is a $C^{\infty}$-diffeomorphism from the set of symmetric matrices onto the set of symmetric positive definite matrices by virtue of the local inversion theorem (see [37], Cor. 3.8.5, for instance), whose inverse mapping coincides with the matrix logarithm defined in (1.5). Then, there exists $\boldsymbol{\tau} \in\left(C^{1}([0, T))\right)^{\frac{d(d+1)}{2}}$ such that $\boldsymbol{\sigma}=\mathrm{e}^{\boldsymbol{\tau}}$, and on noting that $\boldsymbol{\sigma}$ and $\boldsymbol{\tau}$ commute, we immediately get (1.13):

$$
\operatorname{tr}\left(\sigma \frac{\mathrm{d} \ln \sigma}{\mathrm{d} t}\right)=\operatorname{tr}\left(\mathrm{e}^{\tau} \frac{\mathrm{d} \boldsymbol{\tau}}{\mathrm{d} t}\right)=\operatorname{tr}\left(\frac{\mathrm{de}}{\mathrm{d} t}\right)=\frac{\mathrm{d}}{\mathrm{d} t} \operatorname{tr} \boldsymbol{\sigma} .
$$




\section{Appendix B. Proof of Lemma 2.1}

Let us introduce $t_{0}=\inf \{t>0 \mid \boldsymbol{\sigma}(t)$ is not symmetric positive definite $\}$, with convention $t_{0}=\infty$ if $\{t>0, \boldsymbol{\sigma}$ is not symmetric positive definite $\}=\emptyset$. Since $\boldsymbol{\sigma}(t=0)$ is symmetric positive definite, it remains so at least for small times $0 \leq t<\Delta t$, by continuity of $\operatorname{det}(\boldsymbol{\sigma})$ with respect to the time variable $t$. Thus, $t_{0} \geq \Delta t>0$.

Let us assume that $t_{0}<\infty$. First, one can define the logarithm $\ln \boldsymbol{\sigma}$ of $\boldsymbol{\sigma}$, which satisfies the equation for $\boldsymbol{\psi}$ in system (2.10) for $t \in\left[0, t_{0}\right)$. Taking the trace of the equation for $\boldsymbol{\psi}$ in system (2.10), we get for $\ln \boldsymbol{\sigma}$ :

$$
\frac{\mathrm{D}}{\mathrm{D} t} \ln \operatorname{det} \boldsymbol{\sigma}=\frac{1}{\mathrm{Wi}} \operatorname{tr}\left(\boldsymbol{\sigma}^{-1}-\boldsymbol{I}\right)
$$

where we have introduced the convective derivative $\frac{\mathrm{D}}{\mathrm{D} t}=\left(\frac{\mathrm{d}}{\mathrm{d} t}+(\boldsymbol{u} \cdot \boldsymbol{\nabla})\right)$ (the next formulae (B.3) and (B.4) thus hold along the characteristics, which are well defined because $\left.\boldsymbol{u} \in C^{1}\left([0, T), C^{0,1}(\mathcal{D})\right)\right)$. Besides, for any positive definite matrix $\boldsymbol{\sigma}^{-1}$, we have:

$$
\frac{\operatorname{tr}\left(\boldsymbol{\sigma}^{-1}\right)}{d} \geq\left(\operatorname{det} \boldsymbol{\sigma}^{-1}\right)^{1 / d}
$$

which follows from the convex inequality between geometrical and arithmetical means. Thus, combining (B.1) and (B.2), we get, on the time interval $\left[0, t_{0}\right)$ :

$$
\frac{\mathrm{D}}{\mathrm{D} t}(\operatorname{det} \boldsymbol{\sigma})^{1 / d}=\frac{1}{d}(\operatorname{det} \boldsymbol{\sigma})^{1 / d} \frac{\mathrm{D}}{\mathrm{D} t} \ln \operatorname{det} \boldsymbol{\sigma} \geq \frac{1}{\mathrm{Wi}}\left(1-(\operatorname{det} \boldsymbol{\sigma})^{1 / d}\right) .
$$

Now, by continuity of $\operatorname{det}(\boldsymbol{\sigma})$ with respect to $t$, one eigenvalue at least converges to zero as $t \rightarrow t_{0}^{-}$, which implies det $\boldsymbol{\sigma} \rightarrow 0^{+}$. Then, there exists $\eta>0$ such that, for times $t_{0}-\eta<t<t_{0}$, we have:

$$
0<\operatorname{det} \sigma<1,
$$

and by (B.3):

$$
\frac{\mathrm{D}}{\mathrm{D} t}(\operatorname{det} \boldsymbol{\sigma})^{1 / d}>0
$$

But then, $t_{0}$ cannot be the first time when $\operatorname{det} \boldsymbol{\sigma}=0$, otherwise one should have $\frac{\mathrm{D}}{\mathrm{D} t}(\operatorname{det} \boldsymbol{\sigma})^{1 / d}\left(t_{0}^{-}\right) \leq 0$, which contradicts (B.4). Thus $t_{0}=\infty$ which ends the proof of Lemma 2.1.

\section{Appendix C. Proof of Lemmas 2.3 And 3.3}

Lemmas 2.3 and 3.3 are consequences of the following result, which is a slight modification of a result proved in $[14]$.

Lemma C.1. Let $\boldsymbol{M}$ be a $d \times d$ matrix and $\boldsymbol{\sigma}$ be a symmetric positive definite $d \times d$ matrix. Then, there exists three $d \times d$ matrices $\boldsymbol{\Omega}, \boldsymbol{B}$ and $\boldsymbol{N}$ such that

$$
M=\Omega+B+N \sigma^{-1}
$$

and $\boldsymbol{B}$ is a symmetric matrix which commutes with $\boldsymbol{\sigma}, \boldsymbol{\Omega}$ and $\boldsymbol{N}$ are antisymmetric. Moreover, the entries of $\boldsymbol{\Omega}, \boldsymbol{B}$ and $\boldsymbol{N}$ are linear with respect to the entries of $\boldsymbol{M}$.

Proof. First, it is easy to check by diagonalization that it is sufficient to prove the result for a diagonal matrix $\boldsymbol{\sigma}$ (more precisely, by rewriting everything in a diagonalizing basis for $\boldsymbol{\sigma}$ ). In the following, we thus assume that $\boldsymbol{\sigma}=\operatorname{diag}\left(\Lambda_{1}, \ldots, \Lambda_{d}\right)$, where $\left(\Lambda_{i}\right)_{1 \leq i \leq d}$ are positive numbers. Moreover, we restrict ourselves to the physical case $d=3$, but the arguments can be generalized to any dimension. 
Let us first consider the case $\Lambda_{i} \neq \Lambda_{j}$ for $i \neq j$. In this case, we set:

- $B_{i, i}=M_{i, i}$ and $B_{i, j}=0$ for $i \neq j$;

- $N_{i, i}=0$ and $N_{i, j}=\frac{M_{i, j}+M_{j, i}}{\Lambda_{j}^{-1}-\Lambda_{i}^{-1}}$ for $i \neq j$;

- $\Omega_{i, i}=0$ and $\Omega_{i, j}=-\frac{M_{i, j} \Lambda_{i}^{-1}+M_{j, i} \Lambda_{j}^{-1}}{\Lambda_{j}^{-1}-\Lambda_{i}^{-1}}$ for $i \neq j$.

It is easy to check that these matrices satisfy the requirements of the lemma.

Let us now consider the case $\Lambda_{1}=\Lambda_{2}=\Lambda_{3}$. In this case, we simply set $\boldsymbol{N}=0, \boldsymbol{B}=\frac{\boldsymbol{M}+\boldsymbol{M}^{T}}{2}$ and $\boldsymbol{\Omega}=\frac{\boldsymbol{M}-\boldsymbol{M}^{T}}{2}$. It is again straightforward to check that these matrices satisfy the requirements of the lemma.

Finally, let us consider the case when only two $\Lambda_{i}$ 's are equal. Without loss of generality, we can suppose $\Lambda_{1}=\Lambda_{2} \neq \Lambda_{3}$. In this case, we set:

- $B_{3,3}=M_{3,3}, B_{i, j}=\frac{M_{i, j}+M_{j, i}}{2}$ for $1 \leq i, j \leq 2$ and $B_{i, j}=0$ otherwise;

- $N_{i, j}=0$ for $1 \leq i, j \leq 2, N_{3,3}=0$ and $N_{i, j}=\frac{M_{i, j}+M_{j, i}}{\Lambda_{j}^{-1}-\Lambda_{i}^{-1}}$ otherwise;

- $\Omega_{i, j}=\frac{M_{i, j}-M_{j, i}}{2}$ for $1 \leq i, j \leq 2, \Omega_{3,3}=0$ and $\Omega_{i, j}=-\frac{M_{i, j} \Lambda_{i}^{-1}+M_{j, i} \Lambda_{j}^{-1}}{\Lambda_{j}^{-1}-\Lambda_{i}^{-1}}$ otherwise.

This case is a combination of the two previous cases, and one can check that these matrices satisfy the requirements of the lemma.

Notice in particular that the linear dependence of the entries of the matrices $\boldsymbol{\Omega}, \boldsymbol{B}$ and $\boldsymbol{N}$ with respect to the entries of $\boldsymbol{M}$ implies that if $\boldsymbol{\sigma}$ is piecewise constant (with respect to the space variable) and $\boldsymbol{M}$ is $\left(\mathbb{P}_{k, \text { disc }}\right)^{d \times d}$, then $\boldsymbol{\Omega}, \boldsymbol{B}$ and $\boldsymbol{N}$ are also $\left(\mathbb{P}_{k, \text { disc }}\right)^{d \times d}$ (which is the result of Lem. 3.3).

\section{Appendix D. Higher order Discretization of the STREss Fields $\boldsymbol{\sigma}_{h}$ AND $\boldsymbol{\psi}_{h}$}

We now show how to build numerical schemes with higher order discretization spaces for the stress that still satisfy a discrete free energy estimate. We typically have in mind piecewise linear spaces for $\boldsymbol{\sigma}_{h}$ and $\boldsymbol{\psi}_{h}$.

From the previous proofs establishing discrete free energy estimates at low order in $\mathbb{P}_{0}$, it is clear that we need to use nonlinear functionals of $\boldsymbol{\sigma}_{h}$ and $\boldsymbol{\psi}_{h}$ as test functions, namely $\boldsymbol{\sigma}_{h}^{-1}$ and $\mathrm{e}^{\boldsymbol{\psi}_{h}}$. Finite element spaces other than $\mathbb{P}_{0}$ are typically not invariant under such nonlinear functionals, and this brings us to introduce projections of these nonlinear terms on $\mathbb{P}_{0}$, and finite element spaces to discretize the stress that contain $\mathbb{P}_{0}$, thus discontinuous.

We will use a $\mathbb{P}_{0}$-Lagrange interpolation operator $\pi_{h}$ which is convenient because it commutes with nonlinear functionals (see Lem. D.4 below). Moreover, we will need that this interpolation operator coincides with an $L^{2}$ orthogonal projection onto $\mathbb{P}_{0}$ (see Lem. D.3 below). The need for $\pi_{h}$ to coincide with an $L^{2}$ orthogonal projection onto $\mathbb{P}_{0}$ limits the maximum regularity of the discretization of the stress, essentially to piecewise $\mathbb{P}_{1}$ finite elements. Therefore, we consider $\boldsymbol{\sigma}_{h}$ and $\boldsymbol{\psi}_{h}$ in either of the following finite element spaces $^{1}$ :

$$
\left(\mathbb{P}_{1}+\mathbb{P}_{0}\right)^{\frac{d(d+1)}{2}} \text { or }\left(\mathbb{P}_{1, \text { disc }}\right)^{\frac{d(d+1)}{2}} .
$$

In Section D.1, we introduce the interpolation operator $\pi_{h}$. Then we prove that, for a Scott-Vogelius discretization of the velocity-pressure field, a free energy estimate can be obtained for discretization schemes close to those considered in Section 4 , when $\boldsymbol{\sigma}_{h}$ (respectively $\boldsymbol{\psi}_{h}$ ) is in $\left(\mathbb{P}_{0}\right)^{\frac{d(d+1)}{2}}$. This is the purpose of the Section D.2 (respectively Sect. D.3). Finally, we show in Section D.4 how these results can be extended to other finite element discretizations of the velocity-pressure field.

Remark D.1. In this appendix, we concentrate on establishing free energy estimates, and do not prove existence results as those stated in Sections 3.5 and 5. It is easy to extend these existence results to the numerical schemes considered here.

\footnotetext{
${ }^{1}$ Note that, clearly, $\left(\mathbb{P}_{1}+\mathbb{P}_{0}\right)^{\frac{d(d+1)}{2}}$ is only a subspace of $\left(\mathbb{P}_{1, \text { disc }}\right)^{\frac{d(d+1)}{2}}$.
} 


\section{D.1. The interpolation operator $\pi_{h}$}

Let us introduce the projection operator $\pi_{h}$ as the $\mathbb{P}_{0}$ Lagrange interpolation at barycenter $\theta_{K_{k}}$ for each $K_{k} \in \mathcal{T}_{h}$.

Definition D.2. For $k=1, \ldots, N_{K}$, we denote by $\theta_{K_{k}}$ the barycenter of the triangle $K_{k}$. For any $\phi$ such that $\forall k=1, \ldots, N_{K}, \phi\left(\theta_{K_{k}}\right)$ is well-defined (for example $\phi$ is a tensor-valued function, continuous at points $\theta_{K_{k}}$ ), we define its piecewise constant interpolation by:

$$
\forall k=1, \ldots, N_{K},\left.\pi_{h}(\phi)\right|_{K_{k}}=\phi\left(\theta_{K_{k}}\right) .
$$

Notice that this definition also makes sense for the case in which $\phi$ is matrix-valued. And this interpolation operator $\pi_{h}$ coincides with the $L^{2}$ orthogonal projection from $\left(\mathbb{P}_{1, \text { disc }}\right)^{\frac{d(d+1)}{2}}$ onto $\left(\mathbb{P}_{0}\right)^{\frac{d(d+1)}{2}}$ :

Lemma D.3. Let $\pi_{h}$ be the interpolation operator introduced in Definition D.2. Then, for any $\phi_{h} \in\left(\mathbb{P}_{1, \mathrm{disc}}\right)^{\frac{d(d+1)}{2}}$, we have:

$$
\int_{\mathcal{D}} \phi_{h}: \tilde{\phi}_{h}=\int_{\mathcal{D}} \pi_{h}\left(\phi_{h}\right): \tilde{\phi}_{h}, \forall \tilde{\phi}_{h} \in\left(\mathbb{P}_{0}\right)^{\frac{d(d+1)}{2}} .
$$

Proof. It is enough to prove Lemma D.3 on each simplex $K_{k} \in \mathcal{T}_{h}$ and in the scalar case. Let $\left(x_{i}\right)_{1 \leq i \leq 3}$ be the vertices of the simplex $K_{k}$ and $\left(\psi_{i}\right)_{1 \leq i \leq 3}$ the corresponding (linear) basis functions in $\mathbb{P}_{1}$. Then, the function $\left.\phi_{h}\right|_{K_{k}} \in \mathbb{P}_{1}$ reads $\left.\phi_{h}\right|_{K_{k}}(x)=\phi_{h}\left(x_{1}\right) \psi_{1}(x)+\phi_{h}\left(x_{2}\right) \psi_{2}(x)+\phi_{h}\left(x_{3}\right) \psi_{3}(x), \forall x \in K_{k}$. For every $\tilde{\phi}_{h} \in \mathbb{P}_{0}$,

$$
\int_{K_{k}} \phi_{h} \tilde{\phi}_{h}=\tilde{\phi_{h}}\left(\int_{K_{k}} \phi_{h}\right)=\tilde{\phi}_{h} \frac{\left|K_{k}\right|}{3}\left(\phi_{h}\left(x_{1}\right)+\phi_{h}\left(x_{2}\right)+\phi_{h}\left(x_{3}\right)\right)
$$

because $\int_{K_{k}} \psi_{i}=\frac{\left|K_{k}\right|}{3}$. Moreover, $\left.\phi_{h}\right|_{K_{k}} \in \mathbb{P}_{1}$, hence

$$
\frac{1}{3}\left(\phi_{h}\left(x_{1}\right)+\phi_{h}\left(x_{2}\right)+\phi_{h}\left(x_{3}\right)\right)=\phi_{h}\left(\frac{x_{1}+x_{2}+x_{3}}{3}\right)=\phi_{h}\left(\theta_{K_{k}}\right)
$$

which means

$$
\int_{K_{k}} \phi_{h} \tilde{\phi_{h}}=\int_{K_{k}} \tilde{\phi}_{h} \phi_{h}\left(\theta_{K_{k}}\right)=\int_{K_{k}} \pi_{h}(\phi) \tilde{\phi}_{h}
$$

In addition, the following property holds, which is important in the choice of this particular interpolation:

Lemma D.4. Let $\pi_{h}$ be the interpolation operator introduced in Definition D.2. The interpolation operator $\pi_{h}$ commutes with any function $f$ : for any functions $f$ and $\phi_{h}$ such that $\phi_{h}$ and $f\left(\phi_{h}\right)$ are well-defined at the barycenters $\theta_{k}$,

$$
\pi_{h}\left(f\left(\phi_{h}\right)\right)=f\left(\pi_{h}\left(\phi_{h}\right)\right) .
$$

The proof of Lemma D.4 is straightforward since, by Definition D.2, the interpolation $\pi_{h}$ only uses specific values at fixed points in the spatial domain $\mathcal{D}$.

\section{D.2. Free energy estimates with discontinuous piecewise linear $\sigma_{h}$}

In this section, we consider the following finite element discretization: Scott-Vogelius $\left(\mathbb{P}_{2}\right)^{d} \times \mathbb{P}_{1 \text {,disc }}$ for $\left(\boldsymbol{u}_{h}, p_{h}\right)$ and $\left(\mathbb{P}_{1, \mathrm{disc}}\right)^{\frac{d(d+1)}{2}}$ or $\left(\mathbb{P}_{1}+\mathbb{P}_{0}\right)^{\frac{d(d+1)}{2}}$ for $\boldsymbol{\sigma}_{h}$. 


\section{D.2.1. The characteristic method}

If the advection term $\boldsymbol{u} \cdot \boldsymbol{\nabla} \boldsymbol{\sigma}$ is discretized by the characteristic method, the system writes:

$$
\begin{aligned}
0= & \int_{\mathcal{D}} \operatorname{Re}\left(\frac{\boldsymbol{u}_{h}^{n+1}-\boldsymbol{u}_{h}^{n}}{\Delta t}+\boldsymbol{u}_{h}^{n} \cdot \boldsymbol{\nabla} \boldsymbol{u}_{h}^{n+1}\right) \cdot \boldsymbol{v}-p_{h}^{n+1} \operatorname{div} \boldsymbol{v}+q \operatorname{div} \boldsymbol{u}_{h}^{n+1} \\
& +(1-\varepsilon) \boldsymbol{\nabla} \boldsymbol{u}_{h}^{n+1}: \boldsymbol{\nabla} \boldsymbol{v}+\frac{\varepsilon}{\mathrm{Wi}} \pi_{h}\left(\boldsymbol{\sigma}_{h}^{n+1}\right): \boldsymbol{\nabla} \boldsymbol{v} \\
& +\left(\frac{\boldsymbol{\sigma}_{h}^{n+1}-\pi_{h}\left(\boldsymbol{\sigma}_{h}^{n}\right) \circ X^{n}\left(t^{n}\right)}{\Delta t}\right): \boldsymbol{\phi}-\left(\boldsymbol{\nabla} \boldsymbol{u}_{h}^{n+1} \pi_{h}\left(\boldsymbol{\sigma}_{h}^{n+1}\right)+\pi_{h}\left(\boldsymbol{\sigma}_{h}^{n+1}\right)\left(\boldsymbol{\nabla} \boldsymbol{u}_{h}^{n+1}\right)^{T}\right): \boldsymbol{\phi}+\frac{1}{\mathrm{Wi}}\left(\boldsymbol{\sigma}_{h}^{n+1}-\boldsymbol{I}\right): \boldsymbol{\phi}
\end{aligned}
$$

where $X^{n}$ is defined as in (3.7). Notice that we have used the projection operator $\pi_{h}$ in four terms. It will become clearer from the proof of the free energy estimate below why those projections are needed.

Proposition D.5. Let $\left(\boldsymbol{u}_{h}^{n}, p_{h}^{n}, \boldsymbol{\sigma}_{h}^{n}\right)_{0 \leq n \leq N_{T}}$ be a solution to (D.1), such that $\pi_{h}\left(\boldsymbol{\sigma}_{h}^{n}\right)$ is positive definite. Then, the free energy of the solution $\left(\boldsymbol{u}_{h}^{n}, p_{h}^{n}, \boldsymbol{\sigma}_{h}^{n}\right)$ :

$$
F_{h}^{n}=F\left(\boldsymbol{u}_{h}^{n}, \pi_{h}\left(\boldsymbol{\sigma}_{h}^{n}\right)\right)=\frac{\operatorname{Re}}{2} \int_{\mathcal{D}}\left|\boldsymbol{u}_{h}^{n}\right|^{2}+\frac{\varepsilon}{2 \mathrm{Wi}} \int_{\mathcal{D}} \operatorname{tr}\left(\pi_{h}\left(\boldsymbol{\sigma}_{h}^{n}\right)-\ln \pi_{h}\left(\boldsymbol{\sigma}_{h}^{n}\right)-\boldsymbol{I}\right),
$$

satisfies:

$$
F_{h}^{n+1}-F_{h}^{n}+\int_{\mathcal{D}} \frac{\operatorname{Re}}{2}\left|\boldsymbol{u}_{h}^{n+1}-\boldsymbol{u}_{h}^{n}\right|^{2}+\Delta t \int_{\mathcal{D}}(1-\varepsilon)\left|\nabla \boldsymbol{u}_{h}^{n+1}\right|^{2}+\frac{\varepsilon}{2 \mathrm{Wi}^{2}} \operatorname{tr}\left(\pi_{h}\left(\boldsymbol{\sigma}_{h}^{n+1}\right)+\pi_{h}\left(\boldsymbol{\sigma}_{h}^{n+1}\right)^{-1}-2 \boldsymbol{I}\right) \leq 0
$$

In particular, the sequence $\left(F_{h}^{n}\right)_{0 \leq n \leq N_{T}}$ is non-increasing.

Remark D.6. The ensemble of symmetric positive definite matrices is convex. This implies that a piecewise linear tensor field is symmetric positive definite as soon as it is symmetric positive definite at the nodes of the mesh. Moreover, this also implies that $\pi_{h}\left(\boldsymbol{\sigma}_{h}\right)$ is symmetric positive definite as soon as $\boldsymbol{\sigma}_{h}$ is a piecewise linear (possibly discontinuous) symmetric positive definite tensor field.

Proof of Proposition D.5. The test functions we choose are $\left(\boldsymbol{u}_{h}^{n+1}, p_{h}^{n+1}, \frac{\varepsilon}{2 \mathrm{Wi}}\left(\boldsymbol{I}-\pi_{h}\left(\boldsymbol{\sigma}_{h}^{n+1}\right)^{-1}\right)\right)$. Recall that by Lemma D.4, $\left(\pi_{h}\left(\boldsymbol{\sigma}_{h}^{n+1}\right)\right)^{-1}=\pi_{h}\left(\left(\boldsymbol{\sigma}_{h}^{n+1}\right)^{-1}\right)$. The proof is similar to the one of Proposition 4.1 except in the treatment of the constitutive equation. The upper-convective term in the tensor derivative writes (using Lem. D.3 and the incompressibility property (3.3)):

$$
\begin{aligned}
\int_{\mathcal{D}} \boldsymbol{\nabla} \boldsymbol{u}_{h}^{n+1} \pi_{h}\left(\boldsymbol{\sigma}_{h}^{n+1}\right):\left(\boldsymbol{I}-\pi_{h}\left(\boldsymbol{\sigma}_{h}^{n+1}\right)^{-1}\right) & =\int_{\mathcal{D}} \pi_{h}\left(\boldsymbol{\sigma}_{h}^{n+1}\right): \nabla \boldsymbol{u}_{h}^{n+1}-\int_{\mathcal{D}} \boldsymbol{\nabla} \boldsymbol{u}_{h}^{n+1} \pi_{h}\left(\boldsymbol{\sigma}_{h}^{n+1}\right): \pi_{h}\left(\boldsymbol{\sigma}_{h}^{n+1}\right)^{-1} \\
& =\int_{\mathcal{D}} \pi_{h}\left(\boldsymbol{\sigma}_{h}^{n+1}\right): \nabla \boldsymbol{u}_{h}^{n+1}-\int_{\mathcal{D}} \boldsymbol{\nabla} \boldsymbol{u}_{h}^{n+1}: \boldsymbol{I} \\
& =\int_{\mathcal{D}} \pi_{h}\left(\boldsymbol{\sigma}_{h}^{n+1}\right): \nabla \boldsymbol{u}_{h}^{n+1}-\int_{\mathcal{D}} \operatorname{div} \boldsymbol{u}_{h}^{n+1} \\
& =\int_{\mathcal{D}} \pi_{h}\left(\boldsymbol{\sigma}_{h}^{n+1}\right): \boldsymbol{\nabla} \boldsymbol{u}_{h}^{n+1}
\end{aligned}
$$

which vanishes after combination with the extra-stress term in the momentum equation.

The last term rewrites (using again Lem. D.3):

$$
\int_{\mathcal{D}}\left(\boldsymbol{\sigma}_{h}^{n+1}-\boldsymbol{I}\right):\left(\boldsymbol{I}-\pi_{h}\left(\boldsymbol{\sigma}_{h}^{n+1}\right)^{-1}\right)=\int_{\mathcal{D}} \operatorname{tr}\left(\pi_{h}\left(\boldsymbol{\sigma}_{h}^{n+1}\right)+\pi_{h}\left(\boldsymbol{\sigma}_{h}^{n+1}\right)^{-1}-2 \boldsymbol{I}\right)
$$


The remaining term writes (using Lem. D.3, Eq. (1.10) with $\boldsymbol{\sigma}=\pi_{h}\left(\boldsymbol{\sigma}_{h}^{n}\right) \circ X^{n}\left(t^{n}\right)$ and $\boldsymbol{\tau}=\pi_{h}\left(\boldsymbol{\sigma}_{h}^{n+1}\right)$, and the fact that the Jacobian of $X^{n}$ remains equal to one due to the incompressibility property (3.3)):

$$
\begin{aligned}
\int_{\mathcal{D}} & \left(\boldsymbol{\sigma}_{h}^{n+1}-\pi_{h}\left(\boldsymbol{\sigma}_{h}^{n}\right) \circ X^{n}\left(t^{n}\right)\right):\left(\boldsymbol{I}-\pi_{h}\left(\boldsymbol{\sigma}_{h}^{n+1}\right)^{-1}\right)=\int_{\mathcal{D}} \operatorname{tr} \boldsymbol{\sigma}_{h}^{n+1}-\operatorname{tr} \pi_{h}\left(\boldsymbol{\sigma}_{h}^{n}\right) \circ X^{n}\left(t^{n}\right) \\
& +\operatorname{tr}\left(\pi_{h}\left(\boldsymbol{\sigma}_{h}^{n}\right) \circ X^{n}\left(t^{n}\right) \pi_{h}\left(\boldsymbol{\sigma}_{h}^{n+1}\right)^{-1}-\boldsymbol{I}\right) \\
& \geq \int_{\mathcal{D}} \operatorname{tr} \boldsymbol{\sigma}_{h}^{n+1}-\operatorname{tr} \pi_{h}\left(\boldsymbol{\sigma}_{h}^{n}\right) \circ X^{n}\left(t^{n}\right)+\operatorname{tr} \ln \pi_{h}\left(\boldsymbol{\sigma}_{h}^{n}\right) \circ X^{n}\left(t^{n}\right)-\operatorname{tr} \ln \pi_{h}\left(\boldsymbol{\sigma}_{h}^{n+1}\right) \\
& =\int_{\mathcal{D}} \operatorname{tr} \pi_{h}\left(\boldsymbol{\sigma}_{h}^{n+1}\right)-\operatorname{tr} \pi_{h}\left(\boldsymbol{\sigma}_{h}^{n}\right)+\operatorname{tr} \ln \pi_{h}\left(\boldsymbol{\sigma}_{h}^{n}\right)-\operatorname{tr} \ln \pi_{h}\left(\boldsymbol{\sigma}_{h}^{n+1}\right) .
\end{aligned}
$$

This completes the proof.

\section{D.2.2. The discontinuous Galerkin method}

If the advection term $\boldsymbol{u} \cdot \boldsymbol{\nabla} \boldsymbol{\sigma}$ is discretized by the discontinuous Galerkin method, the system writes:

$$
\begin{aligned}
0=\sum_{k=1}^{N_{K}} \int_{K_{k}} \operatorname{Re}\left(\frac{\boldsymbol{u}_{h}^{n+1}-\boldsymbol{u}_{h}^{n}}{\Delta t}+\boldsymbol{u}_{h}^{n} \cdot \boldsymbol{\nabla} \boldsymbol{u}_{h}^{n+1}\right) \cdot \boldsymbol{v}-p_{h}^{n+1} \operatorname{div} \boldsymbol{v}+q \operatorname{div} \boldsymbol{u}_{h}^{n+1} \\
\quad+(1-\varepsilon) \boldsymbol{\nabla} \boldsymbol{u}_{h}^{n+1}: \boldsymbol{\nabla} \boldsymbol{v}+\frac{\varepsilon}{\mathrm{Wi}} \pi_{h}\left(\boldsymbol{\sigma}_{h}^{n+1}\right): \boldsymbol{\nabla} \boldsymbol{v} \\
+\left(\frac{\boldsymbol{\sigma}_{h}^{n+1}-\boldsymbol{\sigma}_{h}^{n}}{\Delta t}\right): \boldsymbol{\phi}-\left(\boldsymbol{\nabla} \boldsymbol{u}_{h}^{n+1} \pi_{h}\left(\boldsymbol{\sigma}_{h}^{n+1}\right)+\pi_{h}\left(\boldsymbol{\sigma}_{h}^{n+1}\right)\left(\boldsymbol{\nabla} \boldsymbol{u}_{h}^{n+1}\right)^{T}\right): \boldsymbol{\phi}+\frac{1}{\mathrm{Wi}}\left(\boldsymbol{\sigma}_{h}^{n+1}-\boldsymbol{I}\right): \boldsymbol{\phi} \\
\quad+\sum_{j=1}^{N_{E}} \int_{E_{j}}\left|\boldsymbol{u}_{h}^{n} \cdot \boldsymbol{n}\right| \llbracket \pi_{h}\left(\boldsymbol{\sigma}_{h}^{n+1}\right) \rrbracket: \boldsymbol{\phi}^{+} .
\end{aligned}
$$

As for the characteristic method, the projection operator $\pi_{h}$ is used in four terms. Besides, like in the case where $\sigma_{h} \in\left(\mathbb{P}_{0}\right)^{\frac{d(d+1)}{2}}$, the advection term $\boldsymbol{u} \cdot \nabla \boldsymbol{\sigma}$ is discretized using a jump term only. Indeed, in order to derive discrete free energy estimates, we treat the discrete advection term using the projection $\pi_{h}\left(\boldsymbol{\sigma}_{h}\right) \in\left(\mathbb{P}_{0}\right)^{\frac{d(d+1)}{2}}$ of the stress field $\boldsymbol{\sigma}_{h}$, the derivative of which is zero.

Proposition D.5 still holds for the system (D.4). The proof is straightforward using all the arguments of the previous sections, except for the treatment of the discrete advection term for $\boldsymbol{u} \cdot \boldsymbol{\nabla} \boldsymbol{\sigma}$. Using equations (1.10), (3.5), the fact that $\pi_{h}\left(\boldsymbol{\sigma}_{h}^{n+1}\right) \in\left(\mathbb{P}_{0}\right)^{\frac{d(d+1)}{2}}$ and the weak incompressibility property (4.7), we have:

$$
\begin{aligned}
\sum_{j=1}^{N_{E}} \int_{E_{j}}\left|\boldsymbol{u}_{h}^{n} \cdot \boldsymbol{n}\right| \llbracket \pi_{h}\left(\boldsymbol{\sigma}_{h}^{n+1}\right) \rrbracket\left(\boldsymbol{I}-\pi_{h}\left(\boldsymbol{\sigma}_{h}^{n+1}\right)^{-1}\right)^{+}= \\
\quad \sum_{j=1}^{N_{E}} \int_{E_{j}}\left|\boldsymbol{u}_{h}^{n} \cdot \boldsymbol{n}\right| \llbracket \operatorname{tr} \pi_{h}\left(\boldsymbol{\sigma}_{h}^{n+1}\right) \rrbracket+\left|\boldsymbol{u}_{h}^{n} \cdot \boldsymbol{n}\right| \operatorname{tr}\left(\pi_{h}\left(\boldsymbol{\sigma}_{h}^{n+1,-}\right) \pi_{h}\left(\boldsymbol{\sigma}_{h}^{n+1,+}\right)^{-1}-\boldsymbol{I}\right) \\
\geq \sum_{j=1}^{N_{E}} \int_{E_{j}}\left|\boldsymbol{u}_{h}^{n} \cdot \boldsymbol{n}\right| \llbracket \operatorname{tr} \pi_{h}\left(\boldsymbol{\sigma}_{h}^{n+1}\right) \rrbracket+\left|\boldsymbol{u}_{h}^{n} \cdot \boldsymbol{n}\right| \operatorname{tr}\left(\ln \pi_{h}\left(\boldsymbol{\sigma}_{h}^{n+1,-}\right)-\ln \pi_{h}\left(\boldsymbol{\sigma}_{h}^{n+1,+}\right)\right) .
\end{aligned}
$$


Now, the right-hand-side vanishes since it is equal to

$$
\begin{aligned}
\sum_{j=1}^{N_{E}} \int_{E_{j}}\left|\boldsymbol{u}_{h}^{n} \cdot \boldsymbol{n}\right| \llbracket \operatorname{tr}\left(\pi_{h}\left(\boldsymbol{\sigma}_{h}^{n+1}\right)-\ln \pi_{h}\left(\boldsymbol{\sigma}_{h}^{n+1}\right)\right) \rrbracket & =\sum_{k=1}^{N_{K}}-\int_{\partial K_{k}}\left(\boldsymbol{u}_{h}^{n} \cdot \boldsymbol{n}_{K_{k}}\right) \operatorname{tr}\left(\pi_{h}\left(\boldsymbol{\sigma}_{h}^{n+1}\right)-\ln \pi_{h}\left(\boldsymbol{\sigma}_{h}^{n+1}\right)\right) \\
& =\sum_{k=1}^{N_{K}}-\left.\operatorname{tr}\left(\pi_{h}\left(\boldsymbol{\sigma}_{h}^{n+1}\right)-\ln \pi_{h}\left(\boldsymbol{\sigma}_{h}^{n+1}\right)\right)\right|_{K_{k}} \int_{K_{k}} \operatorname{div}\left(\boldsymbol{u}_{h}^{n}\right)=0 .
\end{aligned}
$$

\section{D.3. Free energy estimates with discontinuous piecewise linear $\psi_{h}$}

In the following section, we write free-energy-dissipative schemes using the log-formulation with $\boldsymbol{\psi}_{h}$ piecewise linear. For this, we again need the projection operator $\pi_{h}$ introduced in Definition D.2. We consider the ScottVogelius finite element space for $\left(\boldsymbol{u}_{h}, p_{h}\right)$ and the following decomposition of the velocity gradient $\boldsymbol{\nabla} \boldsymbol{u}_{h} \in$ $\left(\mathbb{P}_{1, \text { disc }}\right)^{\frac{d(d+1)}{2}}$ :

$$
\nabla \boldsymbol{u}_{h}=\boldsymbol{\Omega}_{h}+\boldsymbol{B}_{h}+\boldsymbol{N}_{h} \pi_{h}\left(\mathrm{e}^{\psi_{h}}\right)^{-1} .
$$

Notice that since $\pi_{h}\left(\mathrm{e}^{\boldsymbol{\psi}_{h}}\right)^{-1}=\mathrm{e}^{-\pi_{h}\left(\boldsymbol{\psi}_{h}\right)}$ is in $\left(\mathbb{P}_{0}\right)^{\frac{d(d+1)}{2}}$, we have $\boldsymbol{\Omega}_{h}, \boldsymbol{B}_{h}, \boldsymbol{N}_{h} \in\left(\mathbb{P}_{1, \text { disc }}\right)^{\frac{d(d+1)}{2}}$ by virtue of Lemma 3.3 with $k=1$.

\section{D.3.1. The characteristic method}

If the advection term $\boldsymbol{u} \cdot \boldsymbol{\nabla} \boldsymbol{\sigma}$ is discretized by the characteristic method, the system writes:

$$
\begin{array}{r}
0=\int_{\mathcal{D}} \operatorname{Re}\left(\frac{\boldsymbol{u}_{h}^{n+1}-\boldsymbol{u}_{h}^{n}}{\Delta t}+\boldsymbol{u}_{h}^{n} \cdot \boldsymbol{\nabla} \boldsymbol{u}_{h}^{n+1}\right) \cdot \boldsymbol{v}-p_{h}^{n+1} \operatorname{div} \boldsymbol{v}+q \operatorname{div} \boldsymbol{u}_{h}^{n+1}+(1-\varepsilon) \boldsymbol{\nabla} \boldsymbol{u}_{h}^{n+1}: \nabla \boldsymbol{v}+\frac{\varepsilon}{\mathrm{Wi}} \pi_{h}\left(\mathrm{e}^{\boldsymbol{\psi}_{h}^{n+1}}\right): \boldsymbol{\nabla} \boldsymbol{v} \\
+\left(\frac{\boldsymbol{\psi}_{h}^{n+1}-\pi_{h}\left(\boldsymbol{\psi}_{h}^{n}\right) \circ X^{n}\left(t^{n}\right)}{\Delta t}\right): \boldsymbol{\phi}-\left(\boldsymbol{\Omega}_{h}^{n+1} \pi_{h}\left(\boldsymbol{\psi}_{h}^{n+1}\right)-\pi_{h}\left(\boldsymbol{\psi}_{h}^{n+1}\right) \boldsymbol{\Omega}_{h}^{n+1}\right): \boldsymbol{\phi} \\
-2 \boldsymbol{B}_{h}^{n+1}: \boldsymbol{\phi}-\frac{1}{\mathrm{Wi}}\left(\pi_{h}\left(\mathrm{e}^{-\boldsymbol{\psi}_{h}^{n+1}}\right)-\boldsymbol{I}\right): \boldsymbol{\phi} . \quad(\mathrm{D} .6)
\end{array}
$$

In the system above, we have used the projection operator $\pi_{h}$ to treat the same terms as in the system (D.1). But in addition to these, we have also used the projection operator for the exponential term $\mathrm{e}^{-\boldsymbol{\psi}_{h}^{n+1}}$ in the Oldroyd-B equation.

Proposition D.7. Let $\left(\boldsymbol{u}_{h}^{n}, p_{h}^{n}, \boldsymbol{\psi}_{h}^{n}\right)_{0 \leq n \leq N_{T}}$ be a solution to (D.6). Then, the free energy of the solution $\left(\boldsymbol{u}_{h}^{n}, p_{h}^{n}, \boldsymbol{\psi}_{h}^{n}\right)$ :

$$
F_{h}^{n}=F\left(\boldsymbol{u}_{h}^{n}, \mathrm{e}^{\pi_{h}\left(\boldsymbol{\psi}_{h}^{n}\right)}\right)=\frac{\operatorname{Re}}{2} \int_{\mathcal{D}}\left|\boldsymbol{u}_{h}\right|^{2}+\frac{\varepsilon}{2 \mathrm{Wi}} \int_{\mathcal{D}} \operatorname{tr}\left(\mathrm{e}^{\pi_{h}\left(\boldsymbol{\psi}_{h}^{n}\right)}-\pi_{h}\left(\boldsymbol{\psi}_{h}^{n}\right)-\boldsymbol{I}\right)
$$

satisfies:

$$
F_{h}^{n+1}-F_{h}^{n}+\int_{\mathcal{D}} \frac{\operatorname{Re}}{2}\left|\boldsymbol{u}_{h}^{n+1}-\boldsymbol{u}_{h}^{n}\right|^{2}+\Delta t \int_{\mathcal{D}}(1-\varepsilon)\left|\nabla \boldsymbol{u}_{h}^{n+1}\right|^{2}+\frac{\varepsilon}{2 \mathrm{Wi}^{2}} \operatorname{tr}\left(\mathrm{e}^{\pi_{h}\left(\boldsymbol{\psi}_{h}^{n}\right)}+\mathrm{e}^{-\pi_{h}\left(\boldsymbol{\psi}_{h}^{n}\right)}-2 \boldsymbol{I}\right) \leq 0
$$

In particular, the sequence $\left(F_{h}^{n}\right)_{0 \leq n \leq N_{T}}$ is non-increasing.

Proof of Proposition D.7. The proof is similar to that of Proposition 4.3 except for the terms using the interpolation operator $\pi_{h}$. We shall use as test functions $\left(\boldsymbol{u}_{h}^{n+1}, p_{h}^{n+1}, \frac{\varepsilon}{2 \mathrm{Wi}}\left(\pi_{h}\left(\mathrm{e}^{\boldsymbol{\psi}_{h}^{n+1}}\right)-\boldsymbol{I}\right)\right)$ in (3.10). Also, we will make use of the following property throughout the proof (see Lem. D.4): $\pi_{h}\left(\mathrm{e}^{\boldsymbol{\psi}_{h}^{n+1}}\right)=\mathrm{e}^{\pi_{h}\left(\boldsymbol{\psi}_{h}^{n+1}\right)}$. 
For the material derivative of $\boldsymbol{\psi}_{h}$, using Lemma D.3, equation (1.11) with $\boldsymbol{\sigma}=\mathrm{e}_{h}^{\boldsymbol{\psi}_{h}^{n+1}}$ and $\boldsymbol{\tau}=\mathrm{e}^{\boldsymbol{\psi}_{h}^{n} \circ X^{n}\left(t^{n}\right)}$, and the fact that the Jacobian of the flow $X^{n}$ is one for divergence-free velocity field $\boldsymbol{u}_{h}^{n}$, we have:

$$
\begin{aligned}
& \int_{\mathcal{D}}\left(\boldsymbol{\psi}_{h}^{n+1}-\pi_{h}\left(\boldsymbol{\psi}_{h}^{n}\right) \circ X^{n}\left(t^{n}\right)\right):\left(\pi_{h}\left(\mathrm{e}^{\boldsymbol{\psi}_{h}^{n+1}}\right)-\boldsymbol{I}\right)=\int_{\mathcal{D}}\left(\pi_{h}\left(\boldsymbol{\psi}_{h}^{n+1}\right)-\pi_{h}\left(\boldsymbol{\psi}_{h}^{n}\right) \circ X^{n}\left(t^{n}\right)\right): \mathrm{e}^{\pi_{h}\left(\boldsymbol{\psi}_{h}^{n+1}\right)} \\
&-\operatorname{tr}\left(\pi_{h}\left(\boldsymbol{\psi}_{h}^{n+1}\right)-\pi_{h}\left(\boldsymbol{\psi}_{h}^{n}\right) \circ X^{n}\left(t^{n}\right)\right) \\
& \geq \int_{\mathcal{D}} \operatorname{tr}\left(\mathrm{e}^{\pi_{h}\left(\boldsymbol{\psi}_{h}^{n+1}\right)}-\pi_{h}\left(\boldsymbol{\psi}_{h}^{n+1}\right)\right)-\int_{\mathcal{D}} \operatorname{tr}\left(\mathrm{e}^{\pi_{h}\left(\boldsymbol{\psi}_{h}^{n}\right)}-\pi_{h}\left(\boldsymbol{\psi}_{h}^{n}\right)\right) \circ X^{n}\left(t^{n}\right) \\
&=\int_{\mathcal{D}} \operatorname{tr}\left(\mathrm{e}^{\pi_{h}\left(\boldsymbol{\psi}_{h}^{n+1}\right)}-\pi_{h}\left(\boldsymbol{\psi}_{h}^{n+1}\right)\right)-\int_{\mathcal{D}} \operatorname{tr}\left(\mathrm{e}^{\pi_{h}\left(\boldsymbol{\psi}_{h}^{n}\right)}-\pi_{h}\left(\boldsymbol{\psi}_{h}^{n}\right)\right) .
\end{aligned}
$$

Besides, using equation (2.16), we have:

$$
\begin{aligned}
\int_{\mathcal{D}}\left(\boldsymbol{\Omega}_{h}^{n+1} \pi_{h}\left(\boldsymbol{\psi}_{h}^{n+1}\right)-\pi_{h}\left(\boldsymbol{\psi}_{h}^{n+1}\right) \boldsymbol{\Omega}_{h}^{n+1}\right):\left(\mathrm{e}^{\pi_{h}\left(\boldsymbol{\psi}_{h}^{n+1}\right)}-\boldsymbol{I}\right)= \\
\qquad \int_{\mathcal{D}}\left(\boldsymbol{\Omega}_{h}^{n+1} \pi_{h}\left(\boldsymbol{\psi}_{h}^{n+1}\right)-\pi_{h}\left(\boldsymbol{\psi}_{h}^{n+1}\right) \boldsymbol{\Omega}_{h}^{n+1}\right): \mathrm{e}^{\pi_{h}\left(\boldsymbol{\psi}_{h}^{n+1}\right)}=0,
\end{aligned}
$$

and using equations (2.15) and (3.3):

$$
\int_{\mathcal{D}} \boldsymbol{B}_{h}^{n+1}:\left(\pi_{h}\left(\mathrm{e}^{\boldsymbol{\psi}_{h}^{n+1}}\right)-\boldsymbol{I}\right)=\int_{\mathcal{D}} \boldsymbol{B}_{h}^{n+1}: \mathrm{e}^{\pi_{h}\left(\boldsymbol{\psi}_{h}^{n+1}\right)}-\int_{\mathcal{D}} \operatorname{div}\left(\boldsymbol{u}_{h}^{n+1}\right)=\int_{\mathcal{D}} \boldsymbol{\nabla} \boldsymbol{u}_{h}^{n+1}: \mathrm{e}^{\pi_{h}\left(\boldsymbol{\psi}_{h}^{n+1}\right)},
$$

which cancels out with the same term $\int_{\mathcal{D}} \mathrm{e}^{\pi_{h}\left(\psi_{h}^{n+1}\right)}: \nabla \boldsymbol{u}_{h}^{n+1}$ in the momentum equation.

\section{D.3.2. The discontinuous Galerkin method}

If the advection term $\boldsymbol{u} \cdot \boldsymbol{\nabla} \boldsymbol{\sigma}$ is discretized by the discontinuous Galerkin method, the system writes:

$$
\begin{aligned}
& 0= \sum_{k=1}^{N_{K}} \int_{K_{k}} \operatorname{Re}\left(\frac{\boldsymbol{u}_{h}^{n+1}-\boldsymbol{u}_{h}^{n}}{\Delta t}+\boldsymbol{u}_{h}^{n} \cdot \boldsymbol{\nabla} \boldsymbol{u}_{h}^{n+1}\right) \cdot \boldsymbol{v}-p_{h}^{n+1} \operatorname{div} \boldsymbol{v}+q \operatorname{div} \boldsymbol{u}_{h}^{n+1}+(1-\varepsilon) \boldsymbol{\nabla} \boldsymbol{u}_{h}^{n+1}: \boldsymbol{\nabla} \boldsymbol{v} \\
&+\frac{\varepsilon}{\mathrm{Wi}} \pi_{h}\left(\mathrm{e}^{\psi_{h}^{n+1}}\right): \boldsymbol{\nabla} \boldsymbol{v}\left(\frac{\boldsymbol{\psi}_{h}^{n+1}-\pi_{h}\left(\boldsymbol{\psi}_{h}^{n}\right)}{\Delta t}\right): \boldsymbol{\phi}-\left(\boldsymbol{\Omega}_{h}^{n+1} \pi_{h}\left(\boldsymbol{\psi}_{h}^{n+1}\right)-\pi_{h}\left(\boldsymbol{\psi}_{h}^{n+1}\right) \boldsymbol{\Omega}_{h}^{n+1}\right): \boldsymbol{\phi}-2 \boldsymbol{B}_{h}^{n+1}: \boldsymbol{\phi} \\
& \quad-\frac{1}{\mathrm{Wi}}\left(\pi_{h}\left(\mathrm{e}^{-\boldsymbol{\psi}_{h}^{n+1}}\right)-\boldsymbol{I}\right): \boldsymbol{\phi}+\sum_{j=1}^{N_{E}} \int_{E_{j}}\left|\boldsymbol{u}_{h}^{n} \cdot \boldsymbol{n}\right| \llbracket \pi_{h}\left(\boldsymbol{\psi}_{h}^{n+1}\right) \rrbracket: \boldsymbol{\phi}^{+} . \quad(\mathrm{D} .9)
\end{aligned}
$$

Proposition D.7 still holds for solutions of the system (D.9). The proof follows that of the previous Section D.3.1 except for the treatment of the jump term, which follows that of Section 4.1.2 (see also Sect. 4.2.2), because $\pi_{h}\left(\boldsymbol{\psi}_{h}^{n+1}\right) \in\left(\mathbb{P}_{0}\right)^{\frac{d(d+1)}{2}}$ and $\pi_{h}\left(\mathrm{e}^{\boldsymbol{\psi}_{h}^{n+1}}\right)=\mathrm{e}^{\pi_{h}\left(\psi_{h}^{n+1}\right)}$ is also in $\left(\mathbb{P}_{0}\right)^{\frac{d(d+1)}{2}}$.

\section{D.4. Other finite elements for $\left(u_{h}, p_{h}\right)$}

In this section, we review the modifications that apply to the systems in the two previous Sections D.2 and D.3 when the different mixed finite element spaces for $\left(\boldsymbol{u}_{h}, p_{h}\right)$ proposed in Section 4.3 are used instead of Scott-Vogelius. Notice that the conclusions of Table 1 about the conditions that the velocity field has to satisfy still hold for the two previous Sections D.2 and D.3 with piecewise linear approximations of $\boldsymbol{\sigma}_{h}, \boldsymbol{\psi}_{h}$.

Other finite elements space for $\left(\boldsymbol{u}_{h}, p_{h}\right)$ than Scott-Vogelius and adequate projections of the velocity field (see summary in Tab. 2) have to be combined with interpolations of the stress field $\boldsymbol{\sigma}_{h}, \boldsymbol{\psi}_{h}$ using $\pi_{h}$ (see the two previous Sects. D.2 and D.3 above). We give a summary of the projections that are required in Table 3. 


\section{D.4.1. Alternative mixed finite element space for $\left(\boldsymbol{u}_{h}, p_{h}\right)$ with inf-sup condition}

The situation is very similar to that in Section 4.3.2. Among the mixed finite element space that satisfy the inf-sup condition, let us first choose the Taylor-Hood $\left(\mathbb{P}_{2}\right)^{d} \times \mathbb{P}_{1}$. Again, because the velocity is not even weakly incompressible in the sense of equation (4.7), we need to use the projection of the velocity field onto the solenoidal vector fields for the treatment of some terms in the variational formulations. When the advection terms $\boldsymbol{u} \cdot \nabla \boldsymbol{\sigma}$ and $\boldsymbol{u} \cdot \boldsymbol{\nabla} \boldsymbol{\psi}$ are discretized using the characteristic method, we define the flow with $P_{h}^{\text {rot }}\left(\boldsymbol{u}_{h}^{n}\right)$ like in (4.10) and use the same systems (D.1) and (D.6) as above. When the advection terms $\boldsymbol{u} \cdot \boldsymbol{\nabla} \boldsymbol{\sigma}$ and $\boldsymbol{u} \cdot \boldsymbol{\nabla} \boldsymbol{\psi}$ are discretized using the discontinuous Galerkin method, we use systems similar to (D.1) and (D.6) above, where the jump term rewrites (in the conformation-tensor formulation):

$$
+\sum_{j=1}^{N_{E}} \int_{E_{j}}\left|P_{h}^{\text {rot }}\left(\boldsymbol{u}_{h}^{n}\right) \cdot \boldsymbol{n}\right| \llbracket \pi_{h}\left(\boldsymbol{\sigma}_{h}^{n+1}\right) \rrbracket: \boldsymbol{\phi}^{+} .
$$

Also, one still needs to add the so-called Temam correction term (4.11) to the weak formulation.

We can also use the Crouzeix-Raviart finite elements for velocity (see (4.8)): $\left(\boldsymbol{u}_{h}, p_{h}, \boldsymbol{\sigma}_{h}\right)$ in $\left(\mathbb{P}_{1}^{C R}\right)^{d} \times \mathbb{P}_{0} \times$ $\left(\mathbb{P}_{1, \text { disc }}\right)^{\frac{d(d+1)}{2}}$. Similarly to the advection terms $\boldsymbol{u} \cdot \nabla \boldsymbol{\sigma}$ and $\boldsymbol{u} \cdot \boldsymbol{\nabla} \boldsymbol{\psi}$, the advection term $\boldsymbol{u} \cdot \boldsymbol{\nabla} \boldsymbol{u}$ in the NavierStokes equations should then be discretized either using a characteristic method with the flow defined in (4.13) with any of the projections $P_{h}$ introduced above for the velocity field, or using the discontinuous Galerkin method formulated in equation (4.14).

It is noticeable that choosing the mixed finite elements of Crouzeix-Raviart simplifies all the variational formulations presented above in the present Section D. Indeed, since $\boldsymbol{\nabla} \boldsymbol{u} \in\left(\mathbb{P}_{0}\right)^{d \times d}$ and we have the Lemma D.3, it is then unnecessary to project the velocity except in the advection terms. For instance, for the conformationtensor formulation using the discontinuous Galerkin method, the formulation writes:

$$
\begin{aligned}
0=\sum_{k=1}^{N_{K}} \int_{K_{k}} \operatorname{Re}\left(\frac{\boldsymbol{u}_{h}^{n+1}-\boldsymbol{u}_{h}^{n}}{\Delta t}+P_{h}\left(\boldsymbol{u}_{h}^{n}\right) \cdot \boldsymbol{\nabla} \boldsymbol{u}_{h}^{n+1}\right) \cdot \boldsymbol{v}-p_{h}^{n+1} \operatorname{div} \boldsymbol{v}+q \operatorname{div} \boldsymbol{u}_{h}^{n+1}+(1-\varepsilon) \boldsymbol{\nabla} \boldsymbol{u}_{h}^{n+1}: \nabla \boldsymbol{v} \\
\quad+\frac{\varepsilon}{\mathrm{Wi}} \boldsymbol{\sigma}_{h}^{n+1}: \boldsymbol{\nabla} \boldsymbol{v}+\left(\frac{\boldsymbol{\sigma}_{h}^{n+1}-\pi_{h}\left(\boldsymbol{\sigma}_{h}^{n}\right)}{\Delta t}\right): \boldsymbol{\phi}-\left(\left(\boldsymbol{\nabla} \boldsymbol{u}_{h}^{n+1}\right) \boldsymbol{\sigma}_{h}^{n+1}+\boldsymbol{\sigma}_{h}^{n+1}\left(\boldsymbol{\nabla} \boldsymbol{u}_{h}^{n+1}\right)^{T}\right): \boldsymbol{\phi} \\
+\frac{1}{\mathrm{Wi}}\left(\boldsymbol{\sigma}_{h}^{n+1}-\boldsymbol{I}\right): \boldsymbol{\phi}+\sum_{j=1}^{N_{E}} \int_{E_{j}}\left|P_{h}\left(\boldsymbol{u}_{h}^{n}\right) \cdot \boldsymbol{n}\right| \llbracket \pi_{h}\left(\boldsymbol{\sigma}_{h}^{n+1}\right) \rrbracket: \boldsymbol{\phi}^{+}+\operatorname{Re}\left|P_{h}\left(\boldsymbol{u}_{h}^{n}\right) \cdot \boldsymbol{n}\right| \llbracket \boldsymbol{u}_{h}^{n+1} \rrbracket \cdot\{\boldsymbol{v}\}
\end{aligned}
$$

Note that the second term in the sum of integrals over edges $E_{j}$ is due to the use of the Crouzeix-Raviart element, and is uncorrelated to the treatment of the advection by a discontinuous Galerkin method.

The discrete free energy estimate (D.3) holds. Its proof combines arguments of the proofs above, except for the treatment of the upper-convective term in (D.10). This term writes, on any element $K_{k}$ of the mesh (using Lem. D.3, the fact that $\boldsymbol{\nabla} \boldsymbol{u} \in\left(\mathbb{P}_{0}\right)^{d \times d}$ and the incompressibility (3.3)):

$$
\begin{aligned}
\int_{K_{k}} \boldsymbol{\nabla} \boldsymbol{u}_{h}^{n+1} \boldsymbol{\sigma}_{h}^{n+1}:\left(\boldsymbol{I}-\pi_{h}\left(\boldsymbol{\sigma}_{h}^{n+1}\right)^{-1}\right) & =\int_{K_{k}} \boldsymbol{\sigma}_{h}^{n+1}: \nabla \boldsymbol{u}_{h}^{n+1}-\int_{\mathcal{D}} \boldsymbol{\sigma}_{h}^{n+1}: \pi_{h}\left(\boldsymbol{\sigma}_{h}^{n+1}\right)^{-1} \nabla \boldsymbol{u}_{h}^{n+1} \\
& =\int_{K_{k}} \pi_{h}\left(\boldsymbol{\sigma}_{h}^{n+1}\right): \nabla \boldsymbol{u}_{h}^{n+1}-\int_{\mathcal{D}} \pi_{h}\left(\boldsymbol{\sigma}_{h}^{n+1}\right): \pi_{h}\left(\boldsymbol{\sigma}_{h}^{n+1}\right)^{-1} \nabla \boldsymbol{u}_{h}^{n+1} \\
& =\int_{K_{k}} \pi_{h}\left(\boldsymbol{\sigma}_{h}^{n+1}\right): \nabla \boldsymbol{u}_{h}^{n+1}-\int_{\mathcal{D}} \operatorname{div} \boldsymbol{u}_{h}^{n+1} \\
& =\int_{K_{k}} \pi_{h}\left(\boldsymbol{\sigma}_{h}^{n+1}\right): \nabla \boldsymbol{u}_{h}^{n+1}
\end{aligned}
$$


which vanishes after combination with the extra-stress term in the momentum equation, the latter satisfying:

$$
\int_{K_{k}} \boldsymbol{\sigma}_{h}^{n+1}: \nabla \boldsymbol{u}_{h}^{n+1}=\int_{K_{k}} \pi_{h}\left(\boldsymbol{\sigma}_{h}^{n+1}\right): \nabla \boldsymbol{u}_{h}^{n+1},
$$

because of the fact that $\nabla \boldsymbol{u} \in\left(\mathbb{P}_{0}\right)^{d \times d}$ and using Lemma D.3.

\section{D.4.2. Alternative mixed finite element space for $\left(\boldsymbol{u}_{h}, p_{h}\right)$ without inf-sup}

It is also possible to use finite element spaces for $\left(\boldsymbol{u}_{h}, p_{h}\right)$ that do not satisfy the inf-sup condition like in Section 4.3.3, while the stress field is discretized using discontinuous piecewise linear approximations. The construction of systems of equations and the derivation of discrete free energy estimates then directly follow from the combination of results from Section 4.3.3 with those used above in Section D, after upgrading the degree of the polynomial approximations for the stress field.

If we consider the mixed finite element space $\left(\mathbb{P}_{1}\right)^{d} \times \mathbb{P}_{0}$ for $\left(\boldsymbol{u}_{h}, p_{h}\right)$, and if the term $\boldsymbol{u} \cdot \boldsymbol{\nabla} \boldsymbol{\sigma}$ is discretized with the characteristic method, the system then writes:

$$
\begin{aligned}
0=\sum_{k=1}^{N_{K}} \int_{K_{k}} \operatorname{Re} & \left(\frac{\boldsymbol{u}_{h}^{n+1}-\boldsymbol{u}_{h}^{n}}{\Delta t}+\boldsymbol{u}_{h}^{n} \cdot \boldsymbol{\nabla} \boldsymbol{u}_{h}^{n+1}\right) \cdot \boldsymbol{v}+\frac{\operatorname{Re}}{2} \operatorname{div} \boldsymbol{u}_{h}^{n}\left(\boldsymbol{v} \cdot \boldsymbol{u}_{h}^{n+1}\right)+(1-\varepsilon) \boldsymbol{\nabla} \boldsymbol{u}_{h}^{n+1}: \boldsymbol{\nabla} \boldsymbol{v} \\
& -p_{h}^{n+1} \operatorname{div} \boldsymbol{v}+q \operatorname{div} \boldsymbol{u}_{h}^{n+1}+\frac{\varepsilon}{\mathrm{Wi}} \boldsymbol{\sigma}_{h}^{n+1}: \boldsymbol{\nabla} \boldsymbol{v}+\left(\frac{\boldsymbol{\sigma}_{h}^{n+1}-\pi_{h}\left(\boldsymbol{\sigma}_{h}^{n}\right) \circ X^{n}\left(t^{n}\right)}{\Delta t}\right): \boldsymbol{\phi} \\
& -\left(\left(\boldsymbol{\nabla} \boldsymbol{u}_{h}^{n+1}\right) \boldsymbol{\sigma}_{h}^{n+1}+\boldsymbol{\sigma}_{h}^{n+1}\left(\boldsymbol{\nabla} \boldsymbol{u}_{h}^{n+1}\right)^{T}\right): \boldsymbol{\phi}+\frac{1}{\mathrm{Wi}}\left(\boldsymbol{\sigma}_{h}^{n+1}-\boldsymbol{I}\right): \boldsymbol{\phi}+\sum_{j=1}^{N_{E}}\left|E_{j}\right| \int_{E_{j}} \llbracket p_{h} \rrbracket \llbracket q \rrbracket
\end{aligned}
$$

with a flow $X^{n}$ computed with the projected field $P_{h}^{\text {rot }}\left(\boldsymbol{u}_{h}^{n}\right)$ through (4.10). It is noteworthy that, for the same reason as above in equation (D.10), the projection operator $\pi_{h}$ is needed only for the discretization of the advection term $\boldsymbol{u} \cdot \boldsymbol{\nabla} \boldsymbol{\sigma}$.

If we consider the mixed finite element space $\left(\mathbb{P}_{1}\right)^{d} \times \mathbb{P}_{1}$ for $\left(\boldsymbol{u}_{h}, p_{h}\right)$, it is straightforward to rewrite the system (4.16) where the stress field was only piecewise constant, while using the same argument as above to see that only the advection term for the stress field needs a projected velocity.

Remark D.8. We were not able to establish discrete free energy estimates without interpolating some terms in the formulations above thanks to the operator $\pi_{h}$. This operator projects the stress $\boldsymbol{\sigma}_{h}$ (or $\left.\boldsymbol{\psi}_{h}\right)$ onto $\left(\mathbb{P}_{0}\right)^{\frac{d(d+1)}{2}}$. Thus, for the formulations we have considered in this section, the interest of using larger dimensional spaces for $\sigma_{h}$ (or $\boldsymbol{\psi}_{h}$ ) than $\left(\mathbb{P}_{0}\right)^{\frac{d(d+1)}{2}}$ is not clear. Our aim in this section is simply to exhibit discrete formulations with piecewise linear approximations of the stress, for which we are able to derive a free energy estimate.

\section{Appendix E. Free-EnERGY-Dissipative discretizATION of A LiE-FORMulation}

We discuss here some discretization of the Oldroyd-B system where the equation for the stress tensor is reformulated using a Lie derivative along the deformation gradient (see Rem. 5.5 and [32]). We want to show that some discretizations of the Lie-formulation could also satisfy a discrete free energy inequality. 
TABLE 3. Summary of projected terms in the Navier-Stokes (NS) and Oldroyd-B (OB) equations for $\boldsymbol{\sigma}_{h} / \boldsymbol{\psi}_{h}$ in $\left(\mathbb{P}_{1, \mathrm{disc}}\right)^{\frac{d(d+1)}{2}}$.

\begin{tabular}{|c|c|c|}
\hline$\nabla u \ldots$ & $\boldsymbol{\sigma}_{h} \in\left(\mathbb{P}_{1, \mathrm{disc}}\right)^{\frac{d(d+1)}{2}}$ & $\boldsymbol{\psi}_{h} \in\left(\mathbb{P}_{1, \mathrm{disc}}\right)^{\frac{d(d+1)}{2}}$ \\
\hline $\operatorname{In}\left(\mathbb{P}_{0}\right)^{d^{2}}$ & $\begin{array}{l}\pi_{h}\left(\boldsymbol{\sigma}_{h}^{n}\right) \text { in time derivative } \\
\text { (incl. flux term in DG) }\end{array}$ & $\begin{array}{l}\pi_{h}\left(\boldsymbol{\psi}_{h}^{n}\right) \text { in time derivative } \\
\text { (incl. flux term in DG) } \\
+ \text { implicit source term } \pi_{h}\left(\mathrm{e}^{-\psi_{h}^{n+1}}\right) \text { in OB } \\
+ \text { implicit coupling term } \pi_{h}\left(\mathrm{e}^{\boldsymbol{\psi}_{h}^{n+1}}\right) \text { in NS }\end{array}$ \\
\hline Not in $\left(\mathbb{P}_{0}\right)^{d^{2}}$ & $\begin{array}{l}\pi_{h}\left(\boldsymbol{\sigma}_{h}^{n}\right) \text { in time derivative } \\
\text { (incl. flux term in DG) } \\
+ \text { implicit coupling terms } \\
\left(\pi_{h}\left(\boldsymbol{\sigma}_{h}^{n+1}\right) \text { in NS, OB) }\right.\end{array}$ & $\begin{array}{l}\pi_{h}\left(\boldsymbol{\psi}_{h}^{n}\right) \text { in time derivative } \\
\text { (incl. flux term in DG) } \\
+ \text { implicit source term } \pi_{h}\left(\mathrm{e}^{-\psi_{h}^{n+1}}\right) \text { in OB } \\
+ \text { implicit coupling terms } \\
\left(\pi_{h}\left(\mathrm{e}_{h}^{n+1}\right) \text { in NS, } \pi_{h}\left(\boldsymbol{\psi}_{h}^{n+1}\right) \text { in OB) }\right.\end{array}$ \\
\hline
\end{tabular}

Using Scott-Vogelius elements for $\left(\boldsymbol{u}_{h}, p_{h}\right)$ and piecewise constant approximations for $\boldsymbol{\sigma}_{h}$, one possible (loworder) discretization of a Lie-formulation from [32] writes:

$$
\begin{aligned}
0= & \int_{\mathcal{D}} \operatorname{Re}\left(\frac{\boldsymbol{u}_{h}^{n+1}-\boldsymbol{u}_{h}^{n}}{\Delta t}+\boldsymbol{u}_{h}^{n} \cdot \boldsymbol{\nabla} \boldsymbol{u}_{h}^{n+1}\right) \cdot \boldsymbol{v}-p_{h}^{n+1} \operatorname{div} \boldsymbol{v}+q \operatorname{div} \boldsymbol{u}_{h}^{n+1}+(1-\varepsilon) \boldsymbol{\nabla} \boldsymbol{u}_{h}^{n+1}: \boldsymbol{\nabla} \boldsymbol{v}+\frac{\varepsilon}{\mathrm{Wi}} \boldsymbol{\sigma}_{h}^{n+1}: \boldsymbol{\nabla} \boldsymbol{v} \\
& +\left(\frac{\boldsymbol{\sigma}_{h}^{n+1}-\left(\boldsymbol{I}-\Delta t \pi_{h}\left(\boldsymbol{\nabla} \boldsymbol{u}_{h}^{n+1}\right)\right)^{-1}\left(\boldsymbol{\sigma}_{h}^{n} \circ X^{n}\left(t^{n}\right)\right)\left(\boldsymbol{I}-\Delta t \pi_{h}\left(\boldsymbol{\nabla} \boldsymbol{u}_{h}^{n+1}\right)\right)^{-T}}{\Delta t}\right): \boldsymbol{\phi}+\frac{1}{\mathrm{Wi}}\left(\boldsymbol{\sigma}_{h}^{n+1}-\boldsymbol{I}\right): \boldsymbol{\phi},
\end{aligned}
$$

where the characteristic flow $X^{n}(t)$ is defined like in (3.7). The system (E.1) admits a solution such that $\left(\boldsymbol{I}-\Delta t \pi_{h}\left(\boldsymbol{\nabla} \boldsymbol{u}_{h}^{n+1}\right)\right)^{-1}$ is well-defined, provided $\Delta t$ is sufficiently small (but possible very small when $\left\|\boldsymbol{\nabla} \boldsymbol{u}_{h}^{n+1}\right\|$ is large). Besides, taking $\phi$ as the characteristic function of some element $K_{k}$, we have the following equality inside $K_{k}$ :

$$
\left(1+\frac{\Delta t}{\mathrm{Wi}}\right) \boldsymbol{\sigma}_{h}^{n+1}=\left(\boldsymbol{I}-\Delta t \pi_{h}\left(\boldsymbol{\nabla} \boldsymbol{u}_{h}^{n+1}\right)\right)^{-1}\left(\boldsymbol{\sigma}_{h}^{n} \circ X^{n}\left(t^{n}\right)\right)\left(\boldsymbol{I}-\Delta t \pi_{h}\left(\nabla \boldsymbol{u}_{h}^{n+1}\right)\right)^{-T}+\frac{\Delta t}{\mathrm{Wi}} \boldsymbol{I} .
$$

Then it is clear that the system (E.1) preserves the non-negativity of $\boldsymbol{\sigma}_{h}^{n}$. Moreover, it is possible to derive the free energy estimate (4.2) for the system (E.1). It suffices to take as a test function for the stress:

$$
\phi=\frac{\varepsilon}{2 \mathrm{Wi}}\left(\boldsymbol{I}-\Delta t \pi_{h}\left(\boldsymbol{\nabla} \boldsymbol{u}_{h}^{n+1}\right)\right)^{T}\left(\boldsymbol{I}-\left(\boldsymbol{\sigma}_{h}^{n+1}\right)^{-1}\right)\left(\boldsymbol{I}-\Delta t \pi_{h}\left(\boldsymbol{\nabla} \boldsymbol{u}_{h}^{n+1}\right)\right),
$$

and to proceed to the derivation of a free energy estimate using both ideas of the present work and of the work [32], after noting that:

$$
\operatorname{tr}\left(\pi_{h}\left(\boldsymbol{\nabla} \boldsymbol{u}_{h}^{n+1}\right)^{T}\left(\boldsymbol{I}-\left(\boldsymbol{\sigma}_{h}^{n+1}\right)^{-1}\right) \pi_{h}\left(\boldsymbol{\nabla} \boldsymbol{u}_{h}^{n+1}\right)\left(\left(1+\frac{\Delta t}{\mathrm{Wi}}\right) \boldsymbol{\sigma}_{h}^{n+1}-\frac{\Delta t}{\mathrm{Wi}} \boldsymbol{I}\right)\right) \geq 0
$$


the proof of which is completely similar to the proof of (1.9), using the fact that $\left(1+\frac{\Delta t}{W_{i}}\right) \sigma_{h}^{n+1}-\frac{\Delta t}{W i} \boldsymbol{I}$ is symmetric positive definite (provided $\Delta t$ is sufficiently small) and $\pi_{h}\left(\boldsymbol{\nabla} \boldsymbol{u}_{h}^{n+1}\right)^{T}\left(\boldsymbol{I}-\left(\boldsymbol{\sigma}_{h}^{n+1}\right)^{-1}\right) \pi_{h}\left(\boldsymbol{\nabla} \boldsymbol{u}_{h}^{n+1}\right)$ is symmetric positive semi-definite.

Acknowledgements. We acknowledge financial support from the France Israel Teamwork in Sciences. We would also like to thank A. Ern and J.W. Barrett for fruitful discussions, and the anonymous referees for their careful reading of the manuscript.

\section{REFERENCES}

[1] D.N. Arnold and J. Qin, Quadratic velocity/linear pressure Stokes elements, in Advances in Computer Methods for Partial Differential Equations, Volume VII, R. Vichnevetsky and R.S. Steplemen Eds. (1992).

[2] M. Bajaj, M. Pasquali and J.R. Prakash, Coil-stretch transition and the breakdown of computations for viscoelastic fluid flow around a confined cylinder. J. Rheol. 52 (2008) 197-223.

[3] J. Baranger and A. Machmoum, Existence of approximate solutions and error bounds for viscoelastic fluid flow: Characteristics method. Comput. Methods Appl. Mech. Engrg. 148 (1997) 39-52.

[4] J.W. Barrett and S. Boyaval, Convergence of a finite element approximation to a regularized Oldroyd-B model (in preparation).

[5] J.W. Barrett, C. Schwab and E. Süli, Existence of global weak solutions for some polymeric flow models. Math. Mod. Meth. Appl. Sci. 15 (2005) 939-983.

[6] A.N. Beris and B.J. Edwards, Thermodynamics of flowing systems with internal microstructure. Oxford University Press (1994).

[7] J. Bonvin, M. Picasso and R. Stenberg, GLS and EVSS methods for a three fields Stokes problem arising from viscoelastic flows. Comp. Meth. Appl. Mech. Eng. 190 (2001) 3893-3914.

[8] F. Brezzi and J. Pitkäranta, On the stabilization of finite element approximations of the Stokes equations, in Efficient Solution of Elliptic System, W. Hackbusch Ed. (1984) 11-19.

[9] F. Brezzi, J. Douglas, Jr. and L.D. Marini, Two families of mixed finite elements for second order elliptic problems. Numer. Math. 47 (1985) 217-235.

[10] F. Brezzi, J. Douglas, Jr. and L.D. Marini, Recent results on mixed finite element methods for second order elliptic problems, in Vistas in Applied Mathematics: Numerical Analysis, Atmospheric Sciences, Immunology, A.V. Balakrishnan, A.A. Dorodnitsyn and J.L. Lions Eds. (1986) 25-43.

[11] R. Codina, Comparison of some finite element methods for solving the diffusion-convection-reaction equation. Comp. Meth. Appl. Mech. Engrg. 156 (1998) 185-210.

[12] M. Crouzeix and P.A. Raviart, Conforming and non-conforming finite element methods for solving the stationary Stokes equations. RAIRO Anal. Numér. 3 (1973) 33-75.

[13] A. Ern and J.-L. Guermond, Theory and practice of finite elements. Springer Verlag, New-York (2004).

[14] R. Fattal and R. Kupferman, Constitutive laws for the matrix-logarithm of the conformation tensor. J. Non-Newtonian Fluid Mech. 123 (2004) 281-285.

[15] R. Fattal and R. Kupferman, Time-dependent simulation of viscoelastic flows at high Weissenberg number using the logconformation representation. J. Non-Newtonian Fluid Mech. 126 (2005) 23-37.

[16] A. Fattal, O.H. Hald, G. Katriel and R. Kupferman, Global stability of equilibrium manifolds, and "peaking" behavior in quadratic differential systems related to viscoelastic models. J. Non-Newtonian Fluid Mech. 144 (2007) 30-41.

[17] E. Fernández-Cara, F. Guillén and R.R. Ortega, Mathematical modeling and analysis of viscoelastic fluids of the Oldroyd kind, in Handbook of Numerical Analysis, Vol. 8, P.G. Ciarlet et al. Eds., Elsevier (2002) 543-661.

[18] J.-L. Guermond, Stabilization of Galerkin approximations of transport equations by subgrid modeling. ESAIM: M2AN 33 (1999) 1293-1316.

[19] C. Guillopé and J.C. Saut, Existence results for the flow of viscoelastic fluids with a differential constitutive law. Nonlin. Anal. TMA 15 (1990) 849-869.

[20] D. Hu and T. Lelièvre, New entropy estimates for the Oldroyd-B model, and related models. Commun. Math. Sci. 5 (2007) 906-916.

[21] T.J.R. Hughes and L.P. Franca, A new finite element formulation for CFD: VII the Stokes problem with various well-posed boundary conditions: Symmetric formulations that converge for all velocity/pressure spaces. Comp. Meth. App. Mech. Eng. 65 (1987) 85-96.

[22] M.A. Hulsen, A sufficient condition for a positive definite configuration tensor in differential models. J. Non-Newtonian Fluid Mech. 38 (1990) 93-100.

[23] M.A. Hulsen, R. Fattal and R. Kupferman, Flow of viscoelastic fluids past a cylinder at high Weissenberg number: stabilized simulations using matrix logarithms. J. Non-Newtonian Fluid Mech. 127 (2005) 27-39. 
[24] B. Jourdain, C. Le Bris, T. Lelièvre and F. Otto, Long-time asymptotics of a multiscale model for polymeric fluid flows. Arch. Ration. Mech. Anal. 181 (2006) 97-148.

[25] N. Kechkar and D. Silvester, Analysis of locally stabilized mixed finite element methods for the Stokes problem. Math. Comput. 58 (1992) $1-10$.

[26] R.A. Keiller, Numerical instability of time-dependent flows. J. Non-Newtonian Fluid Mech. 43 (1992) 229-246.

[27] R. Keunings, Simulation of viscoelastic fluid flow, in Fundamentals of Computer Modeling for Polymer Processing, C. Tucker Ed., Hanse (1989) 402-470.

[28] R. Keunings, A survey of computational rheology, in Proc. 13th Int. Congr. on Rheology, D.M. Binding et al Eds., British Society of Rheology (2000) 7-14.

[29] R. Kupferman, C. Mangoubi and E. Titi, A Beale-Kato-Majda breakdown criterion for an Oldroyd-B fluid in the creeping flow regime. Comm. Math. Sci. 6 (2008) 235-256.

[30] Y. Kwon, Finite element analysis of planar 4:1 contraction flow with the tensor-logarithmic formulation of differential constitutive equations. Korea-Australia Rheology Journal 16 (2004) 183-191.

[31] Y. Kwon and A.V. Leonov, Stability constraints in the formulation of viscoelastic constitutive equations. J. Non-Newtonian Fluid Mech. 58 (1995) 25-46.

[32] Y. Lee and J. Xu, New formulations positivity preserving discretizations and stability analysis for non-Newtonian flow models. Comput. Methods Appl. Mech. Engrg. 195 (2006) 1180-1206.

[33] A.I. Leonov, Analysis of simple constitutive equations for viscoelastic liquids. J. Non-Newton. Fluid Mech. 42 (1992) 323-350.

[34] F.-H. Lin, C. Liu and P.W. Zhang, On hydrodynamics of viscoelastic fluids. Comm. Pure Appl. Math. 58 (2005) $1437-1471$.

[35] P.L. Lions and N. Masmoudi, Global solutions for some Oldroyd models of non-Newtonian flows. Chin. Ann. Math., Ser. B 21 (2000) 131-146.

[36] A. Lozinski and R.G. Owens, An energy estimate for the Oldroyd-B model: theory and applications. J. Non-Newtonian Fluid Mech. 112 (2003) 161-176.

[37] R. Mneimne and F. Testard, Introduction à la théorie des groupes de Lie classiques. Hermann (1986).

[38] K.W. Morton, A. Priestley and E. Süli, Convergence analysis of the Lagrange-Galerkin method with non-exact integration. RAIRO Modél. Math. Anal. Numér. 22 (1988) 625-653.

[39] H.C. Öttinger, Beyond Equilibrium Thermodynamics. Wiley (2005).

[40] O. Pironneau, On the transport-diffusion algorithm and its application to the Navier-Stokes equations. Numer. Math. 3 (1982) 309-332.

[41] J.M. Rallison and E.J. Hinch, Do we understand the physics in the constitutive equation? J. Non-Newtonian Fluid Mech. 29 (1988) $37-55$.

[42] D. Sandri, Non integrable extra stress tensor solution for a flow in a bounded domain of an Oldroyd fluid. Acta Mech. 135 (1999) 95-99.

[43] L.R. Scott and M. Vogelius, Norm estimates for a maximal right inverse of the divergence operator in spaces of piecewise polynomials. RAIRO Modél. Math. Anal. Numér. 19 (1985) 111-143.

[44] E. Süli, Convergence and nonlinear stability of the Lagrange-Galerkin method for the Navier-Stokes equations. Numer. Math. 53 (1988) 459-483.

[45] R. Temam, Sur l'approximation des équations de Navier-Stokes. C. R. Acad. Sci. Paris, Sér. A 262 (1966) $219-221$.

[46] B. Thomases and M. Shelley, Emergence of singular structures in Oldroyd-B fluids. Phys. Fluids 19 (2007) 103103.

[47] P. Wapperom and M.A. Hulsen, Thermodynamics of viscoelastic fluids: the temperature equation. J. Rheol. 42 (1998) 999-1019.

[48] P. Wapperom, R. Keunings and V. Legat, The backward-tracking lagrangian particle method for transient viscoelastic flows. J. Non-Newtonian Fluid Mech. 91 (2000) 273-295. 